\title{
Dynamic Hedging Strategies Based on Changing the Pricing Parameters for Compound Ratchets
}

Samia El Khoury

\author{
A Thesis \\ In \\ The Department \\ of \\ Mathematics and Statistics
}

Presented in Partial Fulfilment of the Requirements for the degree of Master of Science (Mathematics) at Concordia University Montreal, Quebec, Canada

July, 2016

(C) Samia El Khoury, 2016 


\section{CONCORDIA UNIVERSITY \\ School of Graduate Studies}

This is to certify that the thesis prepared

By: $\quad$ Samia El Khoury

Entitled: Dynamic Hedging Strategies Based on Changing the Pricing Parameters for Compound Ratchets

and submitted in partial fulfillment of the requirements for the degree of

\section{Master of Science (Mathematics)}

complies with the regulations of the University and meets the accepted standards with respect to originality and quality.

Signed by the final examining committee:

Examiner

Dr. José Garrido

Examiner

Dr. Mélina Mailhot

Thesis Supervisor

Dr. Patrice Gaillardetz

Approved by

Chair of Department or Graduate Program Director

Dean of Faculty

Date 


\begin{abstract}
Dynamic Hedging Strategies Based on Changing the Pricing Parameters for Compound Ratchets

Samia El Khoury
\end{abstract}

Equity-Indexed Annuity products (EIAs) are becoming increasingly popular as they are tax-deferred accumulation vehicles that offer participation in the equity market growth while keeping the initial capital protected. This thesis focuses in particular on a special type of EIAs; the Compound Ratchet (CR). Sellers of this product, such as insurance companies and banks, retain the right to change one of the pricing parameters on each contract anniversary date, while promising not to cross a certain predetermined threshold. Changing these parameters can sometimes have an impact on the value of the EIA, which makes them interesting to study, especially when the issuer's changing policy is not clear. In order to reproduce the pattern of these changing parameters, a new approach of dynamically hedging the CR EIA and simultaneously protecting the issuer from hedging risk is proposed and tested.

Assuming the Black-Scholes financial framework and in the absence of mortality risk, closed-form solutions for the price and value of the CR EIA at any time throughout the contract term are obtained and then used to find the Greeks, which are in turn used build the hedging strategies. In reality, trading can only be done in discrete time, which produces hedging errors. A detailed numerical example shows that the Gammahedging strategy outperforms the Delta-hedging strategy by reducing the magnitude of these errors. However hedging risk still exists, therefore, the new approach is applied to transfer the errors from the issuer to the buyer by dynamically changing the pricing parameters. Additionally in the numerical example, the distribution of these parameters is extracted and analyzed, as well as the resulting reduction in the hedging errors, which represent the reduced cost for the issuer. 


\section{Acknowledgements}

First and foremost, I would like to express my profound gratitude to my family for their unceasing support and encouragement during my entire life. I am forever indebted to my father who taught me the value of an education, my mother who taught me the value of hard work and perseverance, and my brother who encouraged my curiosity and critical thinking. In thanking them, I would like to dedicate this thesis to my father, Professor Fadel El Khoury, who is unfortunately no longer with us to celebrate my success.

My heartfelt gratitude goes to my very supportive supervisor, Professor Patrice Gaillardetz. His expertise, patience, and insightful guidance made this work possible and added considerably to my graduate experience.

Besides my supervisor, I would like to thank the rest of my thesis committee; Professor Garrido and Professor Mailhot, for taking the time to read and provide the necessary valuable feedback.

I would like to also take this opportunity to express a deep gratitude to all of the Mathematics and Statistics department faculty members at Concordia University for their help and advisement.

Last but not the least; my sincere thanks goes to all my friends who bared a listening ear and provided continuous support throughout the entire process. 


\section{Contents}

List of Figures vii

List of Tables viii

1 Equity-Linked Products $\quad 10$

1.1 Annuities . . . . . . . . . . . . . . . . . . . 10

1.2 Fixed Annuities . . . . . . . . . . . . . . . . . . . . . 11

1.3 Variable Annuities . . . . . . . . . . . . . . . . . . . . 11

1.3.1 Investment Guarantees . . . . . . . . . . . . . . . . . . . . . 13

1.4 Equity-Indexed Annuities . . . . . . . . . . . . . . . 15

1.4.1 Point-to-Point Class of EIA . . . . . . . . . . . . . . 18

1.4 .2 Ratchet Class of EIA . . . . . . . . . . . . . . . . 20

2 The Black and Scholes Model $\quad 24$

2.1 Introduction . . . . . . . . . . . . . . . . . . . 24

2.2 The Black-Scholes Economy . . . . . . . . . . . . . . 25

2.2.1 Continuous Stochastic Processes . . . . . . . . . . . . . 26

2.2.2 Martingales . . . . . . . . . . . . . . . . . 26

2.2.3 The Martingale Valuation Principle . . . . . . . . . . . . . . 28

2.3 The Model's Assumptions . . . . . . . . . . . . . . . . . . . . . 30

2.4 The Stock Price Process . . . . . . . . . . . . . . . . . . . . . 32

2.4.1 Standard Brownian Motion Process . . . . . . . . . . . . . . . . 32

2.4.2 Generalized Brownian Motion Process . . . . . . . . . . . . . 35

2.4.3 The Stock Price as a Geometric Brownian Motion Process . . . . . 36 
2.4.4 The Money Market Account . . . . . . . . . . . . . . . . . . . 37

2.5 Pricing European Call Options in BS . . . . . . . . . . . . . . . . 38

2.5.1 Pricing Under the Martingale Valuation Principal . . . . . . . . . . 39

2.5.2 Pricing Under the Replicating Portfolio Method . . . . . . . . . . . 41

3 The Hedging Strategy $\quad 42$

3.1 Introduction . . . . . . . . . . . . . . . . . . . . . . 42

3.2 The Greeks . . . . . . . . . . . . . . . . . . . . . . . 45

3.2.1 Delta-Hedging . . . . . . . . . . . . . . . . . . 45

3.2.2 Gamma-Hedging . . . . . . . . . . . . . . . . . . . . . . . 49

3.3 Hedging in Discrete Time . . . . . . . . . . . . . . 54

3.3.1 Hedging Frequency . . . . . . . . . . . . . . . . . . . . 54

3.3 .2 Hedging Errors . . . . . . . . . . . . . . . . . 55

3.3.3 Discretization of the Stock Price Process . . . . . . . . . . . . . 57

4 Pricing Equity-Indexed Annuities $\quad 59$

4.1 Time-0 Price of Annual Compound Ratchet EIA . . . . . . . . . . . . . . . 59

4.2 Time- $t$ Price of Annual Compound Ratchet EIA . . . . . . . . . . . . . . . 66

5 Hedging Equity-Indexed Annuities $\quad 72$

5.1 Hedging of Compound Annual Ratchet EIA . . . . . . . . . . . . . . 72

5.1 .1 Delta-hedging strategy . . . . . . . . . . . . . . 73

5.1 .2 Delta-hedging Errors . . . . . . . . . . . . . . . 76

5.1.3 Gamma-hedging Strategy . . . . . . . . . . . . . . . 79

5.1 .4 Gamma-hedging Errors . . . . . . . . . . . . . . . 82

5.2 Dynamic Risk Management Strategy . . . . . . . . . . . . . . . . . . 85

$5.2 .1 \quad$ Numerical Analysis . . . . . . . . . . . . . . . . . . . . . . . 88

5.2.2 Hedging Errors After Applying the Proposed Strategy . . . . . . . . 101 


\section{List of Figures}

2.1 Three samples paths of standard Brownian motion processes. . . . . . . . . 34

5.1 Present value of Delta-hedging errors. . . . . . . . . . . . . . 78

5.2 Present value of Gamma-hedging errors. . . . . . . . . . . . . . . 84

5.3 Time-0 value of an Annual Compound Ratchet EIA as a function of $c_{0}$. . 89

5.4 Histogram of $c$ at the default parameter set. . . . . . . . . . . . . . 90

5.5 Effects of changing the number of trading dates $m \ldots \ldots$. . . . . . . 92

5.6 Effects of changing the contract term $T \ldots \ldots \ldots \ldots$

5.7 Effects of changing the participation rate $\alpha . \ldots \ldots . \ldots . \ldots$

5.8 Time-0 value of an Annual Compound Ratchet EIA as a function of $\alpha_{0}$. . . 99

5.9 Histograms comparing the distribution of $\alpha$ as the number of trading dates per year increases. . . . . . . . . . . . . . . . . . . 100

5.10 Cost of hedge after applying the proposed method, if the $5 \%$ quantile is considered as the minimum rate. . . . . . . . . . . . . . 103 


\section{List of Tables}

5.1 Quantiles of the distribution of $T H E_{0}^{\Delta}$, in percentages. . . . . . . . . . 79

5.2 Quantiles of the distribution of $c$, in percentages. . . . . . . . . . 91

5.3 Effects of changing the number of trading dates $m$ (in percentages). . . . . 91

5.4 Effects of changing the contract term $T$ (in percentages) . . . . . . . . . 93

5.5 Effects of changing the floor rate $f$ (in percentages) . . . . . . . . . . 94

5.6 Effects of changing the participation rate $\alpha$ (in percentages) . . . . . . 96

5.7 Values of the $5 \%$ quantile (in percentages) for two values of $\sigma$ and $r$ as $\alpha$ increases. . . . . . . . . . . . . . . . . . 96

5.8 Magnitude of increment in the $5 \%$ quantile of $c$ as $r$ increases from $3 \%$ to

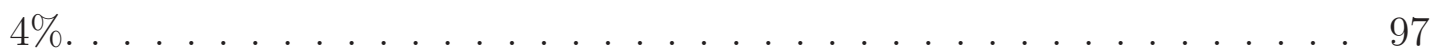

5.9 Magnitude of decrement in the $5 \%$ quantile of $c$ as $\sigma$ increases from $20 \%$ to $30 \%$. . . . . . . . . . . . . . . . . . 97

5.10 Values of the $5 \%$ quantile (in percentages) showing the effect of $\alpha$ in low and high volatility markets. . . . . . . . . . . . . . . . 97

5.11 Quantiles of the distribution of $\alpha$, in percentages. . . . . . . . . . 101

5.12 Mean, standard deviation, and $1 \%$ quantile of the final hedging cost for different choices of minimum rates. . . . . . . . . . . . . . . . 102 


\section{Introduction}

"If the stock market goes up, you win; if the stock market drops, you win even more because you don't lose." says an insurance salesman while marketing an Equity-Indexed Annuity (EIA) product. Known as segregated funds in Canada, EIA products are one of the most popular financial derivatives that combine three important main features; capital preservation, participation in the upturns of equity markets, and a tax-deferred accumulation vehicle. They were first introduced in 1995 by Keyport Life Insurance Co. Nowadays, the US's top largest Indexed-Annuity seller is Allianz Life Insurance Co. of North America. According to Todd Giesing, assistant research director at LIMRA Secure Retirement Research, "sales of EIAs have experienced 8 consecutive years of positive growth" to hit a record of 54.5 billion in 2015 (see LIMRA (2015)). In the light of the growing importance of Equity Indexed Annuities, they have received great attention in the academic literature, from product description, valuation, and hedging.

For instance, different classes and designs of EIAs are available in the marketplace, offering an extensive variety of features and crediting methods that define their payoffs structure. In particular, Annual Ratchet EIAs have accounted for slightly more than $94 \%$ of EIA sales volume in the third quarter of year 2005, with the remaining $6 \%$ of sales coming from the Point-to-Point EIA class products (see Marrion (2005)). So far, the Annual Ratchet or Reset - also known as Cliquet in French - remains the most popular class of EIA, mainly for its appealing yearly interest "lock-in" feature, along with the "reset" feature where the index level used to determine the index-linked gain is reset to its current value at the beginning of each year (i.e. on the policy anniversary date). In addition, 
this reset feature also applies on the annual participation and cap rates, whichever are included in the design. That is, issuers of nearly all Annual Ratchet products nowadays retain the right to reset (or change) either of these rates on a yearly basis throughout the term of the contract, yet with a promise of not going below a minimum rate agreed upon at inception. Consequently, this thesis makes use of the this special Ratchet feature of EIAs to protect issuers from hedging risk.

The two papers by Brennan and Schwartz (1976) and Boyle and Schwartz (1977) have laid the foundation of the research work on equity-linked life insurance contracts, by extending the Black-Scholes no-arbitrage pricing framework to the case of insurance contracts, which led to the development of many extensions to price the different types of Variable Annuities and other Equity-linked products.

Additionally, Boyle et al. (2001) make use of a particular type of lattice rules, known as good lattice points, to price High-Watermark EIAs - also known as lookback designs and other financial derivatives. They show that their proposed method outperforms the numerical efficiency of other suggested competitive methods for this type of EIA contracts.

Lin et al. (2009) study the pricing of Compound Ratchet and simple Point-to-Point EIAs with and without mortality risk under a Markovian regime switching model, where the dynamics of the underlying asset follow a Geometric Brownian Motion model with regime switching. The use of a regime switching model implicitly implies that the market is incomplete, thus they make use of the Esscher Transform method presented in Gerber and Shiu (1994) to determine an equivalent martingale measure for fair option valuation in incomplete markets. They also study the critical (or fair) guarantee charge embedded in variable annuities with either or both the Guaranteed Minimum Death Benefit (GMDB) and Guaranteed Minimum Maturity Benefit (GMMB) options to examine their costs. Similarly, Lin (2010) make use of the Regime Switching Jump Model (RSJM) to model the underlying asset's return in pricing Compound Ratchet and Point-to-Point EIAs with 
Term-End design, where the dynamics of the stock price process follow the risk-neutral Esscher measure. It is also noted that the Black-Scholes model can be derived from the RSJM by setting some parameters equal to zero. One of the motivations behind choosing the RSJM is that it reflects the leptokurtic feature and volatility smile of the stock price process, as well as the phenomenon of volatility clustering, which is not captured by the Black-Scholes model.

The effects of considering mortality risks in pricing EIA contracts is studied in several papers. The most well renowned ones include but are not limited to: Boyle and Schwartz (1977), Lin and Tan (2003), Gaillardetz and Lin (2006), Lin et al. (2009), Quian et al. (2010), etc... In particular, Gaillardetz and Lin (2006) proposed a market consistent valuation technique for Point-to-Point contracts with Term-End Point and High-watermark designs as well as Compound Resets with embedded annual yield spread. They start by deriving age-dependent and mortality risk adjusted martingale probability measures that reproduce the premiums of each of the three main insurance products: term-life insurance, pure endowment insurance, and endowment insurance. They also assume that the underlying index is governed by the modified Cox, Ross, and Rubenstein (1979) binomial model. Then the dependency between the financial market and the insurance market is reflected using different types of copulas.

Assuming the Black-Scholes framework, Tiong (2001) derives closed-form formulas for the prices of Point-to-Point EIAs with Term-End and High Watermark designs as well as Compound Ratchet EIAs using Esscher Transform. Nevertheless, in all three cases, Tiong (2001) modifies the payoffs such that the participation rate is applied on the natural logarithm of the index-linked gain. However, Hardy (2004) argues that the pricing results for Point-to-Point and Ratchet EIAs are somewhat similar to those derived using the traditional risk-neutral expectations of the discounted payoff under the $\mathbb{Q}$-measure in the Black-Scholes framework.

Another variation on the crediting method of EIAs can be found in Lee (2003). With 
the purpose of increasing the participation rate $\alpha$, he applies up-and-in barrier options on Point-to-Point and Compound Ratchet EIAs, and price them using Esscher Transforms under Black-Scholes framework. The up-and-in barrier feature makes the payoff path-dependent by adding the condition that the stock price rises above a certain predetermined threshold. Additionally, he studies the pricing of partial-time look-back EIAs with and without variable guarantees based on the method of Heynen and Kat (1994).

Another interesting paper that outlines a different aspect of the financial market is Lin and Tan (2003). They assume that both the underlying index and interest rates are stochastic and model them jointly by using the Vasicek model for the term structure of interest rates. The reason why stochastic interest rates are crucial in pricing EIAs is because of the embedded guarantees associated with most contracts whose maturities range up to ten years. Thus, they state that it is unreasonable to assume constant interest rates for such a long duration. However, by incorporating mortality risk and stochastic interest rates they lose the tractability of pricing and hedging Compound Ratchet EIAs, and therefore resort to simulations. Similarly, Kijima and Wong (2007) assume that the risk-free interest rate is stochastic and follows the extended Vasicek model, and they price the Ratchet EIA under the arbitrage-free pricing principle. For a comprehensive discussion and analysis of different stochastic models for the term structure of interest rates and their importance in option valuation we refer the reader to Schulmerich (2010).

Hardy (2004) gives an explicit pricing formula for the Compound Ratchet EIA under the Black-Scholes market assumptions using a risk-neutral valuation. She argues that the sum of log-normal random variables in the complex design of the Simple Ratchet payoff with the embedded floor and cap rates makes it impossible to find a closed-form solution to price such contracts. Therefore, most researchers and practitioners refer to numerical methods to solve this problem. The most common approach is using Monte Carlo simulations. This is done by first generating paths of the underlying stock price process $S_{t}$ under the risk-neutral measure $\mathbb{Q}$. Then the price is estimated by taking the average of 
each path's payoff discounted back to time zero by the risk-free rate of return $r$. Several other pricing methods are also presented in the literature. For instance, Hardy (2004) proposes a non-recombining tri-nomial lattice method to value Simple Ratchet EIAs, and shows that the valuation results are significantly better and the method more efficient than standard Monte Carlo Simulations. However, Hsieh and Chiu (2007) under the same market assumptions, derive a closed-form solution for pricing Simple Ratchet EIA contracts, somewhat in the same manner as Hardy (2004) derived the Compound Ratchet pricing formula.

Additionally, as explained by several works, including Hardy (2003) and Hsieh and Chiu (2007), the minimum accumulated guarantee on the initial investment over the entire term of the EIA contract (or global floor) is not analytically tractable, and thus no closed-form solutions can be obtained to price such Ratchet EIA contract neither in the case of the Compound Ratchet nor in the case of the Simple Ratchet EIA. With this in mind, the most often used approach to price such contracts is through Monte Carlo simulation. Variance reduction techniques are often used to improve the accuracy of the point estimates. In particular, Hardy (2004) proposes using the payoff of the Compound Ratchet as a control variate to price the same contract with the embedded minimum term guarantee. Similarly, Hsieh and Chiu (2007) suggest using the payoff for the Simple Ratchet, after deriving the closed-form pricing formula, to price the contract with the embedded minimum term guarantee. Moreover, Hardy (2004) shows that the added price of the minimum term guarantee is small relative to that of the EIA in general and is not sensitive to the type of the Ratchet, nor to the cap and participation rates. Therefore, this minimum "life of contract guarantee" will not be considered in this thesis.

The recent economical crisis had shed light upon the importance of hedging. Before this massive event, many insurers used to mainly rely on re-insuring their future liabilities. Facing the crisis, the major fall in equity prices also affected re-insurance companies, which pushed insurers to put more efforts on developing self-hedging strategies 
that would protect them from market downturns. It is estimated that companies that adopted successful hedging strategies saved the industry around $\$ 40$ billion by late 2008 (see McKinsey and Company (2009)).

Various hedging strategies are suggested in the literature. Some study the application of dynamic risk measures on hedging Equity-Linked products. In particular, Hardy and Wirch (2004) use the Iterated Conditional Tail Expectation risk measure (ICTE) to hedge against the risk of additional capital by reducing the cash flow volatility. Similarly, Moghtadai (2014) studies the application of the Iterated Conditional Value at Risk $(I C V a R)$ in minimizing the cost of the hedging portfolio with the constraint on the risk measure being non-positive.

Dynamic hedging techniques using the replicating portfolio of stocks and money market accounts are often used in the academic literature for hedging Equity-Linked products. For instance, Moller (1998, 2001a) determine risk-minimization hedging strategies for Equity-Linked life insurance products under a generalized Black-Scholes framework, where the financial market consists of only stocks and money market accounts in a continuoustime setting. Whereas Moller (2001b) presents a similar work in a discrete-time setting under the Cox, Ross, and Rubinstein (1979) model. Other economic models are also suggested, for example Jaimungal (2004) uses the Greeks: Delta, Gamma and Vega to dynamically hedge Ratchet EIAs in a Variance-Gamma economy, and analyzes the results by comparing them to those obtained in the Black-Scholes and Heston model such as the work done in MacKay (2011).

In addition to the normal dynamic hedging techniques, Bernard and Boyle (2011) propose a complementary technique called natural hedge that protects issuers against market volatility risk by building a portfolio of policies with different payoffs. In particular, they study how the interactions of two different EIA designs: the simple Point-to-Point and the 
Monthly Sum Cap ${ }^{1}$ EIA, with different exposure to volatility combined in one portfolio can be useful in stabilizing the market value of their liabilities and thus reducing the volatility.

With all that being said, this thesis focuses on the Annual Compound Ratchet EIA with Term-End Point design. By assuming that mortality risk can be easily diversified through pooling, the valuation of such an insurance product becomes similar to any pure financial derivative security. Following Hardy (2004), the valuation of this product is carried out assuming the Black-Scholes model. Adopting this financial framework comes with several implications. In complete markets, the payoff of any financial derivative security can be perfectly replicated by creating a replicating portfolio of risky and nonrisky assets, that can be used to price and/or hedge this security. Most importantly, the dynamics of the stock price process follow a Geometric Brownian Motion with drift. Under this model, the discounted price process of any derivative security is a martingale under the risk-neutral probability measure $\mathbb{Q}$. Therefore, a closed-form expression of the time-zero price of this EIA product is obtained, and another closed-form expression for its value at any time $t$ from inception until maturity of the contract is also obtained. These expressions are then used to find the Greeks. Representing the sensitivities of the price to certain parameters, the Greeks are used to construct the replicating portfolio, which is in turn used to develop dynamic hedging strategies.

The only time the assumption of market completeness is violated is by applying the continuous time strategies in a discrete time setting, which gives rise to hedging errors. These hedging errors represent the cost incurred by the issuer of the contract from applying the hedging strategy. The main objective of this thesis is to propose an approach that protects the issuer, as much as possible, from these dynamic hedging errors. With that same purpose, Gaillardetz and Lakhmiri (2011) set up a replicating portfolio of shares and money market accounts for equity-linked products, then they introduce a loaded contract premium using a risk measure based on the distribution of the hedging errors by chang-

\footnotetext{
${ }^{1}$ A Simple Monthly Ratchet with a local cap rate.
} 
ing the participation rate $\alpha$, either statically or dynamically. Inspired by their work, we propose a new method, complementary to the dynamic hedging strategy, that reproduces the pattern of the changing participation and cap rates, and at the same time that it dynamically eliminates the hedging errors by transferring them to the buyer. This can be done by changing the value of the EIA through resetting the pricing parameters at anniversary dates, such that this new value incorporates the amounts of yearly hedging errors. By following this method, the issuer is able to transfer nearly all the hedging errors to the buyer, except for those during the last year, which will then make up the new reduced cost of the dynamic hedging strategy.

This thesis is structured as follows: Chapter 1 presents an overview of Equity-Linked products. It gives a detailed discussion on Variable Annuities with their different embedded guarantees, and on Equity-Indexed Annuities with their various classes and designs.

Chapter 2 gives an overview of the Black-Scholes financial framework that will be considered throughout this thesis. In particular, the Martingale Valuation Principle is introduced, which will be later used to price the Compound Ratchet EIA. The model assumptions and the dynamics of the stock price process are presented, as well as the pricing of European Call options using to approaches; the martingale valuation principle and the replicating portfolio method.

Chapter 3 introduces the notion of hedging and that of the Greeks, and shows how the Greeks can be used to construct hedging strategies for any derivative security in general. The formula for pricing European Call options, introduced in Chapter 2, is used as an example to find the Greeks based on the Delta and the Gamma-hedging strategies. In reality, hedging must be done in a discrete time setting, which leads to hedging errors. Therefore, the concepts of discrete-time hedging and hedging errors are presented. Finally, to complete the discrete-time model, the discretization scheme of the stock price process is described, which will be later used to simulate stock price paths in the numerical analysis. 
Chapter 4 introduces the closed-form expressions for the time-zero price and the time- $t$ value of the Annual Compound Ratchet EIA contract. This is done using the Martingale Valuation Principle described in Chapter 2.

Finally, Chapter 5 uses the results of Chapter 4 to obtain closed-form expressions for the Greeks (Delta and Gamma) of the Annual Compound Ratchet EIA, with the purpose of applying the hedging strategies described in Chapter 3. At first, the Delta-hedging strategy is implemented in discrete time. Delta-hedging errors are extracted and used to assess the performance of this strategy. Then, with the purpose of improving the hedging strategy by reducing the magnitude of the errors, the Gamma-hedging strategy is applied and its performance is assessed through analyzing the resulting Gamma-hedging errors. Nevertheless, this improvement only reduces the magnitude of the cash flows but does not totally eliminate the risk. Therefore the new proposed hedging approach of transferring the gains and losses to the buyer by changing either the pricing parameter is implemented. A detailed numerical analysis is conducted on an Annual Compound Ratchet contract and the distribution of the pricing parameter is analyzed. Finally, the cost of the hedging strategy is reduced to the total hedging errors of the last year of the contract only, which are extracted and used to analyze the efficiency of this method. 


\section{Chapter 1}

\section{Equity-Linked Products}

\subsection{Annuities}

According to the IRI 2009 Annuity Fact Book, the first annuity was issued in year 1759 to Presbyterian ministers and their families by a Pennsylvania company. They were then offered to the general public for the first time in 1912. However, the concept of annuities goes back to the Roman times, when citizen used to buy themselves lifetime yearly payments by making one single payment to a contract named annua.

Nowadays, annuities gained many variations and became a part of a wide number of insurance and financial products. In their simplest forms, annuities are a contract between two parties; the insurer and the insured, also called the annuitant. Besides pensions and Social Securities, the main purpose of annuities is for retirement planning, for they are a money accumulation vehicle that provides the annuitant with a guaranteed future income stream. Therefore, annuities give the annuitant protection against outliving his own resources. Thus longevity risk is transfered from the annuitant to the insurance company, which manages it by risk pooling and diversification.

In general, annuities have two phases; the accumulation (or savings) phase, during which the investment is growing, and the annuitization (or payout) phase, during which the annuitant receives the income or the gain from his investment as a stream of pay- 
ments at regular intervals until a specified period has ended, or an event such as death has occurred.

\subsection{Fixed Annuities}

The simplest form of annuities is the Fixed Annuity (FA). Similar to Guaranteed Investment Certificates (GICs), these contracts are mostly bought by people who are not fully participating in the work force to help them stabilize their income. The initial amount of money invested in the FA is guaranteed to accumulate in a tax-deferred manner at a fixed pre-determined interest rate during the accumulation phase. Hence, the regular payments received by the annuitant during the annuitization period are fixed and known in advance.

Besides the benefit of tax deferral, FAs offer the advantage of being the less risky of investment options due to the guaranteed fixed return, which makes them attractive to risk-averse investors. However, one of the disadvantages of FAs is that their fixed income payments do not account for inflation over the long term, which decreases the value of the annuity and its purchasing power.

Those who are seeking more investment flexibility and more opportunity for market growth are usually more interested in another riskier type of annuities called the Variable Annuity. It is a hybrid type of investment that mixes insurance and financial securities.

\subsection{Variable Annuities}

Variable Annuities (VA) are managed fund products that were first introduced in the United States in the 1970's. So far, the U.S. has been the largest VA market in the world. Also sold in Canada and the United Kingdom, where VA contracts are known as segregated funds and unit-linked insurance products, respectively. Sloane (1970) is one of 
the earliest papers that analyzed VAs from an actuarial perspective.

Generally, VAs are purchased with a single premium amount paid up-front. Then the writer of the contract, typically insurance companies, invest this initial capital in separate accounts similar to mutual funds, called sub-accounts. These sub-accounts fall into two categories: variable and fixed. A wide range of investment options are offered, constituting a mixture of stocks, bonds, derivatives, commodities or other investments, and make up the variable sub-accounts. In addition to that, a fixed rate of return is guaranteed on the initial investment by the insurance company in the fixed sub-account. This interest rate may be reset periodically by the insurer, but he will usually provide a guaranteed minimum, (3\% for example). Most VAs include participation in both fixed and variable sub-accounts, however, some of them invest in only the variable one, like the Investment Only Variable Annuities (IOVA) for example.

Unlike Fixed Annuities, the value of the portfolio will vary depending on the investment options chosen. The allocation of the initial amount or premium into the different investment options is a choice for the investor. Hence, the buyer bears all the investment risk for the amounts allocated to the variable sub-accounts. However, when applicable, his initial investment remains protected by the guaranteed minimum interest rate, thus limiting the downside risk. Usually, a variety of portfolios are pre-built and presented to the investor by the insurance company, with different objectives and investment strategies. Thus the investor's choice relies mainly upon his risk appetite. Although the portfolio compositions are build by the insurer, their management is done outside the insurance company. In some cases, when the initial investment amount is relatively high, buyers are given the option to design and build their own portfolios.

VA contracts gained much popularity by being long term investment vehicles for retirement and pension plans with some tax advantages. During the accumulation phase of the VA, investment gains accumulate on a tax-deferred basis. Furthermore, referring to the 
fact that the payment of income taxes on interests and dividends earned is deferred until the payout phase. In addition, VAs offer tax-free transfers among different investment options. That is, the re-allocation of funds from one variable sub-account to another, or even from and into the fixed sub-account, within that same annuity is also free of taxation.

\subsubsection{Investment Guarantees}

Typically, VA policies are sold with at least one optional guarantee, referred to as the Guaranteed Minimum Benefit or GMxB. The " $\mathrm{x}$ " describes the nature of the guarantee embedded in these products, also commonly known as the "rider". These guarantees, introduced in the 1990's, fall into two main categories: the Guaranteed Minimum Death Benefits GMDB, and the Guaranteed Minimum Life Benefits GMLB.

The embedded GMDB option was introduced in 1980. At the signing of the VA contract, the policyholder assigns one or many beneficiaries, to whom the insurance company (or the writer in general) promises to return a certain amount referred to as the Death Benefit, should the policyholder die during the accumulation phase. Several methods for determining this amount exist. The earliest and simplest one is the Return of Premium Death Benefit, where the Death Benefit is the maximum of the initial amount invested by the policyholder and the market value of the account at the time of his death. A second form is the Annual Roll-Up Death Benefit, where the Death Benefit is the greater of the initial amount invested accumulated at the pre-defined fixed roll-up rate and the current account balance. Another variation is called the Annual Ratchet Death Benefit, where the Death Benefit takes the form of the maximum of the highest "anniversary" account balance and the current balance. The "anniversary" is typically every year, but it could also be monthly or every 5 years. This feature is interesting because it "locks in" the high returns at the anniversary date, and thus would be beneficial and rewarding if the investment performs very badly thereafter. In any case, the Death Benefit is adjusted for any withdrawals that might have been made. Also note that when the death benefit is 
earned by the named beneficiary, he gets taxed on it as ordinary income.

The GMLB products, in general, provide protection against investment risk or equity market declines during the accumulation phase, as well as mortality risk. They comprise three main categories, each with a different objective. The first two issued riders are the Guaranteed Minimum Income Benefit (GMIB) and the Guaranteed Minimum Accumulation Benefit (GMAB). The GMAB rider guarantees a minimum account value at maturity of the contract, that could be annuitized or paid as a lump-sum. This minimum value could be the initial investment or a roll-up benefit base which is the initial investment compounded at the constant roll-up rate. Similarly for the GMIB rider, except that it specifically requires the accumulated value to be annuitized, and thus guarantees a certain income stream (typically for lifetime) during the payout phase, regardless of the performance of the investment strategy used during the accumulation phase. In this case, the fixed annuity payments and the annuitization rates are already specified at inception of the contract.

Issued in 2002, the third type of living benefits rider is the Guaranteed Minimum Withdrawal Benefit. It provides the policyholder the possibility to withdraw a certain pre-specified amount periodically (usually each year) during the life of the contract, regardless whether the account value has fallen below this amount and even if it reaches zero. Commonly, this would happen under some limitations on the periodic amounts withdrawn, along with the condition that the sum of the total withdrawals remains less than or equal the initial capital invested. Thus, these withdrawals decrease the account value, and any remaining fund is then returned to the policyholder at maturity of the contract. Later in 2003, the U.S. market introduced a new type of riders; the Guaranteed Lifelong Withdrawal Benefit (GLWB), also known as the Guaranteed Minimum Withdrawal Benefit for Life. As its name suggests, this rider gives the annuitant the possibility to make lifetime withdrawal from his VA account. In this case, there are no conditions that limit the sum of the total withdrawals, only a maximum amount is set, since even if the account 
value drops to zero, the policyholder can still periodically withdraw the guaranteed and pre-specified amount as long as he is still alive.

Many research papers can be found in the financial and actuarial literature on the pricing and valuation of Variable Annuity products, with regards to the guaranteed embedded options in them. As previously mentioned, Brennan and Schwartz (1976) and Boyle and Schwartz (1977) have laid the foundation of pricing equity-linked life insurance contracts assuming the Black-Scholes framework, and many extensions followed. For instance, Milevsky and Posner (2001) use the risk neutral option pricing theory to value various types of GMDB options in VAs by treating them as Titanic Options. Similarly, Haberman and Piscopo (2008) use the Black-Scholes model to value the GMDB as a weighted average price of a set of deterministic put options having stochastic maturity dates, weighted by the probability of death. Krayzler et al. (2011) find explicit solutions for the price of GMABs by using the Hull-White-Black-Scholes hybrid model with timedependent volatility and stochastic mortality. Marshall et al. (2010) price GMIBs under stochastic interest rates. Papers dealing with the standard no-arbitrage pricing model for GMWB and GLWB options include respectively; Chen et al. (2008) under the assumption of optimal policyholder behavior, and Haberman and Piscopo (2011) focusing on the impact of mortality risk. In general, Bauer et al. (2008) and Bacinello et al. (2011) set a general unifying framework for consistently pricing any type of GMxB rider.

\subsection{Equity-Indexed Annuities}

The recent economical crisis has negatively affected the VA market. With the stock market volatility highly increasing and the risk free interest rates reaching their lowest, investors started drifting away from VAs, seeking safety and protection for their investments. Thus, a very special type of investment has dominated the annuity market; The Equity-Indexed Annuity.

Also known as Fixed-Indexed Insurance Products, Equity-Indexed Annuities (EIAs) 
are a type of financial instruments that is classified in between fixed annuities and variable annuities. Having their return linked to the performance of a specific stock or market index (like the S\&P 500 price index for example), EIAs offer full or partial participation in the index-linked gain, even though assets are not directly invested in the underlying equity index. Although an EIA contract resembles a VA contract with a GMxB option, their difference lies in that the net initial capital of an EIA is essentially invested in riskfree bonds. During the term of the contract, the EIA behaves closely like a FA, until the index-linked gain is credited as interest at maturity.

The gain in the index to be credited to the annuity is set by a parameter called the participation rate. This feature makes their return more variable than a fixed annuity, but less variable than a variable annuity. Also, EIA buyers benefit from a guaranteed minimum interest rate which provides a floor for the return on their investment, thus limiting the downside risk of the equity market and protecting the initial capital. In addition to these two features, most EIAs impose a cap or an upper limit on their credited return, thus limiting the gain from the index as the stock price increases. As a result, these three features combined make EIAs riskier but more profitable than fixed annuities, but less profitable with less market risk than variable annuities, which captures the interest of a big number of investors.

The typical term of an EIA contract ranges from 5 to 10 years. Seven years EIAs are the most common on the market, which makes them short-term investments compared to pure insurance contracts, but long compared to financial products, yet not as long as the twenty to thirty years VAs. Most EIAs are based on the Standard \& Poor 500 index, but other indices are also used. Some EIAs even allow investors to select one or more indices for their investment.

Since first introduced by Keyport Life Insurance Company in 1995, EIAs have gained very much popularity. Their sales have consistently increased from $\$ 5.5$ billion in year 
2000 , to reach $\$ 23.1$ billion in just four years (2004) and hit their high record of $\$ 33.9$ billion in the fourth quarter of the year 2012 when VA sales decreased by $7 \%$ and that of FAs by $11 \%$ compared to year 2011. According to Todd Giesing, assistant research director at LIMRA Secure Retirement Research, the increase in the EIA sales was led by the decrease of the VA market share. In 2015, FA sales increase to $\$ 74$ billion and EIAs continue to experience a remarkable growth and reach record-breaking levels of $\$ 38.4$ billion YTD.

EIA products may seem very attractive when only seen as popular investment vehicles offering participation in the equity market growth while keeping the initial capital investment protected. However, this simple view is somewhat misleading as the complexity of EIAs lies within the choice of the crediting method used. See Hardy (2003) for a detailed and comprehensive explanation.

Two main classes of EIAs exist on the financial market: the Point-to-Point EIA and the Ratchet EIA. Generally speaking, the difference between them lies in the crediting method applied to define the payoff of the contract, which is the way used to calculate the gain from the underlying index and adding it to the EIA contract's return as interest.

Various crediting parameters could be seen in both classes, and they include:

- The Participation Rate - denoted by $\alpha$ - is the percentage of the gain from the underlying index credited to the EIA as interest.

- The Cap Rate - denoted by $c$ - is the maximum interest rate that can be credited to the EIA, representing an upper bound for the partial (or credited) index gain.

- The Floor Rate - denoted by $f$ - is the minimum interest rate that can be credited to the EIA, representing a lower bound for the partial index gain.

- The Spread - denoted by $s$ - also known as the Margin, is the percentage by which the index's gain is reduced before being credited to the contract as interest. 
- The Roll-Up Rate - denoted by $g$ - is the guaranteed minimum interest rate on a fraction $\beta$ of the initial capital invested.

It is important to note that both the participation and cap rates can be either fixed or annually reset by the writer of the EIA contract. This is an important feature that will be used in the main work of the thesis. In addition to the spread, these three parameters reduce the market risk of the EIA by limiting its return, and thus making its payoff less variable.

\subsubsection{Point-to-Point Class of EIA}

The simplest class of EIAs is the Point-to-Point. It measures the index gain between two discrete points in time from inception $(0)$ until maturity $(T)$ of the contract. In general, the payoff of such a contract with an embedded local cap rate $c$ has the following expression:

$$
\operatorname{PTP}(T)=I C \times \min \left\{1+\alpha\left(R_{t}-1\right),(1+c)^{T}\right\}
$$

where $I C$ is the initial capital invested, and $R_{t}$ is the return on the index to be defined depending on the design.

If the EIA offers the embedded GMAB option as protection against the loss from a down market with $g \%$ global minimum annual interest rate guaranteed on the portion $\beta$ of the initial investment $I C$ over the entire term of the contract, then the payoff in (1.4.1) becomes:

$$
P T P^{A}(T)=I C \times \max \left\{\min \left\{1+\alpha\left(R_{t}-1\right),(1+c)^{T}\right\}, \beta(1+g)^{T}\right\} .
$$

Also, if the EIA includes a spread that decreases the amount of index return credited to the contract, the payoff in (1.4.1) becomes:

$$
P T P^{s}(T)=I C \times \min \left\{1+\alpha\left(R_{t}-1\right)-s,(1+c)^{T}\right\} .
$$


Under the Point-to-Point class, there are three different designs commonly used in practice; the Term-End point design, the Asian-End design, and the High-Watermark design. They differ in the crediting method used to measure the index-linked "gain" $R_{t}$.

\subsubsection{PTP with Term-End point design}

Most commonly referred to as the Simple Point-to-Point, this design calculates the growth of the index based on the difference between its value at inception and at maturity of the contract. In this case we have with $R_{t}=\frac{S(T)}{S(0)}$, and the payoff in (1.4.1) becomes:

$$
\operatorname{PTP}^{T E}(T)=I C \times \min \left\{1+\alpha\left(\frac{S(T)}{S(0)}-1\right),(1+c)^{T}\right\}
$$

\subsubsection{PTP with Asian-End design}

Similarly to an Asian option, this design calculates the index-linked gain by averaging the index values during the last year of the contract term. Assuming index values can be measured $m$ times a year, we have with $R_{t}=\frac{\sum_{t=1}^{m} S\left(T-1+\frac{t}{m}\right)}{m S(T-1)}$, and (1.4.1) becomes:

$$
P T P^{A E}(T)=I C \times \min \left\{1+\alpha\left(\frac{\sum_{t=1}^{m} S\left(T-1+\frac{t}{m}\right)}{m S(T-1)}-1\right),(1+c)\right\} .
$$

\subsubsection{PTP with High-Watermark design}

This more exotic structure records the index value at different dates during the entire term of the contract - typically at annual anniversaries -, then picks the highest value and compares it to that at the beginning of the term to calculate the growth. Here we have $R_{t}=\max _{t=1,2, \ldots, T} \frac{S(t)}{S(0)}$, and the payoff in (1.4.1) has the following expression:

$$
\operatorname{PTP}^{H W}(T)=I C \times \min \left\{1+\alpha\left(\max _{t=1,2, \ldots, T} \frac{S(t)}{S(0)}-1\right),(1+c)^{t}\right\}
$$

A Point-to-Point EIA credits the index-linked interest to the investor at the end of the term. As a result, if he wishes to surrender early or make any withdrawal before maturity, he will lose the gain from the index accordingly. One weakness of the Point-toPoint EIA with Term-End design is that it does not account for any gain in the index (if 
any) should the index value rise between times 0 and $T$ and drop dramatically right before maturity T. Similarly for the Asian-End design that doesn't account for any index-linked gain that could have happened during the first $T-1$ years of the term of the contract. However, this could be offset by the possibility of having high cap and participation rates and low spreads to allow for more "gain" to be credited. Whereas Point-to-Point EIAs with High-Watermark design are typically offered with lower cap and participation rates and higher spreads, since its clear that this design is more likely to credit more interest than the other two.

\subsubsection{Ratchet Class of EIA}

The more popular and complex class of EIAs is the Ratchet type. By definition, the word "ratchet" refers to a process that is changing steadily in an irreversible manner. A "ratchet" is also a tool that allows motion in one direction only.

A Ratchet EIA measures and earns the index return period by period over the entire term of the contract. Once credited, the interest earned is "locked-in" each period and the index value is "reset" at the end of the period, regardless of the future performance of the index. As a result and by what its name implies, a poor performance resulting from a sudden dramatic drop in the value of the index will not affect a good one preceding it that was already earned.

Two versions of the Ratchet EIA exist; the Simple Ratchet where periodic growth rates in the underlying index are added together to give the final index-linked return, and the Compound Ratchet where periodic growth rates simply compound.

In particular, consider a $T$ years Annual Ratchet EIA where index-linked gain is evaluated and credited each year. Its payoff under the Simple Ratchet case (SR) with the 
annual cap and floor rates bounding the yearly return is given by:

$$
S R=I C \times\left\{1+\sum_{t=1}^{T} \min \left(\max \left(\alpha\left(R_{t}-1\right), f\right), c\right)\right\}
$$

Similarly, the payoff under the Compound Ratchet case is:

$$
C R=I C \times\left\{\prod_{t=1}^{T} 1+\min \left(\max \left(\alpha\left(R_{t}-1\right), f\right), c\right)\right\}
$$

A GMAB option embedded in the Ratchet EIA, whether Simple or Compound, would render the payoff in this form:

$$
\begin{gathered}
\max \left(S R, I C \times \beta(1+g)^{T}\right), \\
\text { or } \max \left(C R, I C \times \beta(1+g)^{T}\right),
\end{gathered}
$$

respectively.

In both cases, the crediting method that determines the annual growth in the index $R_{t}$ differ depending on the design used.

\subsubsection{Ratchet with Term-End point design}

Under the Ratchet class of EIAs, $R_{t}$ is commonly expressed using the Term-End point design, that is by comparing the index level at the beginning and ending of each anniver-

sary year following the purchase date. Hence we have $R_{t}=\frac{S(t)}{S(t-1)}$ for $t=1,2, \ldots, T$, and the payoffs in (1.4.3) and in (1.4.4) become:

$$
S R^{T E}=I C \times\left\{1+\sum_{t=1}^{T} \min \left(\max \left(\alpha\left(\frac{S(t)}{S(t-1)}-1\right), f\right), c\right)\right\},
$$

and

$$
C R^{T E}=I C \times\left\{\prod_{t=1}^{T} 1+\min \left(\max \left(\alpha\left(\frac{S(t)}{S(t-1)}-1\right), f\right), c\right)\right\} .
$$




\subsubsection{Ratchet with High-Watermark design}

A more complex crediting method under the Ratchet class comes with the High-Watermark design applied during each year (or more generally each period). That is, the yearly credited index gain is calculated by measuring the index value $m$ times per year then picking the highest value and comparing it to that at the beginning of the year. In this case we have $R_{t}=\max _{i=1,2 \ldots, m} \frac{S\left(t-1+\frac{i}{m}\right)}{S(t-1)}$ for $t=1,2, \ldots, T$, and the payoffs in (1.4.3) and in (1.4.4) become:

$$
S R^{H W}=I C \times\left\{1+\sum_{t=1}^{T} \min \left(\max \left(\alpha\left(\max _{i=1,2 \ldots, m} \frac{S\left(t-1+\frac{i}{m}\right)}{S(t-1)}-1\right), f\right), c\right)\right\}
$$

and

$$
C R^{H W}=I C \times\left\{\prod_{t=1}^{T} 1+\min \left(\max \left(\alpha\left(\max _{i=1,2 \ldots, m} \frac{S\left(t-1+\frac{i}{m}\right)}{S(t-1)}-1\right), f\right), c\right)\right\} .
$$

\subsubsection{Index Averaging:}

Another variation for calculating $R_{t}$ is by averaging the index growth during each year, such that

$$
{ }_{a} R_{t}^{(m)}=\sum_{i=1}^{m} \frac{S\left(t-1+\frac{i}{m}\right)}{m S(t-1)}
$$

in the case of arithmetic averaging, and

$$
{ }_{g} R_{t}^{(m)}=\left[\prod_{i=1}^{m} \frac{S\left(t-1+\frac{i}{m}\right)}{S(t-1)}\right]^{\frac{1}{m}}
$$

in the case of geometric averaging.

Index averaging can also be applied to the Point-to-Point class of EIAs. It is often used to reduce the cost of the contract given that it lowers the index-linked interest earned and offers partial immunization against the index market volatility.

The advantage of a Ratchet EIA is that, due to the "lock-in" feature, it ignores any 
decline in the index levels during periods of poor index performance, and credits only positive index-linked returns. It may also credit more interest than other designs when the underlying index price makes a lot of wide fluctuations. Additionally, since interest is earned periodically, the Ratchet design is more likely than others to give investors access to their investment gains before the end of the term. However, it follows that this drives writers of such contracts to offer lower cap and participation rates and higher spreads than other types of EIAs as a way to limit their liabilities and reduce the cost of the contracts. 


\section{Chapter 2}

\section{The Black and Scholes Model}

\section{$2.1 \quad$ Introduction}

The Black-Scholes model was first introduced in 1973 and presented an analytic model that sets a fair market price for European-style options. The paper was written by Fischer Black and Myron Scholes, entitled "The Pricing of Options and Corporate Liabilities ", and was published in the Journal of Political Economy. The model was further developed by Robert Merton in "Theory of Rational Option Pricing". Much later, in 1997, Scholes and Merton were awarded the Nobel Prize in Economics for their revolutionary work in the industry of quantitative finance.

Due to its simplicity, the Black-Scholes model is still extensively used in modeling stock price fluctuations and valuing more complicated types of financial derivatives. We shall refer to the Black-Scholes model not only as a pricing formula for the standard options, but rather a financial framework that outlines the economy in general.

In this chapter we present the pricing, under the Black and Scholes (1973) model, of contingent claims who's payoffs are random but fixed at a certain point in time. Zero coupon bonds and European options are examples of such assets. 


\subsection{The Black-Scholes Economy}

A state contingent claim is a contract whose payoff is contingent - or dependent - on future states of the market. The set of all possible outcomes is defined by the sample space $\Omega$. For example, the experiment of flipping a coin one time has a total of two possible states: state one, in which the outcome is a head, and state two in which the outcome is a tail. A state-contingent claim can be a bet on heads, and would have a payoff for each possible future state, in this case, a payoff of $\$ 1$ if state one occurs (the outcome of the coin flip is a head), and a payoff of $\$-1$ if state two occurs (the outcome is a tail).

Note that in a complete market, it is always possible to replicate using a position that would result in the same payoff regardless of the future state. The assumption of complete markets implies that all claims are attainable. In other words, one could always perfectly replicate the payoff, at any time $t$, of every state-contingent claim, hedging by a self-financing trading strategy which satisfies an admissibility condition imposed in order to rule out the possibility of arbitrage.

A self-financing trading strategy is defined as follows: Consider an investor with an initial wealth of $\$ X$ constructs a portfolio of derivative securities, that could be a combination of a number of shares bought and a number of options sold for example. The value of the portfolio changes in time as a result of changes in the price and/or portion of the risky and non-risky assets. In this case, the investor's trading strategy is said to be self financing if changes in the portion of these assets is done such that no funds are added (or withdrawn) from the initial investment. That is, the cost of buying more units of a security is fully financed by selling some units of another security from the same portfolio.

The mere existence of this strategy implies that the initial amount of money needed to construct will equal the current price of the contingent claim (or derivative). Naturally, one would ask himself the question: could a claim be attainable through a certain number of different strategies, and thus leading to different prices? Or is there a unique hedging 
strategy generating each contingent claim, thus leading to a unique price? The answer to that question will be found at the end of this section.

\subsubsection{Continuous Stochastic Processes}

A stochastic process is a collection of random variables indexed by time. In financial models, trading strategies, securities prices, exchange rates, etc... are usually modeled as stochastic processes.

A definition of a continuous stochastic process could be found in Nielsen (1999), and is presented as follows:

Definition 2.1. Given the probability space $(\Omega, \mathcal{F}, P)$, where $\mathcal{F}$ is a $\sigma$-algebra on $\Omega$, then $X=\{X(t), t \geq 0\}$ is a continuous-time stochastic process, such that for each fixed $t \in[0: \infty)$, the mapping $X(t): \omega \mapsto X(\omega, t): \Omega \rightarrow \mathbb{R}$ is measurable, and that for each fixed $\omega \in \Omega$, the function $t \longmapsto X(\omega, t):[0, T] \rightarrow \mathbb{R}$ is called a sample path of the process.

Example: let $V(t)$ be a random variable representing the value at time $t$ of a trading strategy or portfolio, whose payoff is $V(T)$ at maturity time $T$. Then the process $V=\{V(t), 0 \leq t \leq T\}$ is a continuous-time stochastic process.

A very famous example of a continuous-time stochastic process is Brownian motion, which plays a fundamental role in stochastic calculus and financial mathematics, and will be introduced later.

\subsubsection{Martingales}

Martingale processes are known to model the total wealth of a player in a fair game. The game is fair in the sense that the player's wealth tend to remain constant over time. That is, the expected value of the total wealth at any future time $t+s$, given the history of the wealth process up to and including its current value at time $t$, will be equal to its current wealth value at time $t$. 
Let $\mathcal{F}_{t}$ be the filtration which represents a collection of information that increases in time, such that $\mathcal{F}_{s} \subset \mathcal{F}_{t}$ for all $s<t$. This increasing sequence means that there is no loss of information.

Consider any continuous-time stochastic process $X$. Then $X$ is adapted to the filtration $\mathcal{F}_{t}$, if, for every $t$, each random variable $X(t)$ is $\mathcal{F}_{t}$-measurable. That is the value $X(t)$ can be determined by - and depends only on - the filtration $\mathcal{F}_{t}$ that represents all the information given up to time $t$. In other words, an adapted stochastic process cannot "see into the future".

Then we can say the following.

Definition 2.2. The adapted process $X$ is a martingale if:

$\mathcal{F}_{t} \quad$ is an increasing family of sigma-algebras.

$\mathbb{E}\left[X(t) \mid \mathcal{F}_{s}\right]=X(s)$ for all $0 \leq s<t$.

Each $X(t)$ is $\quad \mathcal{F}_{t}$ measurable and $\mathbb{E}[|X(t)|]<\infty$ for all $t \in[0, \infty]$.

Or equivalently, $\mathbb{E}\left[X(t)-X(s) \mid \mathcal{F}_{s}\right]=0$. Also, the process $X$ is said to be a submartingale (respectively super-martingale) if the equality is replaced by $\geq$ (respectively $\leq)$. The condition of the finite mean is important to guarantee the existence of the conditional expectations.

Note that a process that is both a sub-martingale and super-martingale is a martingale.

Remark: It is important to note that, by definition, a martingale process has a constant expectation over time. For instance, let $s=0$ in (2.2.1), then

$$
\mathbb{E}\left[X(t) \mid \mathcal{F}_{0}\right]=X(0) \quad \text { for all } \quad 0 \leq t \leq T
$$

where $X(0)$ is the value of the process at time 0 . 


\subsubsection{The Martingale Valuation Principle}

First of all, when dealing with expectation, it is crucial to determine the measure under which the expectation is taken. Denote by $\mathbb{P}$ the physical measure which gives the actual probability of occurrence of various states of the world. Denote by $\mathbb{Q}$ the riskneutral measure, also known as the equivalent martingale measure. $\mathbb{Q}$ is a probability measure such that future outcome probabilities are adjusted to incorporate all investors' risk premiums, and where the expected rate of return of all derivative securities is the same, and is equal to the risk free rate, therefore they do not incorporate any risk premium. Thus an investor is indifferent towards the choice of any risky asset.

The risk-neutral probability measure $\mathbb{Q}$ is said to be equivalent to the probability measure $\mathbb{P}$, if both measures are defined on the same measurable space $(\Omega, \mathcal{F})$, and

$$
\mathbb{P}(\omega)>0 \Leftrightarrow \mathbb{Q}(\omega)>0 \text { for all } \omega \in \Omega \text {. }
$$

In other words, although the probability values of individual events $\omega$ may not be same within both measures, $(\mathbb{P}(\omega) \neq \mathbb{Q}(\omega)$ for all $\omega \in \Omega)$, but equivalent measures must always agree on which events are possible (assigned strictly positive probabilities), and which events are impossible (assigned zero probabilities), as stated in Kwok (2008).

According to the Fundamental Theorem of Asset Pricing, the condition of no-arbitrage is equivalent to the existence of the risk-neutral probability measure $\mathbb{Q}$.

An arbitrage opportunity relies in a self-financing trading strategy that requires an initial investment $V(0)=\$ 0$, has a zero probability of losing money, and some positive probability of making money. Thus having $V(t) \geq 0$ and $\mathbb{E}[V(t)]>0$ for all $t$. The strictly positive expectation implies that there exists at least one positive payoff.

Theorem 2.1. In complete markets, arbitrage opportunities do not exist if and only if there is a probability measure $\mathbb{Q}$, equivalent to the real measure $\mathbb{P}$, such that under $\mathbb{Q}$, the 
discounted price process of risky assets is a martingale.

Proof. See Harrison and Pliska (1981)

Based on this important theorem, we can write,

$$
\mathbb{E}^{\mathbb{Q}}\left[V(t) v(0, t) \mid \mathcal{F}_{s}\right]=V(s) v(0, s) \quad \text { for all } \quad 0 \leq s<t \leq T
$$

where $v(0, t)$ is a discounting factor from time $t$ to 0 , and $V(t) v(0, t)$ is the discounted price process. Thus, the unique price $V(0)$ of any derivative security is calculated by discounting the expected value of its future payoff $V(T)$ under the unique risk-neutral probability measure $\mathbb{Q}$.

Let $s=0, t=T$, and take the expectation of the discounted price process $V$ under $\mathbb{Q}$ in $(2.2 .2)$, you get

$$
\mathbb{E}_{\mathbb{Q}}\left[V(N) v(0, N) \mid \mathcal{F}_{0}\right]=V(0)
$$

where $V(0)$ is the value at time 0 of the trading strategy having payoff $V(N)$ at expiration $N$.

Since the discounted price process is a martingale, it has a constant expectation equal to its price. In addition, since in complete markets all claims can be replicated by a selffinancing trading strategy with an initial investment equal to the price of the claim, then it is impossible to start with an initial wealth of $\$ 0$ and end up having a strictly positive payoff with positive probability, unless there is also a positive probability of having a strictly negative payoff, in order to keep the expectation 0 at all times, and thus rules out the possibility of arbitrage opportunities.

In fact, in arbitrage-free markets, the Law of One Price applies for all derivatives securities - that is, securities with the same payoff have the same price, and the same security has the same price in all markets -, or else, an investor would benefit from the difference in price of the same derivative security in different markets, $\mathrm{A}$ and $\mathrm{B}$ for example, to make immediate risk-free profit by buying low from one market, say A, and selling high 
in another market, B. Thus, this profit from price discrepancy is considered as doing an arbitrage.

Finally, the unique price $V(0)$ implies the uniqueness of the self-financing trading strategy of every contingent claim, under the unique risk-neutral measure $\mathbb{Q}$.

\subsection{The Model's Assumptions}

While valuing derivative securities whose payoff is dependent on the stock price, Black and Scholes (1973) impose some ideal conditions on both the financial market and the stock.

(a) Dividends are not paid. The first assumption states that stocks pay no dividends or any other distributions during the life of the contract, or in other words, stock's dividends are not distributed. Since most companies pay dividends to their shareholders, this assumption could be easily relaxed by subtracting the discounted value of future dividends from the stock price.

(b) All fractions of the price of any security can be borrowed, bought or held at the short term interest rate $r$.

(c) Short term interest rates are known and remain constant through time. Let $r$ be the continuously compounded yearly interest rate, then the discount factor $v(0, t)$ can be written this way:

$$
\begin{aligned}
v(0, t) & =e^{-\int_{0}^{t} r(t) d t} \\
& =e^{-t r}
\end{aligned}
$$

Note that this same interest rate $r$ is used for both lending and borrowing.

In contrast to the deterministic interest rate $r$, and for more details on stochastic interest rate models and bond pricing, refer to Brigo and Mercurio (2007). 
(d) There are no restrictions or penalties on short selling. The seller, not owning the security, settles with the buyer by agreeing on paying him an amount equal to the price of the security, set by the buyer, at some future time.

(e) The market is efficient. The efficient market hypothesis is a strong assumption that leads to other subsequent ones. Fama (1969) states the following three sufficient, but not necessary, conditions for capital market efficiency:

(a) The market is frictionless; that is, the bid and ask spread of trading securities is zero, i.e. transactions do not incur any fees or costs.

(b) All investors have free access to all available information in the market, making it a fair game for everyone.

(c) All investors agree on the implication of current information for the current price and distributions of each security's future prices.

Efficient financial markets are frictionless markets in which investors have access to all available information which is reflected through the price of assets and securities. In fact, the main importance of the assumption of efficient markets is that they rule out the possibility of arbitrage. The fast spread of new information makes the prices change quickly to settle around the equilibrium price, which fully incorporates the launching of new information, making it a fair and unique market price. This implies that the study of past stock prices will not be relevant in the prediction of future movements of stock prices. More generally, technical stock analysis, as well as fundamental analysis, which is the measurement of a security's intrinsic value, fail to provide investors or analysts with (1) an effective pattern that indicates future stock performance, nor (2) useful insights on the status of the current stock price (that is whether its undervalued or overvalued), as explained by Malkiel (2003). As a result, successive price changes, or more generally, successive periodic returns are independent. 
This assumption is mathematically equivalent to the Markovian property. Let $R=$ $\{R(t), t \geq 0\}$ be the return on the stock price process for $t$ in $[0, \infty)$. Then $R$ is a Markov process such that

$$
\mathbb{P}\{R(t)=x \mid R(u), 0 \leq u \leq s\}=\mathbb{P}\{R(t)=x \mid R(s)\}
$$

for any $0 \leq s<t$.

Additionally, it is assumed that these consecutive price changes, or returns, are also identically distributed, hence the last assumption:

(f) The stock price process $S$ follows a Geometric Brownian Motion with drift (Random Walk in continuous time).

\subsection{The Stock Price Process}

\subsubsection{Standard Brownian Motion Process}

Consider a continuous-time stochastic process $B=\{B(t), t>0\}$ with the following properties:

- $B(0)=0$ with probability one.

- For $0 \leq t_{0}<t_{1}<\ldots<t_{n-1}<t_{n}<\infty$, the random variables $B\left(t_{1}\right)-B\left(t_{0}\right), \ldots, B\left(t_{n}\right)-$ $B\left(t_{n-1}\right)$ are independent, and we say that this process has independent increments.

- For $0 \leq s \leq t<\infty$, the increment $B(t)-B(s)$ is normally distributed with mean 0 and variance $t-s . B(t)-B(s) \sim N(0, t-s)$.

Then this particular process is called a one-dimensional standard Brownian motion process, also known as a Wiener process. 
According to Nielsen (1999), this process represents the basic building block of $98 \%$ of all finance theory in continuous times. It is also of fundamental importance in both the theory and applications of probability. The mathematical existence of such a process is verified by the mathematician Norbert Wiener in 1923, see Wiener (1923).

One can deduce the following characteristics of this process from its previously mentioned properties:

- For each $t \in[0, \infty)$, the distribution of the random variable $B(t)$ is normal with mean 0 and variance $t$. $B(t) \sim N(0, t)$. It follows that the unconditional mean of the value of the process at each point in time $t$, given the filtration $\mathcal{F}_{0}$, is zero.

- For $0 \leq s<t<\infty$, the distribution of the random variable $B(t)-B(s)$ is the same as the distribution of the random variable $B(t-s)$. Thus, the distribution of the increments $B(t)-B(s)$, for all $0 \leq s<t<\infty$, depends only on the time difference $t-s$ between $s$ and $t$, and not on the actual times $(s, t)$. We say that this process is time-homogeneous, or has stationary increments.

- Any two increments over non-overlapping time intervals are independent.

- $\operatorname{cov}(B(s), B(t))=\min (s, t)$.

Figure 2.1 represents an approximation of three sample paths of one-dimensional standard Brownian motion process, along with the standard deviation curves that delineate a range of plus or minus one standard deviation around the mean, $0 \pm \sqrt{t}$. Values on the horizontal axis represent the time $t$, and the vertical axis measures the values of the Brownian motion.

These sample paths are generated as follows: first, 180 independent standard normal random variables are randomly generated and multiplied by $\sqrt{\frac{1}{30}}$ in order to change their variance from 1 to $\frac{1}{30}$. Each number represents the increment of the Brownian motion over the time interval $\left[t, t+\frac{1}{30}\right]$. Therefore, the cumulative sum of these random variables 


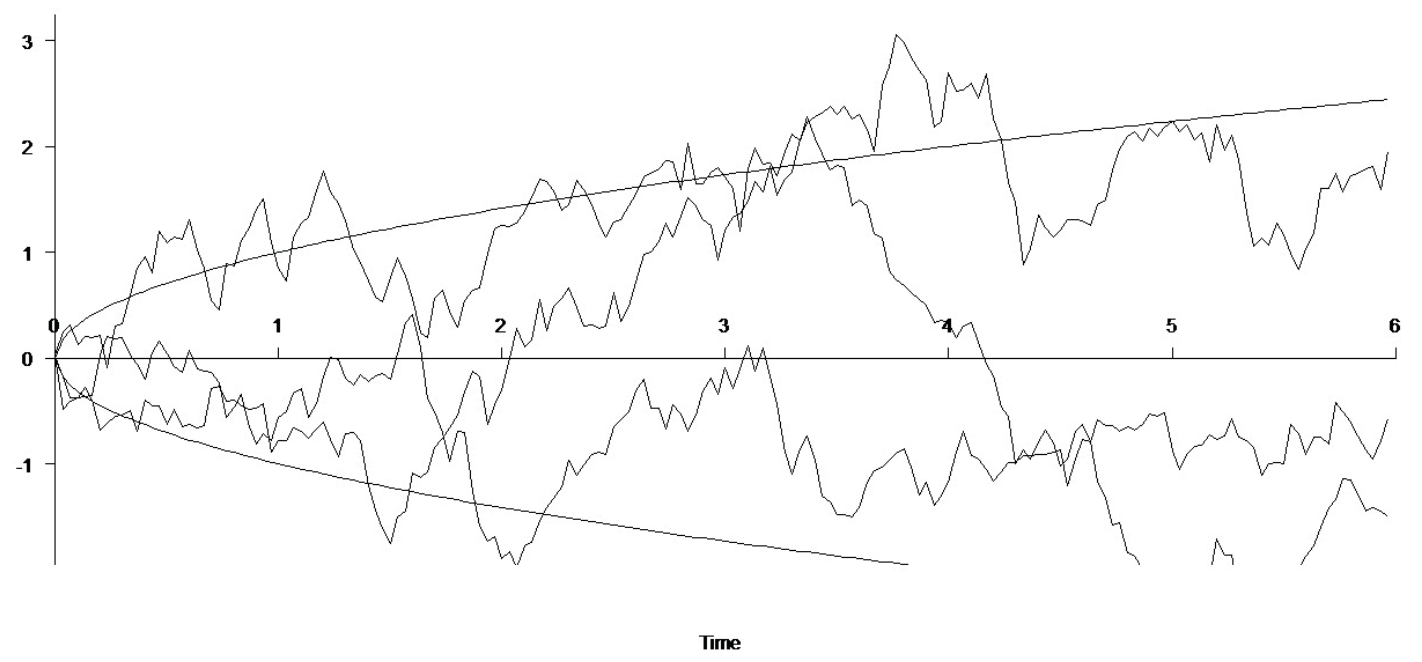

Figure 2.1: Three samples paths of standard Brownian motion processes.

gives the values of the standard Brownian motion process, sampled at discrete points in time that are $\frac{1}{30}$ units apart. Finally, these values are then joined by a straight line to give an approximation of one sample path.

To wrap up, the standard Brownian motion is a continuous-time stochastic process that starts at 0 , its increments are independent and not correlated with each other and have means of zero and variances of 1 per unit of time. Intuitively, stock prices cannot be modeled by this process alone since they do not start from a null value, and normally their increments have positive means and will probably be correlated.

The standard Brownian motion process is a special case of a more general and flexible continuous stochastic process: the Generalized Brownian Motion process, where the process may start with a value different than zero, and where its increments have means, variances and covariances that are still constants, but not necessarily zero, one and zero, respectively. 


\subsubsection{Generalized Brownian Motion Process}

The mathematical theory of Brownian motion was first introduced by the French mathematician Bachelier (1900) in a thesis about option pricing submitted to the Academy of Paris, five years before Einstein's classic 1905 paper.

Let $G=\{G(t), t>0\}$ be a Generalized Brownian Motion. Then $G$ is a continuoustime stochastic process having the following properties:

- $G(0)$ is deterministic.

- For $0 \leq t_{0}<t_{1}<\ldots<t_{n-1}<t_{n}<\infty$, all increments $G\left(t_{1}\right)-G\left(t_{0}\right), \ldots, G\left(t_{n}\right)-$ $G\left(t_{n-1}\right)$ are independent.

- For $0 \leq s \leq t<\infty$, the increment $G(t)-G(s)$ is normally distributed with mean $(t-s) \mu$ and variance $(t-s) \sigma^{2}$.

Then $G(t)-G(s) \sim N\left((t-s) \mu,(t-s) \sigma^{2}\right)$.

It follows that a generalized Brownian motion process can be constructed by rescaling a standard Brownian motion by a constant $\sigma$ and adding an initial value $G(0)$ and a linear increment $\mu$. This relationship is summarized by the following equation:

$$
G(t)=G(0)+\mu t+\sigma B(t)
$$

where $\quad \mathbb{E}[G(t)]=G(0)+\mu t, \quad \mathbb{V}[G(t)]=\sigma^{2} t \quad$ and $\quad G(t) \sim N\left(G(0)+\mu t, \sigma^{2} t\right)$.

Now since $G(t)$ is normal random variable, it can take negative values with positive probability whereas stock prices can never be negative due to their limited liability, they are not well described either by a generalized Brownian motion process. In addition, this process has additive increments - hence the term arithmetic -, in contrast to the stock prices' increments that ought to be multiplicative. In that same sense, Samuelson (1965) explains in his paper how Bachelier's arithmetic Brownian motion is not suitable to price 
derivative securities, and introduces a better hypothesis for an economic model, which is the Geometric or Relative Economic Brownian motion.

\subsubsection{The Stock Price as a Geometric Brownian Motion Process}

Let $S(t)$ represent the stock price at time $t$, and $S=\{S(t)>0,0 \leq t \leq T\}$ be the stock price process between times 0 and $T$. Then the appropriate model that should be used to describe the random evolution of the stock price process $S$ is the Geometric Brownian motion of the form $S(t)=S(0) e^{G(t)}$, where $S(t)$ satisfies the following stochastic differential equation:

$$
d S(t)=\mu S(t) d t+\sigma S(t) d B(t)
$$

where $\mu$ and $\sigma$ are both constants representing the mean rate of return (or drift) and volatility of the stock prices, respectively.

The solution to this equation can be found using Itô calculus, and is given by

$$
S(t)=S(0) \exp \left[\left(\mu-\sigma^{2} / 2\right) t+\sigma B(t)\right] .
$$

Then to get the distribution of $S(t)$ we can write: $\ln S(t)=\ln S(0)+\left(\mu-\sigma^{2} / 2\right) t+$ $\sigma B(t)$, which is a generalized Brownian motion process with initial value $\ln S(0)>0$, and the conditional distribution of $\ln S(T)$ given $S(t)$ is normal with mean $\ln S(t)+(\mu-$ $\left.\sigma^{2} / 2\right)(T-t)$ and variance $\sigma^{2}(T-t)$. That is,

$$
\ln S(T) \mid S(t) \sim N\left(\ln S(t)+\left(\mu-\sigma^{2} / 2\right)(T-t), \quad \sigma^{2}(T-t)\right) .
$$

It follows that $S(T) \mid S(t)$ is a log-normal random variable with the same parameters:

$$
S(T) \mid S(t) \sim L N\left(\ln S(t)+\left(\mu-\sigma^{2} / 2\right)(T-t), \quad \sigma^{2}(T-t)\right),
$$


and

$$
\begin{aligned}
\mathbb{E}\left[S(T) \mid \mathcal{F}_{0}\right] & =\exp \left(\ln S(0)+\left(\mu-\sigma^{2} / 2\right) T+\sigma^{2} T / 2\right) \\
& =S(0) e^{\mu T}
\end{aligned}
$$

Recall that, according to the Fundamental Theorem of Asset Pricing, the discounted price process of a stock is a martingale under the risk-neutral measure $\mathbb{Q}$, and a martingale process has a constant expectation over time equal to its value at time zero. Under $\mathbb{P}$ we have $\mathbb{E}^{\mathbb{P}}\left[e^{-r T} S(T) \mid \mathcal{F}_{0}\right]=S(0) e^{(\mu-r) T}$. Therefore, under the $\mathbb{Q}$ measure, the discounted stock price process $e^{-r T} S_{T}$ is a martingale relative to $\mathcal{F}$ such that $\mathbb{E}^{\mathbb{Q}}\left[e^{-r T} S(T) \mid \mathcal{F}_{0}\right]=$ $S(0)$ if and only if $\mu=r$, that is, the drift $\mu$ of the stock price process $S$ is the risk-free interest $r$, and $S(t)$ is the solution to the stochastic differential equation

$$
\begin{aligned}
d S(t) & =r S(t) d t+\sigma S(t) d B(t), \quad \text { whose solution is } \\
S(t) & =S(0) \exp \left[\left(r-\sigma^{2} / 2\right) t+\sigma B(t)\right], \quad \text { and } \\
S(T) \mid S(t) & \sim L N\left(\ln S(t)+\left(r-\sigma^{2} / 2\right)(T-t), \quad \sigma^{2}(T-t)\right) .
\end{aligned}
$$

\subsubsection{The Money Market Account}

Under the Black-Scholes option pricing model, there are two main assets: the risky one which is the stock whose dynamics are presented in (2.4.2), and a risk-less asset which is the money market account.

The money market account is comprised of short term risk-less securities, earning the continuously compounded risk-free interest rate $r$. Examples of such very liquid instruments are T-Bills and short-term Bonds. 
Let $M=\{M(t), 0 \leq t<\infty\}$ be the continuous price process of a unit of the money market account. Then

$$
M(t)=M(0) e^{r t}
$$

with a constant initial value $M(0)>0$. Observe that $M(t)$ satisfies $d M(t)=r M(t) d t$, and the process $M$ is a deterministic, degenerate, generalized Brownian motion process.

\subsection{Pricing European Call Options in BS}

A Call (/ Put) option with $T$ years until maturity is a financial derivative security, or simply a contract, that gives its buyer the right but not the obligation to buy (/ sell) an asset for a predetermined strike price $K$ within the period of $T$ years. Should the buyer decide to exercise his right, the seller of the Call would be obliged to sell (/ buy) the underlying asset for $\$ K$, the amount agreed upon at the inception of the contract, regardless of the underlying's current price. The period of time during which the buyer is allowed to exercise the option depends on the style of that option. European-style options gives the buyer the right to exercise only at maturity of the contract $T$. However, American-style options may be exercised at any time before expiration of the contract.

Consider a rational investor who buys one unit of a Call option on one share of the stock $S$. Naturally, the investor will exercise the option at maturity $T$ by buying the stock for $\$ K$ if and only if the underlying price at maturity $S(T)$ is higher than or equal to $K$, $S(T) \geq K$. Having done that, the investor would go and sell that same share of stock at the market price $S(T)$, thus making a profit of $S(T)-K(>0)$. Or else, should $S(T)$ drop below $\$ K$, the option will expire worthless. 
Let $\Lambda^{C}$ be the payoff of a Call option, then

$$
\begin{aligned}
\Lambda^{C} & =\max \{S(T)-K, 0\} \\
& = \begin{cases}S(T)-K & \text { if } S(T) \geq K \\
0 & \text { otherwise. }\end{cases}
\end{aligned}
$$

To price the Call option is equivalent to ask how much the investor is willing to pay at time 0 to acquire the contract. Given that the option's payoff depends on the price $S(T)$ which is a random quantity at time 0 , then the investor purchasing the option should bear the risk associated with the stock. Therefore, his valuation of the option clearly depends on his attitude towards risk-bearing, and different investors with different risk appetite would be willing to pay different amounts to acquire such a contract. However, recall that, operating under the Black-Scholes framework, there exists a unique rational price for the Call option, regardless of one's risk attitude.

\subsubsection{Pricing Under the Martingale Valuation Principal}

One way to obtain this price is through taking the expected value, under the riskneutral measure $\mathbb{Q}$, of the payoff discounted back to time zero by the risk-free interest rate $r$, as presented by Harrison and Pliska (1981).

Let $V_{t}^{C}$ be the value of a Call option at time $t$ whose payoff is $\Lambda^{C}$ on the underlying $S(t)$ modeled as in (2.4.3). Then

$$
V_{t}^{C}=\mathbb{E}^{\mathbb{Q}}\left[e^{-r(T-t)} \Lambda^{C} \mid \mathcal{F}_{t}\right]
$$

Let $f_{S(T)}$ be the density function of the log-normal random variable $S(T)$, then we have: 


$$
\begin{aligned}
\mathbb{E}^{\mathbb{Q}}\left[e^{-r(T-t)} \Lambda^{C} \mid \mathcal{F}_{t}\right]= & e^{-r(T-t)} \int_{K}^{\infty}(x-K) f_{S(T)}(x) d x \\
= & e^{-r(T-t)} \int_{K}^{\infty} x f_{S(T)}(x) d x-K e^{-r(T-t)} \int_{K}^{\infty} f_{S(T)}(x) d x \\
= & e^{-r(T-t)} \int_{K}^{\infty} \frac{1}{\sqrt{2 \pi} \sigma \sqrt{T-t}} \exp \left\{\frac{-\left(\ln \frac{x}{S(t)}-\left(r-\sigma^{2} / 2\right)(T-t)\right)^{2}}{2 \sigma^{2}(T-t)}\right\} d x \\
& -K e^{-r(T-t)} \mathrm{P}_{L N}(S(T)>K) .
\end{aligned}
$$

Applying the change of variable $y=\ln \frac{x}{S(t)}$ in the first term, we get:

$$
\begin{aligned}
& V_{t}^{C}= S(t) \Phi\left(\frac{\ln \frac{S(t)}{K}+\left(r+\sigma^{2} / 2\right)(T-t)}{\sigma \sqrt{T-t}}\right) \\
&-K e^{-r(T-t)} \Phi\left(\frac{\ln \frac{S(t)}{K}+\left(r-\sigma^{2} / 2\right)(T-t)}{\sigma \sqrt{T-t}}\right) \\
& V_{t}^{C}=S(t) \Phi\left(d_{1, t}\right)-K e^{-r(T-t)} \Phi\left(d_{2, t}\right)
\end{aligned}
$$

where $\Phi$ is the cumulative distribution function of a standard normal random variable, and $d_{1, t}$ and $d_{2, t}$ are given as follows:

$$
d_{1, t}=\frac{\ln \frac{S(t)}{K}+\left(r+\sigma^{2} / 2\right)(T-t)}{\sigma \sqrt{T-t}}
$$

and

$$
\begin{aligned}
d_{2, t} & =\frac{\ln \frac{S(t)}{K}+\left(r-\sigma^{2} / 2\right)(T-t)}{\sigma \sqrt{T-t}} \\
& =d_{1, t}-\sigma \sqrt{T-t}
\end{aligned}
$$

Then the unique rational price at time 0 of the Call option can be found by setting $t=0$ in the equations of $V_{t}^{C}, d_{1, t}$ and $d_{2, t}$ to get the famous Black-Scholes pricing formula:

$$
V_{0}^{C}=S(0) \Phi\left(d_{1,0}\right)-K e^{-r T} \Phi\left(d_{2,0}\right)
$$




\subsubsection{Pricing Under the Replicating Portfolio Method}

A similar approach to price the Call option is through finding the initial value required to construct a trading strategy $\psi$ that yields the same payoff and has the same cash flow as that of the Call option at any time $t$ between 0 and $T$. Thus the portfolio consisting of such a duplicating strategy is known as the replicating portfolio.

Assuming the Black-Scholes framework, this replicating portfolio consists only of stocks and money market accounts. Then we shall refer to this strategy as $\psi=\left\{\psi_{S}, \psi_{M}\right\}$, where $\psi_{S}$ is the total number of shares of stock held in the portfolio, and $\psi_{M}$ is the total number of risk-less securities held in the money market account.

Let $V_{t}^{\psi}$ be the market value of the replicating portfolio at time $t$, then

$$
V_{t}^{\psi}=\psi_{S}(t) S(t)+\psi_{M}(t) M(t)
$$

Observe that to always have $V_{t}^{\psi}=V_{t}^{C}$ from (2.5.2), then the following must hold for all $0 \leq t \leq T$

$$
\begin{aligned}
\psi_{S}(t) & =\Phi\left(d_{1, t}\right), \quad \text { and } \\
\psi_{M}(t) M(t) & =-K e^{-r(T-t)} \Phi\left(d_{2, t}\right) \\
& =V_{t}^{C}-\psi_{S}(t) S(t),
\end{aligned}
$$

where the last equation defining the amount invested in the risk-free asset implies that the condition of the self-financing trading strategy is satisfied by $\psi$.

Remark: Notice that the portion of money invested in the risky asset, $\psi_{S}(t)$, at any time $t$, is nothing but the first derivative of the Call option price with respect to the current price of the underlying stock $S(t)$. The idea behind this relationship will be presented in the following chapter. 


\section{Chapter 3}

\section{The Hedging Strategy}

\subsection{Introduction}

Hedging in finance is the act of strategically trading financial instruments with the objective of offsetting, or eliminating as much as possible, the exposure to risks associated with an investor's initial position in assets or financial instruments already owned. Consider for example an investor having a long position in an option on the S\&P 500 index, and at the same time a short position in some shares of the S\&P 500 stocks. The possibility that the S\&P 500 stock price decreases, leading to a decrease in the value of the option, will result in a loss from the investor's long position in that option. However, this potential loss will be offset by a gain resulting from the investor's short position in the value of the S\&P 500 index shares. In this way, we can say that the investor has hedged his long position in the option by an opposite - short - position in the underlying asset shares.

Why to hedge? In this example, the option's value at any time $t$ from inception of the contract until maturity depended on the price of its underlying asset at maturity. In general, the price of derivative securities can depend on a certain number of varying parameters that fluctuates randomly through time, then the derivative's value and its payoff will also be random and change accordingly. As a result, this randomness represent a risk for the issuers, therefore they usually take action by hedging. 
A hedging strategy that totally eliminates the risk in a position is said to be perfect. Otherwise, in the case when there is still a portion of the risk borne by the issuer, then it is said to be partial. Different hedging methods exist and they depend on the status of the market, whether its complete or not, as well as on whether our model is a continuous-time or a discrete-time model. In complete market conditions, one can achieve a perfect hedge by perfectly replicating the initial position. Whereas in incomplete markets, the hedge can only be partial and thus aims at only minimizing the exposure to risk rather than completely removing it.

Broadly speaking, a hedge can be either static or dynamic. A static hedge is when the hedging portfolio is constructed at time zero such that the number of securities in it remain fixed. Whereas a dynamic hedge is when the hedging portfolio is rebalanced through time. In particular, dynamic hedging can be split into two main categories; local and global hedging. Dynamic local hedging techniques such as the moving-based local hedging (in particular the Delta-hedging method), the time-based local hedging (used in Ederington (1979)), and the local risk minimization (developed by Follmer and Schweizer (1988)), target the risk in each small time period independently. In contrast, dynamic global hedging techniques such as global quadratic hedging (developed by Schweizer (1995)), global risk minimization (see Xu (2006) and Godin et al. (2014)), and super-replication (see Karoui and Quenez (1995)), consider the aggregate risk jointly from one period to another.

So far in this thesis, we have only described the conditions of complete markets in which we can perfectly hedge our initial position by replicating the derivative, hence the importance of the arbitrage-free self-financing replicating strategy $\psi$. Thus this replicating strategy does not only allow us to price, but also to hedge any financial security whose payoff is dependent on certain parameters (or assets), in particular the stock price $S_{t}$ by using the Delta-hedging method. However, in Section 3.2.2, we will show an improvement (by incorporating the Gamma-Hedging) that accounts for the incompleteness of our market resulting from the application of a continuous time model to discrete time. 
How to hedge? Recall that by definition, the value $V_{t}^{\psi}$ of the portfolio replicating any particular derivative always equals the value $V_{t}$ of that derivative. Hence, to hedge your position in any derivative, you must simultaneously take an "opposite position" in the corresponding replicating portfolio. Consequently, should you incur any loss resulting from a movement in the derivative's price by holding the initial position, this loss will be simultaneously offset by a gain of the same amount from holding the opposite position in the replicating portfolio at any time $t$ from inception till maturity of the contract. More specifically, our arbitrage-free model implies that the derivative's price at time zero is equal to the cost of setting up the replicating portfolio. Hence after defining our pricing framework, we are able to set the initial capital available for constructing the hedging portfolio equal the price of the derivative. Thus the issuer of the derivative's contract will use the proceedings to set up his hedging portfolio, and no injection of additional capital would be required.

For the purpose of our thesis, we resort to a moving base dynamic local hedging technique, where the replicating strategy $\psi$ of the hedging portfolio will be constructed by using the Greeks. The idea behind this is the following: our goal is to find a way for constructing a replicating portfolio whose value $V_{t}^{\psi}$ moves in the same direction and by the same proportion as that of $V_{t}$, at any time $t$ between 0 and $T$, as the time-varying parameters change. This could be done by continuously re-balancing the portfolio in order to match the sensitivities of $V_{t}$ and $V_{t}^{\psi}$ with respect to these parameters. Doing this helps determine the appropriate positions in each risky asset of the portfolio (stocks or options or combinations of both). Then with these positions being fixed, their holdings are financed through lending or borrowing, as necessary; The difference between the value of the short derivative and the risky part of the hedge is the amount to be invested in the money market account. When negative, it represents a loan taken by investors, and when positive, it represents a surplus re-invested in the money market account at the continuously compounded risk-free rate of return $r$. Additionally, the initial holdings of 
the money market account are adjusted such that the trading strategy has the desired initial amount, equal to the price of the derivative to be hedged. Hence, this ensures that $\psi$ remains arbitrage-free and self-financing.

\subsection{The Greeks}

The Greeks are mathematical quantities used to measure the sensitivities of the price of derivatives to changes in the underlying parameters on which the value of the derivative security is dependent. The word "Greeks" stems from the fact that most of these measures are denoted by Greek letters. Being vital tools in risk management, the Greeks are also known as hedge parameters, or risk sensitivities. Depending on the hedge parameter used, each component of risk can be treated independently, making it easy for the investor to manipulate and achieve a desired risk exposure by re-balancing his portfolio accordingly. The Greek measures are split into two main classes: First order Greeks, such as Delta, and second order Greeks, such as Gamma. For more details on the rest and how they can be used to hedge, see Wilmott (2007).

\subsubsection{Delta-Hedging}

The Delta-hedging strategy was first described by Thorp and Kassouf (1967). The Greek letter $\Delta$ represents a first order risk measure that indicates how exposed, or how sensitive, a derivative is to changes in the underlying stock price, while all other variables remain unchanged.

Definition 3.1. The Delta $\Delta$ is computed as the first derivative of the instrument's value, $V$, with respect to the price of its underlying asset $S$,

$$
\Delta_{t}=\frac{\partial V_{t}}{\partial S_{t}} .
$$

With this in mind, we define the Delta-hedging strategy as a way to eliminate the risk 
by exploiting the correlation between the derivative security and its underlying.

Let $\psi^{\Delta}=\left\{\psi_{S}^{\Delta}, \psi_{M}^{\Delta}\right\}$ be the Delta-hedging strategy for the derivative security in question, and denote by $V_{t}^{\Delta}$ the value of the Delta-hedging replicating portfolio consisting of the strategy $\psi^{\Delta}$. Then this portfolio is composed of $\psi_{S, t}^{\Delta}$ shares of the underlying $S$, and $\psi_{M, t}^{\Delta}$ units in the money market account $M$ at any time $t$, and we can write:

$$
V_{t}^{\Delta}=\psi_{S, t}^{\Delta} S_{t}+\psi_{M, t}^{\Delta} M_{t}
$$

Then for $V_{t}^{\Delta}$ to have the same sensitivity to changes in the stock price as that of $V_{t}$, we need to match their Deltas, such that

$$
\Delta_{t}^{\Delta}=\Delta_{t}
$$

where $\Delta_{t}^{\Delta}$ is the Delta of the Delta-hedging portfolio, and can be given by Definition 3.1 as

$$
\begin{aligned}
\Delta_{t}^{\Delta} & =\frac{\partial V_{t}^{\Delta}}{\partial S_{t}} \\
& =\frac{\partial}{\partial S_{t}}\left(\psi_{S, t}^{\Delta} S_{t}+\psi_{M, t}^{\Delta} M_{t}\right) \\
& =\psi_{S, t}^{\Delta} .
\end{aligned}
$$

The second term goes to zero since it represents the amount invested in the money market account which is insensitive to changes in the stock price.

By combining (3.2.2) and (3.2.3), we obtain the Delta-hedging strategy by fixing the number of shares bought to the Delta of the derivative to be hedged: $\psi_{S, t}^{\Delta}=\Delta_{t}$, and fixing the amount to be invested in the money market account as the difference between $V_{t}$ and the amounts invested in the risky assets, $\psi_{M, t}^{\Delta} M_{t}=V_{t}-\Delta_{t} S_{t}$. 
Finally, by replacing these values in (3.2.1) we get:

$$
\begin{aligned}
V_{t}^{\Delta} & =\Delta_{t} S_{t}+\left(V_{t}-\Delta_{t} S_{t}\right) \\
& =V_{t}
\end{aligned}
$$

which proves that the values of both position are always the same for all $0 \leq t \leq T$, and thus taking a long position for example in the hedging portfolio will always offset the risks of the short position in the derivative security.

\subsubsection{Example 1. Delta of a European Call option:}

Under the Black-Scholes model, the Delta of the European Call option at $0 \leq t \leq T$ with payoff $\Lambda^{C}$ as in (2.5.1) is the rate of change of its value $V_{t}^{C}$ in (2.5.2) with respect to its underlying stock price $S_{t}$, and can be derived as follows:

From Definition 3.1 and using the valuation formula of the Call option in (2.5.2), we have:

$$
\begin{aligned}
\Delta_{t}^{C} & =\frac{\partial}{\partial S_{t}} V_{t}^{C} \\
& =\Phi\left(d_{1, t}\right)+S_{t} \frac{\partial}{\partial S_{t}} \Phi\left(d_{1, t}\right)-K e^{-r(T-t)} \frac{\partial}{\partial S_{t}} \Phi\left(d_{2, t}\right)
\end{aligned}
$$

Note that, for $k=1,2$

$$
\begin{aligned}
\frac{\partial}{\partial S_{t}} \Phi\left(d_{k}\right) & =\Phi^{\prime}\left(d_{k}\right) \frac{\partial}{\partial S_{t}} d_{k} \\
& =\frac{\phi\left(d_{k}\right)}{S_{t} \sigma \sqrt{T-t}} \\
& =\frac{1}{\sqrt{2 \pi}} e^{-\frac{d_{k}^{2}}{2}} \frac{1}{S_{t} \sigma \sqrt{T-t}}
\end{aligned}
$$


Applying this to the equation of $\Delta_{t}^{C}$ we get

$$
\begin{aligned}
\Delta_{t}^{C}= & \Phi\left(d_{1, t}\right)+S_{t} \frac{1}{\sqrt{2 \pi}} e^{-\frac{d_{1, t}^{2}}{2}} \frac{1}{S_{t} \sigma \sqrt{T-t}} \\
& -K e^{-r(T-t)} \frac{1}{\sqrt{2 \pi}} e^{-\frac{d_{2, t}^{2}}{2}} \frac{1}{S_{t} \sigma \sqrt{T-t}} \\
= & \Phi\left(d_{1, t}\right)+\frac{e^{-\frac{d_{1, t}^{2}}{2}}}{\sqrt{2 \pi} \sigma \sqrt{T-t}}\left[1-\frac{K}{S_{t}} e^{-r(T-t)} e^{-\frac{1}{2}\left(\sigma^{2}(T-t)-2 d_{1, t} \sigma \sqrt{T-t}\right)}\right] .
\end{aligned}
$$

By replacing the formula for $d_{1, t}$ from (2.5.3), the last term becomes 0 , and we get the Delta of a European Call option as:

$$
\Delta_{t}^{C}=\Phi\left(d_{1, t}\right)
$$

By simply looking at the payoff function $\Lambda^{C}$ of the Call, we can observe that the greater the price of the underlying asset at maturity $S_{T}$, the greater the payoff. So the option's value $V_{t}^{C}$ will increase as the price of the underlying asset rises, and will decrease as $S_{t}$ falls. This is an example of a positive correlation between these two financial instruments: the Call option and its underlying. (Note that in the case of a Put option, this correlation is negative). Hence, this correlation is exploited to remove the risk associated with the randomness of the stock price, and thus create the Delta-hedging replicating portfolio $\psi^{\Delta}$, with $\Delta_{t}^{C}=\Phi\left(d_{1, t}\right)$ number of shares held in the portfolio, and $V_{t}^{C}-\Delta_{t}^{C} S_{t}=-K e^{-r(T-t)} \Phi\left(d_{2, t}\right)$ units in the money market account. Then the value of $\psi_{t}^{\Delta}$ for all $0 \leq t \leq T$ is:

$$
\begin{aligned}
V_{t}^{\Delta} & =\psi_{S, t}^{\Delta} S_{t}+\psi_{M, t}^{\Delta} M_{t} \\
& =\Phi\left(d_{1, t}\right) S_{t}-K e^{-r(T-t)} \Phi\left(d_{2, t}\right) \\
& =V_{t}^{C}
\end{aligned}
$$

Most importantly, at expiration $T$, the value of the Delta-hedging portfolio is, $V_{T}^{\Delta}=$ $S_{T}-K$, equal to the payoff of the Call option $\Lambda^{C}$. Thus when the option expires, the 
investor's funds generated by liquidating this portfolio will be sufficient to pay the buyer of the option, in case he exercised his right.

Given this positive correlation between the Call option and its underlying, an option's Delta is impacted by a couple of different factors; its strike price $K$ relative to the share price $S_{t}$, also known as the "moneyness" of the option, and the time until expiration $T-t$. Therefore, the Delta of a Call option is positive, and ranges from 0 to 1 , whereas the Delta of a Put option is negative and ranges from -1 to 0 . Additionally, the Delta of an at-the-money option is around 0.5, and as the option gets further in-the-money, its Delta approaches the value 1 for Calls (and -1 for Puts). Which makes sense because, the deeper in-the-money an option is, the less time value there is on the option, and thus the more it is supposed to behave like the underlying stock.

Also note that, the Deltas of options are additive. Meaning that suppose you hold a portfolio of quantity $w_{i}$ of option $i$, where all options depend on the same asset $S$, then the overall Delta of your whole portfolio is simply the sum over all $i$ of the $i^{\text {th }}$ individual option's Delta multiplied by the corresponding quantity $w_{i} ; \Delta_{\text {Portfolio }}=\sum_{i} \Delta_{i} w_{i}$.

Furthermore, a portfolio is said to be Delta-neutral when it is composed of the right amount of options and/or stocks such that the sum of all the individual Deltas, thus the overall portfolio Delta, is equal to zero.

\subsubsection{Gamma-Hedging}

Delta-hedging is used to reduce the risk of a portfolio inherent to take a position in a financial derivative due to small changes in the underlying stock price. When the volatility of the stock price process is relatively high, the price is more susceptible to have wider variations from the mean. More importantly, we will see in the following section that we are required to apply our hedging strategy in discrete time. It follows that we will be recording the stock's price at discrete points in time. The bigger the interval of time 
between each two subsequent price records, the more it becomes possible to have wider price changes. For this main reason, a more effective risk management technique requires adding a second order hedging to reduce the exposure to risks due to bigger changes in the underlying price.

The Greek letter $\Gamma$ represents a second order hedge parameter that measures the sensitivity of $\Delta$ with respect to changes in the underlying stock price, while all other variables remain unchanged.

Definition 3.2. Mathematically speaking, $\Gamma$ is computed as the second-order derivative of the value of the position (or instrument) to be hedged, $V$, with respect to the price of its underlying asset $S$

$$
\Gamma_{t}=\frac{\partial^{2} V_{t}}{\partial S_{t}^{2}}=\frac{\partial \Delta_{t}}{\partial S_{t}}
$$

Let $\psi^{\Gamma}$ be the Gamma-hedging strategy for the derivative security in question, and denote by $V_{t}^{\Gamma}$ the value of the Gamma-hedging replicating portfolio consisting of that same strategy $\psi^{\Gamma}$.

Then for $V_{t}^{\Gamma}$ to have the same sensitivity to small and bigger changes in the stock price as that of $V_{t}$, we must match both their Deltas and Gammas simultaneously, such that:

$$
\begin{aligned}
\Delta_{t}^{\Gamma} & =\Delta_{t}, \text { and } \\
\Gamma_{t}^{\Gamma} & =\Gamma_{t},
\end{aligned}
$$

where $\Delta_{t}^{\Gamma}$ and $\Gamma_{t}^{\Gamma}$ are the Delta and Gamma of the Gamma-hedging portfolio respectively, and $\Delta_{t}$ and $\Gamma_{t}$ are the Delta and Gamma of the security to be hedged, respectively at any time $t$

Note that since $\Gamma$ is a second order derivative, the Gamma of a portfolio consisting of 
only shares of stock and money market accounts is always zero, given that the value of this portfolio is a linear function of $S_{t}$ and the second derivative of a linear function is zero. Therefore, the Gamma-hedging strategy requires adding a third asset to the replicating portfolio, whose second derivative with respect to $S_{t}$ exists and is different than zero. Without loss of generality, we assume in this thesis that this third asset is a European Call option that is always available on the financial market.

The value of a European Call option $V_{t}^{C}$ for example as given in (2.5.2) is a non-linear function in its underlying stock price $S_{t}$, and hence has a non-zero Gamma.

\subsubsection{Example 2. Gamma of a European Call option}

Under the Black-Scholes model, the Gamma of a European Call option at $0 \leq t \leq T$ is the rate of change of its Delta represented in (3.2.5) with respect to the underlying $S_{t}$, and can be derived as follows:

By combining Definition 3.2.7 and (3.2.5) we have:

$$
\begin{aligned}
\Gamma_{t}^{C} & =\frac{\partial}{\partial S_{t}} \Delta_{t}^{C} \\
& =\frac{\partial}{\partial S_{t}} \Phi\left(d_{1, t}\right) .
\end{aligned}
$$

Then from (3.2.4) we get the Gamma of a European Call option as:

$$
\Gamma_{t}^{C}=\frac{\phi\left(d_{1, t}\right)}{S_{t} \sigma \sqrt{T-t}}
$$

for $0 \leq t \leq T$, where $\phi$ is the standard normal density function, and $d_{1, t}$ is as given in $(2.5 .3)$

In general, $\Gamma$ is the lowest for deep in-the-money and out-of-the-money options, and 
highest as the option gets near the money. For long options, $\Gamma$ takes a positive value, and for short options it takes a negative value.

As a result, the replicating portfolio is now composed of $\psi_{S, t}^{\Gamma}$ shares of the underlying $S, \psi_{C, t}^{\Gamma}$ Call options on the same underlying stock $S$, and $\psi_{M, t}^{\Gamma}$ units in the money market account $M$ at each time $t$.

Thus we can write:

$$
\psi^{\Gamma}=\left\{\psi_{S}^{\Gamma}, \psi_{C}^{\Gamma}, \psi_{M}^{\Gamma}\right\}
$$

and

$$
V_{t}^{\Gamma}=\psi_{S, t}^{\Gamma} S_{t}+\psi_{C, t}^{\Gamma} V_{t}^{C}+\psi_{M, t}^{\Gamma} M_{t}
$$

Then the $\Delta$ and $\Gamma$ of the Gamma-hedging portfolio are given as follows:

$$
\begin{aligned}
\Delta_{t}^{\Gamma} & =\frac{\partial}{\partial S_{t}} V_{t}^{\Gamma} \\
& =\psi_{S, t}^{\Gamma}+\psi_{C, t}^{\Gamma} \Delta_{t}^{C} \\
& =\psi_{S, t}^{\Gamma}+\psi_{C, t}^{\Gamma} \Phi\left(d_{1, t}\right),
\end{aligned}
$$

and

$$
\begin{aligned}
\Gamma_{t}^{\Gamma} & =\frac{\partial}{\partial S_{t}} \Delta_{t}^{\Gamma} \\
& =\psi_{C, t}^{\Gamma} \Gamma_{t}^{C} \\
& =\psi_{C, t}^{\Gamma} \frac{\phi\left(d_{1, t}\right)}{S_{t} \sigma \sqrt{T-t}} .
\end{aligned}
$$

Now to get the proportions of the hedging portfolio $\psi_{t}^{\Gamma}$ at any time $0 \leq t \leq T$ we start by matching the Gammas, $\Gamma_{t}^{\Gamma}$ and $\Gamma_{t}$, and combining (3.2.8) and (3.2.12) to get 
$\psi_{C, t}^{\Gamma} \Gamma_{t}^{C}=\Gamma_{t}$. Thus the number of Calls in the Gamma-hedging portfolio is given by:

$$
\psi_{C, t}^{\Gamma}=\frac{\Gamma_{t}}{\Gamma_{t}^{C}}
$$

By adding $\psi_{C, t}^{\Gamma}$ number of Calls to the hedging portfolio, its Delta changes since it is affected by the Deltas of the Call options $\Delta_{t}^{C}$. Therefore, we need to match the Deltas, $\Delta_{t}^{\Gamma}$ and $\Delta_{t}$, by combining (3.2.8) and (3.2.11) to get $\psi_{S, t}^{\Gamma}+\psi_{C, t}^{\Gamma} \Delta_{t}^{C}=\Delta_{t}$. Thus after fixing the number of Calls $\psi_{C, t}^{\Gamma}$ at each time $0 \leq t \leq T$, the number of shares in the replicating portfolio is given by:

$$
\psi_{S, t}^{\Gamma}=\Delta_{t}-\psi_{C, t}^{\Gamma} \Delta_{t}^{C}
$$

Finally, the difference between the value of the derivative to be hedged $V_{t}$ and the amounts of money invested in the stocks and Calls gives the amount to be invested in the money market account;

$$
\psi_{M, t}^{\Gamma} M_{t}=V_{t}-\left(\Delta_{t}-\psi_{C, t}^{\Gamma} \Delta_{t}^{C}\right) S_{t}-\left(\frac{\Gamma_{t}}{\Gamma_{t}^{C}}\right) V_{t}^{C}
$$

thus keeping the value of the replicating portfolio always equal to that of the derivative to be hedged.

Assume that we take a short position in the derivative security $V_{t}$ and hedge it by taking a long position in the replicating portfolio $\psi^{\Gamma}$ constructed using the Greeks.

By the arbitrage-free risk neutral pricing principle, our overall position $\Pi_{t}^{\Gamma}=V_{t}^{\Gamma}-V_{t}$ is risk-free and earns the risk-free rate of return $r$. Moreover, given that this replicating portfolio is constructed by matching both Deltas and Gammas, the Delta and Gamma of our overall position are both zero, and we say the the overall portfolio $\Pi$ is Delta and Gamma neutral.

$$
\begin{gathered}
\frac{\partial \Pi_{t}^{\Gamma}}{\partial S_{t}}=\Delta_{t}^{\Pi}=\Delta_{t}^{\Gamma}-\Delta_{t}=0, \\
\frac{\partial^{2} \Pi_{t}^{\Gamma}}{\partial S_{t}^{2}}=\Gamma_{t}^{\Pi}=\Gamma_{t}^{\Gamma}-\Gamma_{t}=0 .
\end{gathered}
$$

The risks inherent to create Delta-Gamma neutral portfolios arise when the price of 
the underlying moves strongly either up or down, in one direction only. In other words, when the stock price does not fluctuate around its mean.

\subsection{Hedging in Discrete Time}

It is important to note that both $\Delta_{t}^{\Pi}$ and $\Gamma_{t}^{\Pi}$ are functions of time $t$ and vary constantly as the price of the underlying $S_{t}$ changes. Therefore, with the passage of time, to maintain Delta and Gamma neutrality of $\Pi$ an investor must constantly adjust his positions by trading the stocks and options during the lifetime of the derivative instrument. This is known as dynamic hedging.

Recall that a perfect hedging strategy totally eliminates the risk in the initial position, otherwise, it is said to be partial. In theory, hedging must be done in a continuous manner to obtain a perfect hedge. However in reality hedging must be done in discrete time. Then how many times, per a given period, an investor has to re-balance his hedging strategy?

\subsubsection{Hedging Frequency}

To address this question, it seems logical to point out that an investor must study the impact of the re-balancing frequency. On the one hand, it is clear that the more we hedge, that is the more frequent the re-balancing is done, the closer we are to the perfect complete hedging conditions. On the other hand, we must criticize the assumption of efficient markets where we considered that the market is frictionless and very liquid, by admitting that transaction costs indeed exist, and represent an expense to the issuer. The existence of the bid and ask spread on almost all transactions renders the dynamic business of Delta - and even more that of Gamma - hedging somewhat expensive, and forces the investor to reduce the frequency of his re-balancing. Therefore, the lower the transaction costs in the market for the underlying, the more frequent the re-balancing could be done (or afforded).

Different approaches had been proposed to account for the presence of proportional 
and fixed transaction costs in pricing and hedging financial derivatives. The most common ones available in the literature include Clewlow and Hodges (1997), Monoyios (2004), Zakamouline (2006), Lai and Lim (2009), and many more, where they present a variety of optimal hedging strategies, such that the maximization of expected utility of terminal wealth, or minimum cost super-replication strategies, etc... Since most of these strategies are path dependent and require computationally expensive dynamic programming algorithms, we will not be looking into the existence of transactions costs in our thesis.

Apart from the impact of - or the limitation set by - the transaction costs, the rebalancing frequency could also be somehow determined by looking at the portfolio's Delta and Gamma values. Since Gamma measures the speed of change of Delta, when Gamma is small, Delta changes slowly when the asset price changes. Consequently, this portfolio could be re-balanced infrequently. Whereas if Gamma is relatively big, then Delta changes quickly when the asset price changes, and it follows that this portfolio would require more frequent re-balancing.

\subsubsection{Hedging Errors}

In this thesis, we stick with the assumption of frictionless markets to eliminate the effect of transaction costs on portfolio re-balancing in a discrete manner, and the only time we break the assumption of complete markets is through discrete hedging. However, apart from that, the process of applying a discrete-time trading strategy based on a continuous-time model also brings in another deviation from the theory to the application. In reality, basis risk always exists. Basis risk refers to the risk that a position in a derivative and its hedging portfolio do not move in opposite directions as desired. In fact, the word basis refers to the discrepancy, and can be measured by what we call the Hedging Errors. Hedging errors represent costs arising from the basis risk of the actual discrete versus the theoretical continuous hedge.

In practice, re-balancing could be done monthly, weekly, or even daily for example. 
However, no matter how frequent it is, hedging errors would still occur and are inevitable. Since re-balancing has to be done periodically, the hedging strategy using the Greeks applied in a discrete manner only allows the investor to keep the value of his replicating portfolio $V_{t}^{\psi}$ close - but not equal - to the price of the derivative being hedged during each adjustment period. Therefore, these periodic adjustments force the value of the replicating portfolio to deviate from that of the derivative. We assume that re-balancing is done $m$ times per year at predefined (non-random) and equally spaced discrete times $t_{i}, i=0,1, \ldots, m$, where $t_{i}-t_{i-1}=\frac{1}{m}$. Thus hedging errors arise as the investor changes the proportions of his portfolio to keep the Greeks matched at discrete time intervals $t_{1}, t_{2}, \ldots, t_{m}$

By definition, hedging errors are the difference between the actual accumulated value of the replicating portfolio and the price of the derivative security being hedged, at the end of each of the discrete hedging intervals, right before re-balancing occurs.

Define $H E_{t}$ to be the value of the hedging error at time $t$, and let $V_{t^{-}}^{\psi}$ be the accumulated portfolio value at time $t$ right before re-balancing, then the following holds:

$$
H E_{t}=V_{t^{-}}^{\psi}-V_{t}
$$

Based of this formula, the hedging error can be interpreted as what we own (the portfolio accumulated value) less what we owe (the security sold short). Therefore, when negative it represents a cost incurred, or an amount by which we are forced to re-invest in the replicating portfolio, and when positive, it represents a surplus that can be withdrawn from the portfolio.

In general, the present value of the Total Hedging Errors is the sum of all periodic errors discounted back to time 0 ,

$$
T H E_{0}=\sum_{t=0}^{T-1} \sum_{i=1}^{m} e^{-\left(t+\frac{i}{m}\right) r} H E_{t+\frac{i}{m}}
$$


$T H E_{0}$ can be thought of as the added cost of the hedging strategy at time 0 due to discretization, and thus can be used to assess its effectiveness and compare its performance relative to other hedging strategies. Intuitively, a better hedging strategy will incur less hedging errors, as well as a more frequent re-balancing.

\subsubsection{Discretization of the Stock Price Process}

Later in this thesis we introduce a numerical example for the hedging of a special derivative security, the Annual Compound Rathet Equity-Indexed Annuity. This requires generating sample paths of stock prices under the Black-Scholes model. Therefore we shall first introduce a model that discretizes the continuous-time model of the stock price process presented in Section 2.4, particularly in (2.4.2).

When simulating stock price paths, it is more common and useful to generate their periodic returns instead. The dynamics of the stock price process can be expressed in terms of the distribution of their log-returns. In fact, (2.4.1) can be written in this way:

$$
\frac{d S(t)}{S(t)}=\mu d t+\sigma d B(t)
$$

where $\frac{d S(t)}{S(t)}$ in this equation represents the relative or percentage increment in the stock price $S(t)$, or equivalently, the instantaneous rate of return on the stock, during an instant of time $d t$. The constant $\mu$ represents the expected instantaneous rate of return, and $\sigma$ represents the standard deviation of the instantaneous rate of return.

A simple manipulation of (2.4.2) by letting $t=t_{i}$, and $t_{i-1}$ gives:

$$
S\left(t_{i}\right)=S(0) \exp \left[\left(\mu-\sigma^{2} / 2\right)\left(t_{i}\right)+\sigma B\left(t_{i}\right)\right],
$$

and

$$
S\left(t_{i-1}\right)=S(0) \exp \left[\left(\mu-\sigma^{2} / 2\right)\left(t_{i-1}\right)+\sigma B\left(t_{i-1}\right)\right] .
$$


Dividing the former by the latter we get:

$$
\frac{S\left(t_{i}\right)}{S\left(t_{i-1}\right)}=\exp \left[\left(\mu-\sigma^{2} / 2\right)\left(t_{i}-t_{i-1}\right)+\sigma\left(B\left(t_{i}\right)-B\left(t_{i-1}\right)\right)\right]
$$

By taking the natural logarithm of the accumulation factor and replacing $t_{i}-t_{i-1}$ by $\frac{1}{m}$ we get:

$$
\ln \frac{S\left(t_{i}\right)}{S\left(t_{i-1}\right)}=\left(\mu-\sigma^{2} / 2\right)\left(\frac{1}{m}\right)+\sigma\left(B\left(t_{i}\right)-B\left(t_{i-1}\right)\right)
$$

Recall from Section 2.4.1 that for $t_{i-1}<t_{i}<t_{i+1}, \quad B\left(t_{i+1}\right)-B\left(t_{i}\right) \sim N\left(0, t_{i+1}-t_{i}\right) \equiv$ $N\left(0, \frac{1}{m}\right)$ is independent of $B\left(t_{i}\right)-B\left(t_{i-1}\right) \sim N\left(0, \frac{1}{m}\right)$. Then we have

$$
\ln \frac{S\left(t_{i}\right)}{S\left(t_{i-1}\right)}=\left(\mu-\sigma^{2} / 2\right)\left(\frac{1}{m}\right)+\sigma \sqrt{\frac{1}{m}} N(0,1)
$$

It follows that the natural logarithm of periodic accumulation factors have a normal distribution with mean $\left(\mu-\sigma^{2} / 2\right)\left(\frac{1}{m}\right)$ and variance $\frac{\sigma^{2}}{m}$, that is

$$
\ln \frac{S\left(t_{i}\right)}{S\left(t_{i-1}\right)} \sim N\left(\left(\mu-\sigma^{2} / 2\right)\left(\frac{1}{m}\right), \frac{\sigma^{2}}{m}\right) .
$$

Equivalently, successive (non-overlapping) periodic accumulation factors can be sampled directly from the log-normal distribution with the same parameters, assuming that stock prices are observed at the beginning of each period;

$$
\frac{S\left(t_{i}\right)}{S\left(t_{i-1}\right)} \sim L N\left(\left(\mu-\sigma^{2} / 2\right)\left(\frac{1}{m}\right), \frac{\sigma^{2}}{m}\right) .
$$




\section{Chapter 4}

\section{Pricing Equity-Indexed Annuities}

This chapter presents the pricing at time zero of one the most popular type of EIAs presented in Section 1.4; the Annual Compound Ratchet EIA contract, and derives a closed-form expression for the value of this contract at any fractional time $0 \leq t \leq T$ from inception till maturity. The purpose of this valuation formula is to be able to hedge this contract using the Greeks presented in Chapter 3.

\subsection{Time-0 Price of Annual Compound Ratchet EIA}

To begin with, recall that EIA contracts do not invest the initial capital directly in the underlying equity, therefore buyers of such contracts will not be receiving any dividends earned on the index. This permits us to exclude the dividends from our model.

Without loss of generality, we assume throughout this thesis that the initial capital invested (denoted by $I C$ in Section 1.4) is one monetary unit in order to simplify our expressions. Therefore, it suffices to multiply them by the appropriate initial investment to get the desired results.

We begin by pricing the Annual Compound Ratchet EIA under the Black-Scholes model assumptions presented in Chapter 2. The payoff structure $\Lambda^{C R}$ at maturity of the 
contract $T$ is given by:

$$
\Lambda^{C R}=\prod_{t=1}^{T} 1+\min \left(\max \left(\alpha\left(R_{t}-1\right), f\right), c\right)
$$

where $f$ is the local floor rate, $c$ is the local cap rate, $\alpha$ participation rate in the index return with $0 \leq f, c, \alpha \leq 1$. $R_{t}$ is the yearly return credited from the underlying index during year $t, 1 \leq t \leq T$ under the Term-End Point design, and used without averaging, such that

$$
R_{t}=\frac{S(t)}{S(t-1)}
$$

By ignoring mortality risk, the valuation of an EIA contract simplifies to the valuation of a pure financial security. Therefore, using the risk-neutral valuation under the martingale measure $\mathbb{Q}$, we can obtain a closed-form solution for the price at time zero of the Annual Compound Ratchet EIA.

Proposition 4.1. Under the Black-Scholes framework, the price at time zero $V_{0}^{C R}$ of a $T$-year Compound Annual Ratchet EIA contract with payoff $\Lambda^{C R}$ given in (4.1.1) has the following expression:

$V_{0}^{C R}=e^{-T r}\left[(1+f) \Phi\left(-l_{2}\right)+(1-\alpha)\left(\Phi\left(l_{2}\right)-\Phi\left(l_{4}\right)\right)+\alpha e^{r}\left(\Phi\left(l_{1}\right)-\Phi\left(l_{3}\right)\right)+(1+c) \Phi\left(l_{4}\right)\right]^{T}$ where $l_{1}=\frac{-\ln \left(1+\frac{f}{\alpha}\right)+r+\frac{\sigma^{2}}{2}}{\sigma}, l_{2}=l_{1}-\sigma=-\frac{\ln \left(1+\frac{f}{\alpha}\right)-\left(r-\frac{\sigma^{2}}{2}\right)}{\sigma}, l_{3}=\frac{-\ln \left(1+\frac{c}{\alpha}\right)+r+\frac{\sigma^{2}}{2}}{\sigma}$, and $l_{4}=l_{3}-\sigma=-\frac{\ln \left(1+\frac{c}{\alpha}\right)-\left(r-\frac{\sigma^{2}}{2}\right)}{\sigma}$.

Proof. The price at time zero $V_{0}^{C R}$ of the Annual Compound Ratchet EIA is given by the expectation under the risk-neutral measure $\mathbb{Q}$, denoted by $E^{\mathbb{Q}}[$.$] , of its payoff \Lambda^{C R}$ at time 
$T$, discounted back to time 0 at the constant risk-free rate of return $r$, that is

$$
\begin{aligned}
V_{0}^{C R} & =\mathbb{E}^{\mathbb{Q}}\left[e^{-T r} \Lambda^{C R}\right] \\
& =e^{-T r} \mathbb{E}^{\mathbb{Q}}\left[\prod_{t=1}^{T} 1+\min \left(\max \left(\alpha\left(R_{t}-1\right), f\right), c\right)\right] .
\end{aligned}
$$

Under the Black-Scholes model, the return on the stock price process is Markovian, then the yearly index returns $R_{t}$ are independent and identically distributed. Therefore, (4.1.4) becomes:

$$
\begin{aligned}
& e^{-T r} \prod_{t=1}^{T} \mathbb{E}^{\mathbb{Q}}\left[1+\min \left(\max \left(\alpha\left(R_{t}-1\right), f\right), c\right)\right] \\
= & e^{-T r}\left\{\mathbb{E}^{\mathbb{Q}}\left[1+\min \left(\max \left(\alpha\left(R_{t}-1\right), f\right), c\right)\right]\right\}^{T} \\
= & e^{-T r}\left\{\mathbb{E}^{\mathbb{Q}}\left[\mathcal{R}_{t}\right]\right\}^{T} .
\end{aligned}
$$

Consequently, finding the time-0 price $V_{0}^{C R}$ simplifies to solving a single risk neutral expectation, $\mathbb{E}^{\mathbb{Q}}\left[\mathcal{R}_{t}\right]$, where $\mathcal{R}_{t}$ denotes the yearly credited return,

$$
\mathcal{R}_{t}=1+\min \left(\max \left(\alpha\left(R_{t}-1\right), f\right), c\right) .
$$

Depending on the performance of the underlying stock index reflected by $R_{t}$, the actual credited return $\mathcal{R}_{t}$ during each year can take one of three possible values:

$$
\mathcal{R}_{t}=\left\{\begin{array}{llr}
1+f & \text { when } & R_{t}<K_{1} \\
1-\alpha+\alpha R_{t} & \text { when } & K_{1}<R_{t} \leq K_{2} \\
1+c & \text { when } & R_{t}>K_{2}
\end{array}\right.
$$

Where $K_{1}=1+\frac{f}{\alpha}$ and $K_{2}=1+\frac{c}{\alpha}$. 
Recall from (3.3.3) that successive non-overlapping periodic accumulation factors $\frac{S\left(t_{i}\right)}{S\left(t_{i-1}\right)}$ are independent and identically log-normally distributed with parameters $\left(\mu-\sigma^{2} / 2\right)\left(\frac{1}{m}\right)$ and $\frac{\sigma^{2}}{m}$. Then under the risk-neutral distribution, the yearly returns $R_{t}=\frac{S(t)}{S(t-1)}$ are independent and identically log-normally distributed with parameters $r-\sigma^{2} / 2$ and $\sigma^{2}$, (with $m=1$ period per year), with probability density function $f_{R_{t}}(x)=\frac{1}{x \sqrt{2 \pi} \sigma} \exp \left\{-\frac{\left[\ln x-\left(r-\frac{\sigma^{2}}{2}\right)\right]^{2}}{2 \sigma^{2}}\right\}$, and cumulative distribution function $F_{R_{t}}(x)=\Phi\left(\frac{\ln x-\left(r-\frac{\sigma^{2}}{2}\right)}{\sigma}\right)$.

It follows that taking the risk neutral expectation of $\mathcal{R}_{t}$ we get:

$$
\mathbb{E}^{\mathbb{Q}}\left[\mathcal{R}_{t}\right]=\int_{0}^{K_{1}}(1+f) f_{R_{t}}(x) d x+\int_{K_{1}}^{K_{2}}(1-\alpha+\alpha x) f_{R_{t}}(x) d x+\int_{K_{2}}^{\infty}(1+c) f_{R_{t}}(x) d x
$$

Focusing on the first integral of the right hand side of (4.1.7), we have:

$$
\begin{aligned}
\int_{0}^{K_{1}}(1+f) f_{R_{t}}(x) d x & =(1+f) F_{R_{t}}\left(K_{1}\right) \\
& =(1+f) \Phi\left(\frac{\ln K_{1}-\left(r-\frac{\sigma^{2}}{2}\right)}{\sigma}\right) \\
& =(1+f) \Phi\left(-l_{2}\right)
\end{aligned}
$$

where $l_{2}$ is given in Proposition 4.1.

The second integral of the right hand side of (4.1.7) gives: 


$$
\begin{aligned}
& \int_{K_{1}}^{K_{2}}(1-\alpha+\alpha x) f_{R_{t}}(x) d x \\
& =\int_{K_{1}}^{K_{2}}(1-\alpha) f_{R_{t}}(x) d x+\int_{K_{1}}^{K_{2}}(\alpha x) f_{R_{t}}(x) d x \\
& \left.\left.=(1-\alpha)\left[F_{R_{t}}\left(K_{2}\right)-F_{R_{t}}\left(K_{1}\right)\right]+\alpha \int_{K_{1}}^{K_{2}} \frac{1}{x \sqrt{2 \pi} \sigma} \exp \left\{-\frac{\ln ^{2}}{2}\right)\right]^{2}\right) \\
& =(1-\alpha)\left[\Phi\left(\frac{\ln K_{2}-\left(r-\frac{\sigma^{2}}{2}\right)}{\sigma}\right)-\left(\frac{\ln K_{1}-\left(r-\frac{\sigma^{2}}{2}\right)}{\sigma}\right)\right] \\
& \quad+\alpha \int_{K_{1}}^{K_{2}} \frac{1}{\sqrt{2 \pi} \sigma} \exp \left\{-\frac{\left[\ln x-\left(r-\frac{\sigma^{2}}{2}\right)\right]^{2}}{2 \sigma^{2}}\right\} d x .
\end{aligned}
$$

By applying the identity $\Phi(A)-\Phi(B)=\Phi(-B)-\Phi(-A)$ on the first term, and the change of variable $y=\ln x$ with $d x=e^{y} d y$ on the second term, we get:

$$
\begin{aligned}
& \int_{K_{1}}^{K_{2}}(1-\alpha+\alpha x) f_{R_{t}}(x) d x \\
& =(1-\alpha)\left[\Phi\left(-\frac{\ln K_{1}-\left(r-\frac{\sigma^{2}}{2}\right)}{\sigma}\right)-\Phi\left(-\frac{\ln K_{2}-\left(r-\frac{\sigma^{2}}{2}\right)}{\sigma}\right)\right] \\
& \quad+\alpha \int_{\ln K_{1}}^{\ln K_{2}} \frac{1}{\sqrt{2 \pi} \sigma} \exp \left\{-\frac{\left[y-\left(r-\frac{\sigma^{2}}{2}\right)\right]^{2}}{2 \sigma^{2}}\right\} \exp \{y\} d y
\end{aligned}
$$




$$
\begin{aligned}
& =(1-\alpha)\left[\Phi\left(l_{2}\right)-\Phi\left(l_{4}\right)\right] \\
& +\alpha \int_{\ln K_{1}}^{\ln K_{2}} \frac{1}{\sqrt{2 \pi} \sigma} \exp \left\{-\frac{\left[y-\left(r+\frac{\sigma^{2}}{2}\right)\right]^{2}}{2 \sigma^{2}}\right\} \exp \left\{\frac{\left[r+\frac{\sigma^{2}}{2}\right]^{2}-\left[r-\frac{\sigma^{2}}{2}\right]^{2}}{2 \sigma^{2}}\right\} d y \\
& =(1-\alpha)\left[\Phi\left(l_{2}\right)-\Phi\left(l_{4}\right)\right] \\
& +\alpha e^{r} \int_{\ln K_{1}}^{\ln K_{2}} \frac{1}{\sqrt{2 \pi} \sigma} \exp \left\{-\frac{\left[y-\left(r+\frac{\sigma^{2}}{2}\right)\right]^{2}}{2 \sigma^{2}}\right\} d y \\
& =(1-\alpha)\left[\Phi\left(l_{2}\right)-\Phi\left(l_{4}\right)\right] \\
& +\alpha e^{r}\left[\Phi\left(\frac{\ln K_{2}-\left(r+\frac{\sigma^{2}}{2}\right)}{\sigma}\right)-\Phi\left(\frac{\ln K_{1}-\left(r+\frac{\sigma^{2}}{2}\right)}{\sigma}\right)\right] \\
& =(1-\alpha)\left[\Phi\left(l_{2}\right)-\Phi\left(l_{4}\right)\right] \\
& +\alpha e^{r}\left[\Phi\left(-\frac{\ln K_{1}-\left(r+\frac{\sigma^{2}}{2}\right)}{\sigma}\right)-\Phi\left(-\frac{\ln K_{2}-\left(r+\frac{\sigma^{2}}{2}\right)}{\sigma}\right)\right] \\
& =(1-\alpha)\left[\Phi\left(l_{2}\right)-\Phi\left(l_{4}\right)\right] \\
& +\alpha e^{r}\left[\Phi\left(\frac{-\ln K_{1}+\left(r+\frac{\sigma^{2}}{2}\right)}{\sigma}\right)-\Phi\left(\frac{-\ln K_{2}+\left(r+\frac{\sigma^{2}}{2}\right)}{\sigma}\right)\right] \\
& =(1-\alpha)\left[\Phi\left(l_{2}\right)-\Phi\left(l_{4}\right)\right]+\alpha e^{r}\left[\Phi\left(l_{1}\right)-\Phi\left(l_{3}\right)\right],
\end{aligned}
$$

where $l_{1}, l_{2}, l_{3}$, and $l_{4}$ are given in Proposition 4.1. 
Then the third integral of the right hand side of (4.1.7) gives:

$$
\begin{aligned}
\int_{K_{2}}^{\infty}(1+c) f_{R_{t}}(x) d x & =(1+c)\left[1-F_{R_{t}}\left(K_{2}\right)\right] \\
& =(1+c)\left[1-\Phi\left(\frac{\ln K_{2}-\left(r-\frac{\sigma^{2}}{2}\right)}{\sigma}\right)\right] \\
& =(1+c) \Phi\left(-\frac{\ln K_{2}-\left(r-\frac{\sigma^{2}}{2}\right)}{\sigma}\right) \\
& =(1+c) \Phi\left(l_{4}\right)
\end{aligned}
$$

where $l_{4}$ is given in Proposition 4.1.

Consequently by combining the results of the integrals from (4.1.8), (4.1.9) and (4.1.10), then (4.1.7) becomes:

$$
\mathbb{E}^{\mathbb{Q}}\left[\mathcal{R}_{t}\right]=(1+f) \Phi\left(-l_{2}\right)+(1-\alpha)\left[\Phi\left(l_{2}\right)-\Phi\left(l_{4}\right)\right]+\alpha e^{r}\left[\Phi\left(l_{1}\right)-\Phi\left(l_{3}\right)\right]+(1+c) \Phi\left(l_{4}\right),
$$

and we finally get the time- 0 price in the form

$$
\begin{aligned}
V_{0}^{C R} & =e^{-T r}\left\{\mathbb{E}^{\mathbb{Q}}\left[\mathcal{R}_{t}\right]\right\}^{T} \\
& =e^{-T r}\left\{(1+f) \Phi\left(-l_{2}\right)+(1-\alpha)\left[\Phi\left(l_{2}\right)-\Phi\left(l_{4}\right)\right]+\alpha e^{r}\left[\Phi\left(l_{1}\right)-\Phi\left(l_{3}\right)\right]+(1+c) \Phi\left(l_{4}\right)\right\}^{T}
\end{aligned}
$$

where we have proved Proposition 4.1. 


\subsection{Time- $t$ Price of Annual Compound Ratchet EIA}

The analytic solution for the price at inception of an EIA contract is important sufficient when hedging the contract statistically. However, for the purpose of applying the hedging strategy dynamically, we have to find also a closed-form formula for the value of the Annual Compound Ratchet EIA at any fractional time $t$ between inception and maturity. Denote by $V_{t}^{C R}$ the price of this contract at time $t$, for $0 \leq t \leq T$, with payoff $\Lambda^{C R}$. $V_{t}^{C R}$ will be conditional on the information known up to time $t$, summarized by the filtration $\mathcal{F}_{t}$.

Proposition 4.2. Under the Black-Scholes framework, $V_{t}^{C R}$ is given by:

$$
\begin{aligned}
V_{t}^{C R}= & e^{-(T-t) r} \prod_{i=1}^{t^{*}} \mathcal{R}_{i} \times\left[(1+f) \Phi\left(-h_{2}\right)+(1-\alpha)\left[\Phi\left(h_{2}\right)-\Phi\left(h_{4}\right)\right]\right. \\
& \left.+\alpha \frac{S_{t}}{S_{t^{*}}} e^{\left(t^{*}+1-t\right) r}\left[\Phi\left(h_{1}\right)-\Phi\left(h_{3}\right)\right]+(1+c) \Phi\left(h_{4}\right)\right] \\
\times & {\left[(1+f) \Phi\left(-l_{2}\right)+(1-\alpha)\left[\Phi\left(l_{2}\right)-\Phi\left(l_{4}\right)\right]+\alpha e^{r}\left[\Phi\left(l_{1}\right)-\Phi\left(l_{3}\right)\right]\right.} \\
& \left.+(1+c) \Phi\left(l_{4}\right)\right]^{T-t^{*}-1}
\end{aligned}
$$

where $\mathcal{R}_{i}$ is given by (4.1.5), $l_{1}, l_{2}, l_{3}$ and $l_{3}$ are given by Proposition $4.1, t^{*}=\lfloor t\rfloor$ is the

greatest integer smaller than $t$, and $h_{1}, h_{2}, h_{3}$ and $h_{4}$ are given by $h_{1}=\frac{-\ln \left(\frac{S_{t^{*}}}{S_{t}}\left(1+\frac{f}{\alpha}\right)\right)+\left(t^{*}+1-t\right)\left(r+\frac{\sigma^{2}}{2}\right)}{\sigma \sqrt{t^{*}+1-t}}$,

$h_{2}=-\frac{\ln \left(\frac{S_{t^{*}}}{S_{t}}\left(1+\frac{f}{\alpha}\right)\right)-\left(t^{*}+1-t\right)\left(r-\frac{\sigma^{2}}{2}\right)}{\sigma \sqrt{t^{*}+1-t}}, h_{3}=\frac{-\ln \left(\frac{S_{t^{*}}}{S_{t}}\left(1+\frac{c}{\alpha}\right)\right)+\left(t^{*}+1-t\right)\left(r+\frac{\sigma^{2}}{2}\right)}{\sigma \sqrt{t^{*}+1-t}}$, and

$h_{4}=-\frac{\ln \left(\frac{S_{t^{*}}}{S_{t}}\left(1+\frac{c}{\alpha}\right)\right)-\left(t^{*}+1-t\right)\left(r-\frac{\sigma^{2}}{2}\right)}{\sigma \sqrt{t^{*}+1-t}}$, with $h_{2}=h_{1}-\sigma \sqrt{t^{*}+1-t}$ and

$h_{4}=h_{3}-\sigma \sqrt{t^{*}+1-t}$.

Proof. Given that we stand at a certain time $t$, where $t^{*} \leq t<t^{*}+1$, the payoff structure of the Annual Compound Ratchet can be seen as the accumulation of each year's credited 
return from time zero until maturity $T$;

$$
\begin{aligned}
\Lambda^{C R} & =\prod_{t=1}^{T} 1+\min \left(\max \left(\alpha\left(R_{t}-1\right), f\right), c\right) \\
& =\prod_{t=1}^{T} \mathcal{R}_{t} \\
& =\mathcal{R}_{1} \times \ldots \times \mathcal{R}_{t^{*}-1} \times \mathcal{R}_{t^{*}} \times \mathcal{R}_{t^{*}+1} \times \ldots \times \mathcal{R}_{T} .
\end{aligned}
$$

The filtration $\mathcal{F}_{t}$ contains all the information on the price process $S$ of the underlying index from time 0 to $t$, and thus the past realized yearly index returns $R_{t}$ up to time $t^{*}$; $R_{1}, R_{2}, \ldots, R_{t^{*}}$, as well as the stock price $S_{t}$, are known. Consequently, the actual credited and accumulated returns $\mathcal{R}_{t}$ - after accounting for $\alpha, f$, and $c$ - are also known for all the past years. Thus, the unknown terms in (4.2.2) are the current year's credited return, $\mathcal{R}_{t^{*}+1}$, and that of the future years left until maturity $\mathcal{R}_{t^{*}+2}, \ldots, \mathcal{R}_{T}$.

It follows that the price $V_{t}^{C R}$ of the EIA contract at time $t$ given the filtration $\mathcal{F}_{t}$, is given by:

$$
\begin{aligned}
V_{t}^{C R} & =\mathbb{E}^{\mathbb{Q}}\left[e^{-(T-t) r} \Lambda^{C R} \mid \mathcal{F}_{t}\right] \\
& =\mathbb{E}^{\mathbb{Q}}\left[e^{-(T-t) r} \prod_{i=1}^{T} \mathcal{R}_{i} \mid \mathcal{F}_{t}\right] \\
& =e^{-(T-t) r} \mathbb{E}^{\mathbb{Q}}\left[\mathcal{R}_{1} \times \ldots \times \mathcal{R}_{t^{*}} \times \mathcal{R}_{t^{*}+1} \times \mathcal{R}_{t^{*}+2} \times \ldots \times \mathcal{R}_{T} \mid \mathcal{F}_{t}\right] .
\end{aligned}
$$

By taking outside what is known and using the fact that yearly index returns are independent and identically distributed we get:

$$
\begin{aligned}
V_{t}^{C R} & =e^{-(T-t) r}\left(\mathcal{R}_{1} \times \ldots \times \mathcal{R}_{t^{*}}\right) \mathbb{E}^{\mathbb{Q}}\left[\mathcal{R}_{t^{*}+1} \times \mathcal{R}_{t^{*}+2} \times \ldots \times \mathcal{R}_{T} \mid \mathcal{F}_{t}\right] \\
& =e^{-(T-t) r}\left(\mathcal{R}_{1} \times \ldots \times \mathcal{R}_{t^{*}}\right) \mathbb{E}^{\mathbb{Q}}\left[\mathcal{R}_{t^{*}+1} \mid \mathcal{F}_{t}\right] \mathbb{E}^{\mathbb{Q}}\left[\mathcal{R}_{t^{*}+2} \times \ldots \times \mathcal{R}_{T} \mid \mathcal{F}_{t}\right] \\
& =e^{-(T-t) r} \prod_{i=1}^{t^{*}}\left(\mathcal{R}_{i}\right) \mathbb{E}^{\mathbb{Q}}\left[\mathcal{R}_{t^{*}+1} \mid \mathcal{F}_{t}\right] \prod_{i=t^{*}+2}^{T} \mathbb{E}^{\mathbb{Q}}\left[\mathcal{R}_{i} \mid \mathcal{F}_{t}\right] \\
& =e^{-(T-t) r} \prod_{i=1}^{t^{*}}\left(\mathcal{R}_{i}\right) \times \mathbb{E}^{\mathbb{Q}}\left[\mathcal{R}_{t^{*}+1} \mid \mathcal{F}_{t}\right] \times\left(\mathbb{E}^{\mathbb{Q}}\left[\mathcal{R}_{i}\right]\right)^{T-t^{*}-1}
\end{aligned}
$$


Given the stock's path, the first term is given by (4.1.6). The third term will be replaced by (4.1.11) raised to the power $T-t^{*}-1$. The unknown part is the middle term $\mathbb{E}^{\mathbb{Q}}\left[\mathcal{R}_{t^{*}+1} \mid \mathcal{F}_{t}\right]$; the risk neutral expectation of the current year's credited return. The reason behind isolating it is because we have partial information about that year's index return by knowing the current stock price $S_{t}$. So from (4.1.2) we are able to split $R_{t^{*}+1}$ into known and unknown parts:

$$
\begin{aligned}
R_{t^{*}+1} & =\frac{S_{t^{*}+1}}{S_{t^{*}}} \\
& =\frac{S_{t}}{S_{t^{*}}} \times \frac{S_{t^{*}+1}}{S_{t}} \\
& =\frac{S_{t}}{S_{t^{*}}} \times R^{*},
\end{aligned}
$$

and we have

$$
\begin{aligned}
\mathcal{R}_{t^{*}+1} & =1+\min \left(\max \left(\alpha\left(R_{t^{*}+1}-1\right), f\right), c\right) \\
& =1+\min \left(\max \left(\alpha\left(\frac{S_{t}}{S_{t^{*}}} R^{*}-1\right), f\right), c\right),
\end{aligned}
$$

where $\frac{S_{t}}{S_{t^{*}}}$ is known from the filtration $\mathcal{F}_{t}$, and $R^{*}=\frac{S_{t^{*}+1}}{S_{t}}$ is log-normally distributed with parameters $\left(t^{*}+1-t\right)\left(r-\frac{\sigma^{2}}{2}\right)$ and $\left(t^{*}+1-t\right) \sigma^{2}$.

Let $f_{R^{*}}(x)=\frac{1}{x \sqrt{2 \pi} \sigma \sqrt{t^{*}+1-t}} \exp \left\{-\frac{\left[\ln x-\left(t^{*}+1-t\right)\left(r-\frac{\sigma^{2}}{2}\right)\right]^{2}}{2\left(t^{*}+1-t\right) \sigma^{2}}\right\}$ and $F_{R^{*}}(x)=\Phi\left(\frac{\ln x-\left(t^{*}+1-t\right)\left(r-\frac{\sigma^{2}}{2}\right)}{\sigma \sqrt{t^{*}+1-t}}\right)$ be the probability density and cumulative distribution functions of the random variable $R^{*}$, respectively.

Depending on $\frac{S_{t}}{S_{t^{*}}}$, the current year's actual credited return $\mathcal{R}_{t^{*}+1}$ can take one the following three possible values:

$$
\mathcal{R}_{t^{*}+1}=\left\{\begin{array}{lrr}
1+f & \text { when } & R^{*}<Q_{1} \\
1-\alpha+\alpha \frac{S_{t}}{S_{t^{*}}} R^{*} & \text { when } & Q_{1}<R^{*} \leq Q_{2} \\
1+c & \text { when } & R^{*}>Q_{2}
\end{array}\right.
$$


for $\frac{S_{t}}{S_{t^{*}}}>0$, where $Q_{1}=\frac{S_{t^{*}}}{S_{t}}\left(1+\frac{f}{\alpha}\right)$ and $Q_{2}=\frac{S_{t^{*}}}{S_{t}}\left(1+\frac{c}{\alpha}\right)$.

Taking the risk neutral expectation of $\mathcal{R}_{t^{*}+1}$ we get:

$$
\begin{aligned}
\mathbb{E}^{\mathbb{Q}}\left[\mathcal{R}_{t^{*}+1} \mid \mathcal{F}_{t}\right]= & \int_{0}^{Q_{1}}(1+f) f_{R^{*}}(x) d x+\int_{Q_{1}}^{Q_{2}}\left(1-\alpha+\alpha \frac{S_{t}}{S_{t^{*}}} x\right) f_{R^{*}}(x) d x \\
& +\int_{Q_{2}}^{\infty}(1+c) f_{R^{*}}(x) d x .
\end{aligned}
$$

It is clear that the integration in (4.2.5) is very similar to the one in (4.1.7), since we have the same log-normal distribution but with different parameters. Therefore we will omit some of the details. Similarly to (4.1.8), the first integral on the right-hand side of (4.2.5) is

$$
\begin{aligned}
\int_{0}^{Q_{1}}(1+f) f_{R^{*}}(x) d x & =(1+f) F_{R^{*}}\left(Q_{1}\right) \\
& =(1+f) \Phi\left(-h_{2}\right)
\end{aligned}
$$

Also, similarly to the steps of integration done in (4.1.9), the second term on the righthand side of (4.2.5) resolves to:

$$
\begin{aligned}
& \int_{Q_{1}}^{Q_{2}}\left(1-\alpha+\alpha \frac{S_{t}}{S_{t^{*}}} x\right) f_{R^{*}}(x) d x \\
& =(1-\alpha)\left[\Phi\left(\frac{\ln Q_{2}-\left(t^{*}+1-t\right)\left(r-\frac{\sigma^{2}}{2}\right)}{\sigma \sqrt{t^{*}+1-t}}\right)-\Phi\left(\frac{\ln Q_{1}-\left(t^{*}+1-t\right)\left(r-\frac{\sigma^{2}}{2}\right)}{\sigma \sqrt{t^{*}+1-t}}\right)\right] \\
& +\alpha \frac{S_{t}}{S_{t^{*}}} \int_{Q_{1}}^{Q_{2}} \frac{1}{\sqrt{2 \pi} \sigma \sqrt{t^{*}+1-t}} \exp \left\{-\frac{\left[\ln x-\left(t^{*}+1-t\right)\left(r-\frac{\sigma^{2}}{2}\right)\right]^{2}}{2\left(t^{*}+1-t\right) \sigma^{2}}\right\} d x .
\end{aligned}
$$

By applying the identity $\Phi(A)-\Phi(B)=\Phi(-B)-\Phi(-A)$, and the change of variable 
$y=\ln x$ with $d x=e^{y} d y$ on the second term we get:

$$
\begin{aligned}
& (1-\alpha)\left[\Phi\left(h_{2}\right)-\Phi\left(h_{4}\right)\right] \\
& +\alpha \frac{S_{t}}{S_{t^{*}}} \int_{\ln Q_{1}}^{\ln Q_{2}} \frac{1}{\sqrt{2 \pi} \sigma \sqrt{t^{*}+1-t}} \exp \left\{-\frac{\left[y-\left(t^{*}+1-t\right)\left(r+\frac{\sigma^{2}}{2}\right)\right]^{2}}{2\left(t^{*}+1-t\right) \sigma^{2}}+\left(t^{*}+1-t\right) r\right\} d y \\
& =(1-\alpha)\left[\Phi\left(h_{2}\right)-\Phi\left(h_{4}\right)\right]+\alpha \frac{S_{t}}{S_{t^{*}}} e^{\left(t^{*}+1-t\right) r}\left[\Phi\left(h_{1}\right)-\Phi\left(h_{3}\right)\right]
\end{aligned}
$$

and the third integral on the right-hand side of (4.2.5) is

$$
\begin{aligned}
\int_{Q_{2}}^{\infty}(1+c) f_{R^{*}}(x) d x & =(1+c)\left[1-F_{R^{*}}\left(Q_{2}\right)\right] \\
& =(1+c) \Phi\left(h_{4}\right)
\end{aligned}
$$

where $h_{1}, h_{2}, h_{3}$ and $h_{4}$ are given in Proposition 4.2 .

Consequently, by combining the results of the integrals from (4.2.6), (4.2.7) and (4.2.8), (4.2.5) becomes:

$$
\begin{aligned}
\mathbb{E}^{\mathbb{Q}}\left[\mathcal{R}_{t^{*}+1} \mid \mathcal{F}_{t}\right]=(1+f) \Phi\left(-h_{2}\right)+(1-\alpha)\left[\Phi\left(h_{2}\right)-\Phi\left(h_{4}\right)\right] \\
+\alpha \frac{S_{t}}{S_{t^{*}}} e^{\left(t^{*}+1-t\right) r}\left[\Phi\left(h_{1}\right)-\Phi\left(h_{3}\right)\right]+(1+c) \Phi\left(h_{4}\right) .
\end{aligned}
$$

Finally, we prove Proposition 4.2 by replacing the results of (4.2.9), (4.1.6) and (4.1.11) with their corresponding terms in (4.2.3).

Notice that the formula for the time- $t$ price $V_{t}^{C R}$ is true for all $0 \leq t^{*} \leq t<t^{*}+1 \leq T$. Especially in the case where we stand at any anniversary date of the contract, that is when $t$ is an integer such that $t=t^{*}$, we get $h_{s}=l_{s}$ for all $s=1,2,3,4$. It follows that $\mathbb{E}^{\mathbb{Q}}\left[\mathcal{R}_{t^{*}+1} \mid \mathcal{F}_{t}\right]$ in $(4.2 .9)$ becomes equal to $\mathbb{E}^{\mathbb{Q}}\left[\mathcal{R}_{t}\right]$ in $(4.1 .11)$, therefore we can directly 
conclude the following:

Corollary 4.1. Under the Black-Scholes framework, the price of an Annual Compound Ratchet EIA at any integer time $t=t^{*}$ has the following expression:

$$
\begin{gathered}
V_{t^{*}}^{C R}=e^{-\left(T-t^{*}\right) r} \prod_{i=1}^{t^{*}} \mathcal{R}_{i} \times\left[(1+f) \Phi\left(-l_{2}\right)+(1-\alpha)\left[\Phi\left(l_{2}\right)-\Phi\left(l_{4}\right)\right]+\right. \\
\left.\alpha e^{r}\left[\Phi\left(l_{1}\right)-\Phi\left(l_{3}\right)\right]+(1+c) \Phi\left(l_{4}\right)\right]^{T-t^{*}}
\end{gathered}
$$

where $\mathcal{R}_{i}$ is given by (4.1.5), and $l_{1}, l_{2}, l_{3}, l_{4}$ are given by Proposition 4.1 . 


\section{Chapter 5}

\section{Hedging Equity-Indexed Annuities}

This chapter presents how to apply the hedging strategies derived from the Greeks to the Annual Compound Ratchet EIA contract. Firstly, the Delta-hedging strategy is implemented. Then due to discretization, the hedge is not perfect since basis risk still has to be dealt with. Therefore, hedging errors arise and the Gamma-hedging strategy is incorporated to improve the Delta-hedging strategy by reducing the magnitude of these errors. Yet, in reality basis risk always exists and hedging errors represent a supplemental cost of the hedging portfolio for the issuer. With the purpose of protecting the issuer from this additional cost, and by trying to reproduce the pattern of the changing rates based on what is observed in practice, a new approach is proposed and implemented to eliminate the risk born by the issuer from applying the hedging strategies.

\subsection{Hedging of Compound Annual Ratchet EIA}

A Compound Ratchet EIA's payoff is convex with respect to the stock price $S$ and thus it is considered a non-linear derivative security in its underlying. Therefore it needs to be hedged using a dynamic hedging strategy, such as the one presented in detail in Chapter 3 . 


\subsubsection{Delta-hedging strategy}

First, the Delta-hedging strategy previously derived is implemented from the perspective of issuers of annual CR EIA contracts with $T$ years until maturity. The replicating portfolio is set such that it reproduces the payoff of the EIA contract at any time $t$; $0 \leq t \leq T$. The issuer of this contract hedges his initial position by building a replicating portfolio, which is also referred to as a hedging portfolio. Recall that the objective of this strategy is to eliminate the risk inherent to the small variations in the underlying stock price, and thus neutralizing the sensitivity of the overall position in the EIA contract and the replicating portfolio to changes in the underlying.

Under the Delta-hedging strategy, as explained in Section 3.2.1, the replicating portfolio is composed of only stocks and money market accounts; $\psi_{t}^{\Delta}=\left\{\psi_{S, t}^{\Delta}, \psi_{M, t}^{\Delta}\right\}$, such that the portion of shares bought $\psi_{S, t}^{\Delta}$ is determined by the Delta of CR EIA contract, with the remaining being the portion $\psi_{M, t}^{\Delta}$ invested in the money market account. Thus the value of the Delta-hedging portfolio is given by:

$$
\begin{aligned}
V_{t}^{\Delta} & =\psi_{S, t}^{\Delta} S_{t}+\psi_{M, t}^{\Delta} M_{t} \\
& =\Delta_{t}^{C R} S_{t}+\left(V_{t}^{C R}-\Delta_{t}^{C R} S_{t}\right)
\end{aligned}
$$

for all $t$, where $\Delta_{t}^{C R}$ is the Delta of the CR EIA to be determined next, and $V_{t}^{C R}$ is the value of the CR EIA given by (4.2.1).

Proposition 5.1. Under the Black-Scholes framework, where the time- $t$ value of an $A n$ nual Compound Ratchet EIA contract is given by Proposition 4.2, a closed-form expression 
of the Delta of the CR EIA contract is given as follows:

$$
\begin{aligned}
\Delta_{t}^{C R}= & e^{-(T-t) r} \prod_{i=1}^{t^{*}} \mathcal{R}_{i} \\
\times & {\left[(1+f) \frac{-1}{S_{t} \sigma \sqrt{t^{*}+1-t}} \phi\left(-h_{2}\right)+(1-\alpha) \frac{1}{S_{t} \sigma \sqrt{t^{*}+1-t}}\left[\phi\left(h_{2}\right)-\phi\left(h_{4}\right)\right]\right.} \\
& +\frac{\alpha}{S_{t^{*}}} e^{\left(t^{*}+1-t\right) r}\left[\Phi\left(h_{1}\right)-\Phi\left(h_{3}\right)\right]+\frac{\alpha}{S_{t^{*}}} e^{\left(t^{*}+1-t\right) r} \frac{1}{\sigma \sqrt{t^{*}+1-t}}\left[\phi\left(h_{1}\right)-\phi\left(h_{3}\right)\right] \\
& \left.+(1+c) \frac{1}{S_{t} \sigma \sqrt{t^{*}+1-t}} \phi\left(h_{4}\right)\right] \\
& \times\left(\mathbb{E}^{\mathbb{Q}}\left[\mathcal{R}_{i}\right]\right)^{T-t^{*}-1},
\end{aligned}
$$

for all $0<t \leq T$, and

$$
\Delta_{0}^{C R}=0,
$$

where $h_{1}, h_{2}, h_{3}$ and $h_{4}$ are given in Proposition 4.2. $\mathcal{R}_{i}$ and $\mathbb{E}^{\mathbb{Q}}\left[\mathcal{R}_{i}\right]$ are given by (4.1.5) and (4.1.11), respectively. Also, $\phi()$ is the standard normal density function.

Proof. From (3.1) and (4.2.1) we have:

$$
\begin{aligned}
\Delta_{t}^{C R} & =\frac{\partial}{\partial S_{t}} V_{t}^{C R} \\
& =e^{-(T-t) r} \prod_{i=1}^{t^{*}} \mathcal{R}_{i} \times\left(\mathbb{E}^{\mathbb{Q}}\left[\mathcal{R}_{i}\right]\right)^{T-t^{*}-1} \times \frac{\partial}{\partial S_{t}} \mathbb{E}^{\mathbb{Q}}\left[\mathcal{R}_{t^{*}+1} \mid \mathcal{F}_{t}\right]
\end{aligned}
$$

where, by (4.2.9) we have:

$$
\begin{aligned}
& \frac{\partial}{\partial S_{t}} \mathbb{E}^{\mathbb{Q}}\left[\mathcal{R}_{t^{*}+1} \mid \mathcal{F}_{t}\right]=\frac{\partial}{\partial S_{t}} {\left[(1+f) \Phi\left(-h_{2}\right)+(1-\alpha)\left[\Phi\left(h_{2}\right)-\Phi\left(h_{4}\right)\right]\right.} \\
&\left.+\alpha \frac{S_{t}}{S_{t^{*}}} e^{\left(t^{*}+1-t\right) r}\left[\Phi\left(h_{1}\right)-\Phi\left(h_{3}\right)\right]+(1+c) \Phi\left(h_{4}\right)\right] .
\end{aligned}
$$

Now since $h_{1}=\frac{-\ln \left(\frac{S_{t^{*}}}{S_{t}}\left(1+\frac{f}{\alpha}\right)\right)+\left(t^{*}+1-t\right)\left(r+\frac{\sigma^{2}}{2}\right)}{\sigma \sqrt{t^{*}+1-t}}$ with $h_{2}=h_{1}-\sigma \sqrt{t^{*}+1-t}$ we have that $\frac{\partial}{\partial S_{t}}\left(h_{1}\right)=\frac{\partial}{\partial S_{t}}\left(h_{2}\right)=\frac{1}{S_{t} \sigma \sqrt{t^{*}+1-t}}$. 
Similarly for $h_{3}$ and $h_{4}$, in general we have

$$
\frac{\partial}{\partial S_{t}}\left(h_{s}\right)=\frac{1}{S_{t} \sigma \sqrt{t^{*}+1-t}}
$$

for any $s=1,2,3,4$. It follows that by the Chain rule we have:

$$
\frac{\partial}{\partial S_{t}}\left(\Phi\left(h_{s}\right)\right)=\frac{1}{S_{t} \sigma \sqrt{t^{*}+1-t}} \phi\left(h_{s}\right)
$$

Thus (5.1.5) becomes:

$$
\begin{aligned}
& (1+f) \frac{-1}{S_{t} \sigma \sqrt{t^{*}+1-t}} \phi\left(-h_{2}\right)+(1-\alpha)\left[\frac{1}{S_{t} \sigma \sqrt{t^{*}+1-t}} \phi\left(h_{2}\right)-\frac{1}{S_{t} \sigma \sqrt{t^{*}+1-t}} \phi\left(h_{4}\right)\right] \\
& +\alpha \frac{1}{S_{t^{*}}} e^{\left(t^{*}+1-t\right) r}\left[\Phi\left(h_{1}\right)-\Phi\left(h_{3}\right)\right]+\alpha \frac{S_{t}}{S_{t^{*}}} e^{\left(t^{*}+1-t\right) r}\left[\frac{1}{S_{t} \sigma \sqrt{t^{*}+1-t}} \phi\left(h_{1}\right)-\frac{1}{S_{t} \sigma \sqrt{t^{*}+1-t}} \phi\left(h_{3}\right)\right] \\
& +(1+c) \frac{1}{S_{t} \sigma \sqrt{t^{*}+1-t}} \phi\left(h_{4}\right) \\
& =(1+f) \frac{-1}{S_{t} \sigma \sqrt{t^{*}+1-t}} \phi\left(-h_{2}\right)+(1-\alpha) \frac{1}{S_{t} \sigma \sqrt{t^{*}+1-t}}\left[\phi\left(h_{2}\right)-\phi\left(h_{4}\right)\right] \\
& +\frac{\alpha}{S_{t^{*}}} e^{\left(t^{*}+1-t\right) r}\left[\Phi\left(h_{1}\right)-\Phi\left(h_{3}\right)\right]+\frac{\alpha}{S_{t^{*}}} e^{\left(t^{*}+1-t\right) r} \frac{1}{\sigma \sqrt{t^{*}+1-t}}\left[\phi\left(h_{1}\right)-\phi\left(h_{3}\right)\right] \\
& +(1+c) \frac{1}{S_{t} \sigma \sqrt{t^{*}+1-t}} \phi\left(h_{4}\right) .
\end{aligned}
$$

Therefore, by replacing (5.1.7) into (5.1.4) we prove (5.1.2).

Additionally, from Definition 3.1 we know that $\Delta_{0}^{C R}=\frac{\partial}{\partial S_{t}} V_{0}^{C R}$, where the expression of $V_{0}^{C R}$ in (4.1.3) does not depend on $S_{t}$, therefore we have that $\frac{\partial}{\partial S_{t}} V_{0}^{C R}=0$. By that we have proved (5.1.3). 


\subsubsection{Delta-hedging Errors}

The Delta-hedging strategy applied continuously leads to a perfect hedge. This can be clearly concluded from (5.1.1), since the value of the Delta-hedging portfolio always equals that of the CR EIA contract, $V_{t}^{\Delta}=V_{t}^{C R}$ for all $0 \leq t \leq T$, especially at maturity to cover the outstanding liability where $V_{T}^{\Delta}=\Lambda^{C R}$, with $\Lambda^{C R}$ being the payoff of the CR EIA given in (4.1.1).

However, as explained thoroughly in Section 3.3, the hedge can only be partial in reality, and the discretization of the continuous-time model leads to Delta-hedging errors. Assuming that we have $m$ number of trading dates per year, we can divide our time horizon into $m T$ equally distant periods such that $t=0, \frac{1}{m}, \frac{2}{m}, \ldots, T+\frac{m-1}{m}, T$.

At $t=0$, the writer of the CR EIA sells the contract for its time-zero price $V_{0}^{C R}$. No investment in the stocks is done initially since $\Delta_{0}^{C R}=0$, therefore all the proceedings are invested in the money market account earning the risk free rate $r$, and we have $V_{0}^{\Delta}=V_{0}^{C R}$. Then, at each trading trade $t \in\left\{\frac{1}{m}, \frac{2}{m}, \ldots, T+\frac{m-1}{m}, T\right\}$, the writer rebalances his replicating portfolio such that the proportion invested in $S_{t}$ is set to the Delta of the CR EIA, $\Delta_{t}^{C R}$. It follows that the value of the Delta-hedging portfolio right before each periodic re-balancing is:

$$
V_{t^{-}}^{\Delta}=\Delta_{t-\frac{1}{m}}^{C R} S_{t}+\left(V_{t-\frac{1}{m}}^{C R}-\Delta_{t-\frac{1}{m}}^{C R} S_{t-\frac{1}{m}}\right) e^{\frac{r}{m}}
$$

and its value after each periodic re-balancing becomes:

$$
\begin{aligned}
V_{t}^{\Delta} & =\Delta_{t}^{C R} S_{t}+\left(V_{t}^{C R}-\Delta_{t}^{C R} S_{t}\right) \\
& =V_{t}^{C R} .
\end{aligned}
$$


As a result, the periodic Delta-hedging errors can be obtained from (3.3.1) with $V_{t^{-}}^{\psi}=$ $V_{t^{-}}^{\Delta}$ and $V_{t}=V_{t}^{C R}$ to get:

$$
\begin{aligned}
P H E_{t}^{\Delta} & =V_{t^{-}}^{\Delta}-V_{t}^{C R} \\
& =\Delta_{t-\frac{1}{m}}^{C R} S_{t}+\left(V_{t-\frac{1}{m}}^{C R}-\Delta_{t-\frac{1}{m}}^{C R} S_{t-\frac{1}{m}}\right) e^{\frac{r}{m}}-V_{t}^{C R} .
\end{aligned}
$$

Note that the discrepancy that leads to these periodic Delta-hedging errors only arises from the discretization process, since if we let $m$ go to infinity in (5.1.8), that is making the discrete time periods infinitesimally small, we get back the continuous-time model and the Delta-hedging errors go to zero as expected.

In fact, the mean of the present value at time-zero of each periodic Delta-hedging error is zero under the risk neutral measure, since

$$
\begin{aligned}
\mathbb{E}^{\mathbb{Q}}\left[e^{-r t} P H E_{t}^{\Delta} \mid \mathcal{F}_{0}\right]= & \Delta_{t-\frac{1}{m}}^{C R} \mathbb{E}^{\mathbb{Q}}\left[e^{-r t} S_{t} \mid \mathcal{F}_{0}\right]+\mathbb{E}^{\mathbb{Q}}\left[e^{-r\left(t-\frac{1}{m}\right)} V_{t-\frac{1}{m}}^{C R} \mid \mathcal{F}_{0}\right] \\
& \quad-\Delta_{t-\frac{1}{m}}^{C R} \mathbb{E}^{\mathbb{Q}}\left[e^{-r\left(t-\frac{1}{m}\right)} S_{t-\frac{1}{m}} \mid \mathcal{F}_{0}\right]-\mathbb{E}^{\mathbb{Q}}\left[e^{-r t} V_{t}^{C R} \mid \mathcal{F}_{0}\right] \\
= & \Delta_{t-\frac{1}{m}}^{C R} S_{0}+V_{0}^{C R}-\Delta_{t-\frac{1}{m}}^{C R} S_{0}-V_{0}^{C R}=0 .
\end{aligned}
$$

It also follows that the present value of the Total Delta-hedging Errors, denoted by $T H E_{0}^{\Delta}$ is a martingale under the Black-Scholes model with a mean of zero. Therefore, to assess the performance of the Delta-hedging strategy we analyze the distribution of the present value at time-zero of the sum of all the periodic Delta-hedging errors, which from (3.3.2) has the following expression:

$$
\begin{aligned}
T H E_{0}^{\Delta} & =\sum_{t=0}^{T-1} \sum_{i=1}^{m} e^{-\left(t+\frac{i}{m}\right) r} P H E_{t+\frac{i}{m}}^{\Delta} \\
& =\sum_{t=0}^{T-1} \sum_{i=1}^{m} e^{-\left(t+\frac{i}{m}\right) r}\left[\Delta_{t+\frac{i-1}{m}}^{C R} S_{t+\frac{i}{m}}+\left(V_{t+\frac{i-1}{m}}^{C R}-\Delta_{t+\frac{i-1}{m}}^{C R} S_{t+\frac{i-1}{m}}\right) e^{\frac{r}{m}}-V_{t+\frac{i}{m}}^{C R}\right] .
\end{aligned}
$$


Our numerical analyses considers by default a 7-year Annual Compound Ratchet EIA contract $(T=7)$ bounded by a local floor rate of $0 \%(f=0)$ and a local cap rate of $100 \%$ $(c=1)$, where the dynamics of the returns on the index are governed by a Log-Normal distribution with parameters $\mu=8 \%$ and $\sigma=20 \%$. We assume a $4 \%$ risk free rate of return $(r=4 \%)$ and weekly portfolio re-balancing $(m=52)$. Additionally, we choose the participation rate $\alpha$ such that the time-zero price of the contract $V_{0}^{C R}$ is 1 monetary unit, to get $\alpha=39.5 \%$. Under these assumptions, we simulate 25, 000 sample paths of the stock index returns under the discretization model presented in Section 3.3.3, and apply the Delta-hedging strategy by performing Monte Carlo simulations.

\section{Delta Hedging}

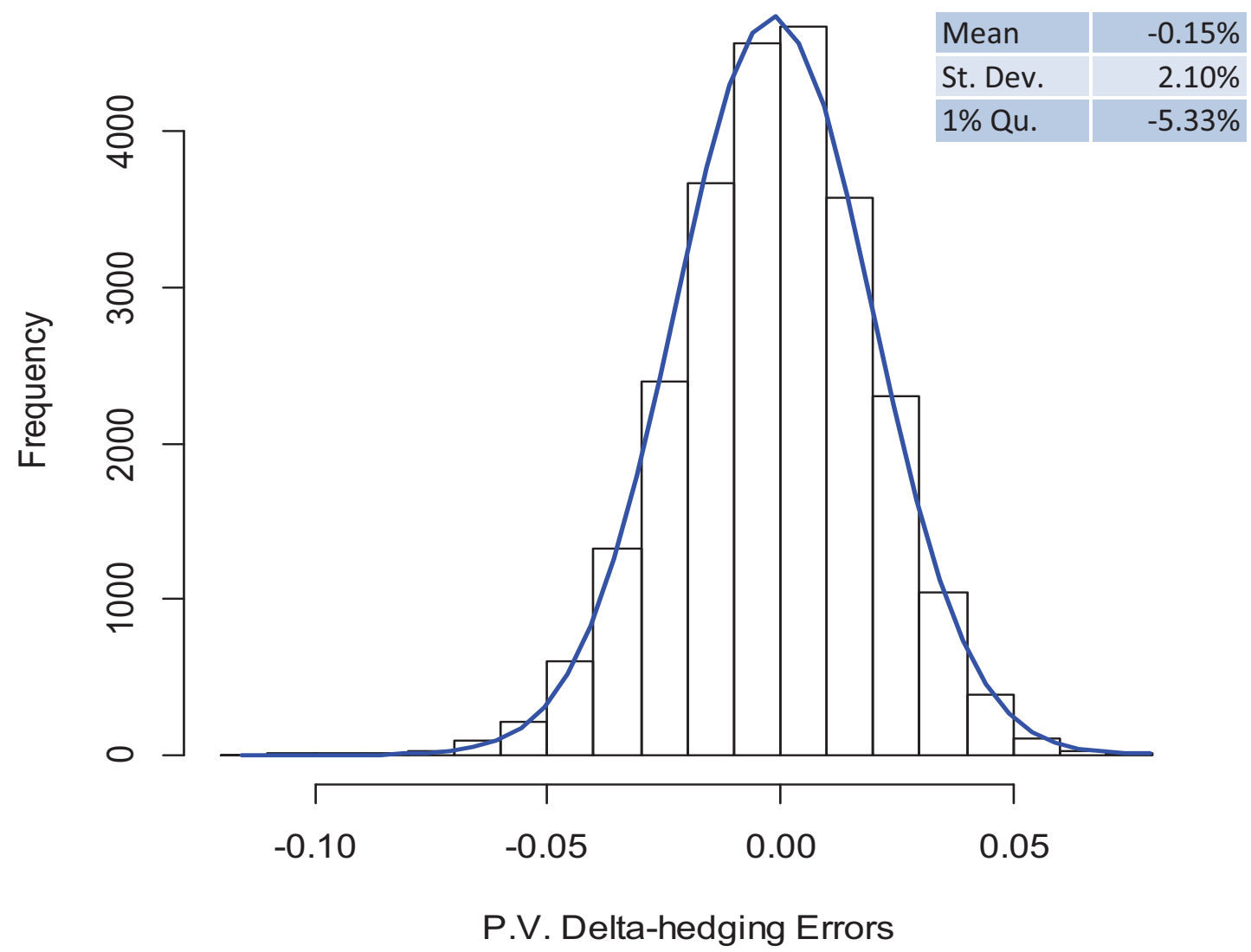

Figure 5.1: Present value of Delta-hedging errors. 
The empirical distribution of the present value of Delta-hedging errors is illustrated in Figure 5.1. Recall that these hedging errors represent an added cost to build up the replicating portfolio. The empirical distribution of $T H E_{0}^{\Delta}$ is centered around the mean $(-0.15 \%)$ which is very close to zero. This shows that the average cost of the hedging portfolio is almost zero, and thus on average the hedging strategy is a fair game. Additionally, the empirical distribution has a relatively low standard deviation (2.1\%), which means that the cost of the hedging strategy is somehow predictable and thus does not vary too much. Moreover, by (5.1.8), negative hedging errors represent a loss for the issuer resulting from the application of the hedging strategy. Naturally, it is expected that issuers seek to minimize this loss, hence the heaviness of the left tail is to be examined. Table 5.1 lists different quantile values of the distribution of $T H E_{0}^{\Delta}$. These values can be used to set a maximum tolerable loss for the issuers depending on their risk appetite. For example, the Delta-hedging strategy will cost the issuer more than $5.33 \%$ of the EIA with $1 \%$ probability.

\begin{tabular}{c||c|c|c|c|c|c|c|c} 
Quantiles & $1 \%$ & $2 \%$ & $5 \%$ & $25 \%$ & $50 \%$ & $75 \%$ & $95 \%$ & $99 \%$ \\
\hline \hline THE $_{0}^{\Delta}$ (in \%) & -5.33 & -4.65 & -3.69 & -1.5 & -0.08 & 1.28 & 3.19 & 5.06
\end{tabular}

Table 5.1: Quantiles of the distribution of $T H E_{0}^{\Delta}$, in percentages.

To summarize, it can be said that the Delta-hedging strategy works well under the Black-Scholes framework. However, one can resort to the Gamma-hedging strategy as a way to reduce the effect of discretization and thus improve the performance of the hedge.

\subsubsection{Gamma-hedging Strategy}

As explained in Section 3.2.2, the Gamma-hedging strategy incorporates a third asset to the hedging portfolio, a European Call option, which will help reduce the exposure to risk due to wide variations in the stock price resulting from the discretization process. 
It is first assumed that European Call options has maturity $\frac{1}{m}$ (in years) and strike price $S_{t}$, and is always available on the financial market at any time $t \in\left\{\frac{1}{m}, \frac{2}{m}, \ldots, T+\frac{m-1}{m}, T\right\}$, and the replicating portfolio $\psi_{t}^{\Gamma}$ has the following composition $\psi_{t}^{\Gamma}=\left\{\psi_{S, t}^{\Gamma}, \psi_{C, t}^{\Gamma}, \psi_{M, t}^{\Gamma}\right\}$. Then to construct this replicating portfolio under the Gamma-hedging strategy, both the Delta and Gamma of the portfolio are matched to those of the CR EIA respectively, as seen in (3.2.8), dynamically at the beginning of each period such that:

$$
\begin{aligned}
& \Gamma_{t}^{C R}=\psi_{C, t}^{\Gamma} \Gamma_{t}^{C}, \\
& \Delta_{t}^{C R}=\psi_{S, t}^{\Gamma}+\psi_{C, t}^{\Gamma} \Delta_{t}^{C} .
\end{aligned}
$$

It follows that by rearranging (5.1.10) one can solve for the portions invested in stocks, options, and money market account to get:

$$
\begin{aligned}
\psi_{C, t}^{\Gamma} & =\frac{\Gamma_{t}^{C R}}{\Gamma_{t}^{C}} \\
\psi_{S, t}^{\Gamma} & =\Delta_{t}^{C R}-\left(\frac{\Gamma_{t}^{C R}}{\Gamma_{t}^{C}}\right) \Delta_{t}^{C}, \\
\psi_{M, t}^{\Gamma} & =\left(V_{t}^{C R}-\psi_{S, t}^{\Gamma} S_{t}-\psi_{C, t}^{\Gamma} V_{t}^{C}\right) \frac{1}{M_{t}},
\end{aligned}
$$

where $V_{t}^{C}, \Delta_{t}^{C}$ and $\Gamma_{t}^{C}$ are the time- $t$ value, Delta and Gamma of the European Call option given in (2.5.2), (3.2.5) and (3.2.9), respectively. $V_{t}^{C R}$ is the time- $t$ value of the CR EIA contract given in Proposition $4.2, \Delta_{t}^{C R}$ is the Delta of the CR EIA given in Proposition 5.1, and $\Gamma_{t}^{C R}$ is the Gamma of the CR EIA given in the following proposition.

Proposition 5.2. Under the Black-Scholes framework the Gamma of a Compound Ratchet EIA contract has the following expression: 


$$
\begin{aligned}
& \Gamma_{t}^{C R}=e^{-(T-t) r} \prod_{i=1}^{t^{*}} \mathcal{R}_{i} \\
& \times {\left[(1+f) \frac{1}{S_{t}^{2} \sqrt{t^{*}+1-t} \sigma} \phi\left(-h_{2}\right)\left(1+\frac{h_{2}}{\sqrt{t^{*}+1-t} \sigma}\right)\right.} \\
&+(1-\alpha) \frac{1}{S_{t}^{2} \sqrt{t^{*}+1-t} \sigma}\left[\phi\left(h_{4}\right)\left(1+\frac{h_{4}}{\sqrt{t^{*}+1-t} \sigma}\right)-\phi\left(h_{2}\right)\left(1+\frac{h_{2}}{\sqrt{t^{*}+1-t} \sigma}\right)\right] \\
&+e^{\left(t^{*}+1-t\right) r} \frac{\alpha}{S_{t^{*}} S_{t} \sqrt{t^{*}+1-t} \sigma}\left[\phi\left(h_{1}\right)-\phi\left(h_{3}\right)\right] \\
&+e^{\left(t^{*}+1-t\right) r} \frac{\alpha}{S_{t^{*}} S_{t}\left(t^{*}+1-t\right) \sigma^{2}}\left[h_{3} \phi\left(h_{3}\right)-h_{1} \phi\left(h_{1}\right)\right] \\
&\left.+(1+c) \frac{-1}{S_{t}^{2} \sqrt{t^{*}+1-t} \sigma} \phi\left(h_{4}\right)\left(1+\frac{h_{4}}{\sqrt{t^{*}+1-t} \sigma}\right)\right] \\
& \times\left(\mathbb{E}^{\mathbb{Q}}\left[\mathcal{R}_{i}\right]\right)^{T-t^{*}-1},
\end{aligned}
$$

for all $0<t \leq T$, and

$$
\Gamma_{0}^{C R}=0
$$

where $h_{1}, h_{2}, h_{3}$ and $h_{4}$ are given in Proposition 4.2. $\mathcal{R}_{i}$ and $\mathbb{E}^{\mathbb{Q}}\left[\mathcal{R}_{i}\right]$ are given by (4.1.5) and (4.1.11), respectively.

Proof. From Definition 3.2.7 and Proposition 5.1 we have:

$$
\begin{aligned}
\Gamma_{t}^{C R} & =\frac{\partial}{\partial S_{t}} \Delta_{t}^{C R} \\
& =e^{-(T-t) r} \prod_{i=1}^{t^{*}} \mathcal{R}_{i} \times\left(\mathbb{E}^{\mathbb{Q}}\left[\mathcal{R}_{i}\right]\right)^{T-t^{*}-1} \times \frac{\partial^{2}}{\partial\left(S_{t}\right)^{2}} \mathbb{E}^{\mathbb{Q}}\left[\mathcal{R}_{t^{*}+1} \mid \mathcal{F}_{t}\right]
\end{aligned}
$$

Now by the chain rule, we have in general for any $s=1,2,3,4$ :

$$
\frac{\partial}{\partial S_{t}}\left(\phi\left(h_{s}\right)\right)=\frac{1}{S_{t} \sigma \sqrt{t^{*}+1-t}}\left(-h_{s}\right) \phi\left(h_{s}\right)
$$


Then by using (4.2.9), (5.1.15) and (5.1.6) to derive (5.1.14), we prove (5.1.12). The details of integration are omitted because they are somewhat similar to those done in the proof of Proposition 5.1.

Additionally, from Definition 3.2.7 and (5.1.3) we have $\Gamma_{0}^{C R}=\frac{\partial}{\partial S_{t}} \Delta_{0}^{C R}=0$, and by that we have proved (5.1.13).

\subsubsection{Gamma-hedging Errors}

The Gamma-hedging strategy also produces a partial hedge due to the discretization, which leads to Gamma-hedging errors. Following the same discrete time setting as in Section 5.1.2, this strategy can be summarized as follows.

At $t=0$, the issuer of the annual CR EIA sells the contract for its time-zero price $V_{0}^{C R}$. No investment in the stocks nor options is made initially since, from (5.1.3) and (5.1.13) we have $\Delta_{0}^{C R}=0$ and $\Gamma_{0}^{C R}=0$. Therefore, all the proceedings are invested in the money market account earning the risk-free rate, such that

$$
V_{0}^{\Gamma}=V_{0}^{C R}
$$

Then, at each trading date $t \in\left\{\frac{1}{m}, \frac{2}{m}, \ldots, T+\frac{m-1}{m}, T\right\}$, the writer re-balances his hedge by re-adjusting the proportions of the replicating portfolio such that both the Delta and Gamma of this portfolio remain equal to those of the CR EIA. It follows that the value of the Gamma-hedging portfolio right before each periodic re-balancing is:

$$
\begin{aligned}
V_{t^{-}}^{\Gamma}= & {\left[\Delta_{t-\frac{1}{m}}^{C R}-\left(\frac{\Gamma_{t-\frac{1}{m}}^{C R}}{\Gamma_{t-\frac{1}{m}}^{C}}\right) \Delta_{t-\frac{1}{m}}^{C}\right] \times S_{t}+\left[\frac{\Gamma_{t-\frac{1}{m}}^{C R}}{\Gamma_{t-\frac{1}{m}}^{C}}\right] \times V_{t}^{C} } \\
& +\left[V_{t-\frac{1}{m}}^{C R}-\left(\Delta_{t-\frac{1}{m}}^{C R}-\left(\frac{\Gamma_{t-\frac{1}{m}}^{C R}}{\Gamma_{t-\frac{1}{m}}^{C}}\right) \Delta_{t-\frac{1}{m}}^{C}\right) \times S_{t-\frac{1}{m}}-\left(\frac{\Gamma_{t-\frac{1}{m}}^{C R}}{\Gamma_{t-\frac{1}{m}}^{C}}\right) \times V_{t-\frac{1}{m}}^{C}\right] \times e^{\frac{r}{m}},
\end{aligned}
$$


and its value after each periodic re-balancing becomes:

$$
\begin{aligned}
V_{t}^{\Gamma}= & {\left[\Delta_{t}^{C R}-\left(\frac{\Gamma_{t}^{C R}}{\Gamma_{t}^{C}}\right) \Delta_{t}^{C}\right] \times S_{t}+\left[\frac{\Gamma_{t}^{C R}}{\Gamma_{t}^{C}}\right] \times V_{t}^{C} } \\
& \quad+V_{t}^{C R}-\left(\Delta_{t}^{C R}-\left(\frac{\Gamma_{t}^{C R}}{\Gamma_{t}^{C}}\right) \Delta_{t}^{C}\right) \times S_{t}-\left(\frac{\Gamma_{t}^{C R}}{\Gamma_{t}^{C}}\right) \times V_{t}^{C} \\
= & V_{t}^{C R} .
\end{aligned}
$$

It follows that the periodic Gamma-hedging errors can be obtained from (3.3.1) with $V_{t^{-}}^{\psi}=V_{t^{-}}^{\Gamma}$ and $V_{t}=V_{t}^{C R}$ to get:

$$
P H E_{t}^{\Gamma}=V_{t^{-}}^{\Gamma}-V_{t}^{C R}
$$

and from (5.1.16), we have $P H E_{0}^{\Gamma}=0$.

Similarly to (5.1.9), under the risk neutral measure $\mathbb{Q}$, the mean of the present value at time-zero of each periodic Gamma-hedging error is zero, that is $\mathbb{E}^{\mathbb{Q}}\left[e^{-r t} P H E_{t}^{\Gamma} \mid \mathcal{F}_{0}\right]=0$. It follows that the present value of the sum of all periodic Gamma-hedging errors is a martingale under the Black-Scholes model with a mean of zero, and has the following expression:

$$
\begin{aligned}
T H E_{0}^{\Gamma} & =\sum_{t=0}^{T-1} \sum_{i=1}^{m} e^{-\left(t+\frac{i}{m}\right) r} P H E_{t+\frac{i}{m}}^{\Gamma} \\
& =\sum_{t=0}^{T-1} \sum_{i=1}^{m} e^{-\left(t+\frac{i}{m}\right) r}\left(V_{\left(t+\frac{i}{m}\right)^{-}}^{\Gamma}-V_{t+\frac{i}{m}}^{C R}\right) .
\end{aligned}
$$

To assess the performance of the Gamma-hedging strategy, the empirical distribution of $\mathrm{THE}_{0}^{\Gamma}$ is to be analyzed. Therefore, 25,000 Monte Carlo simulations of the Gammahedging strategy are done on the 7-year Annual Compound Ratchet EIA with the default parameter set considered in Section 5.1.2, and the resulting Gamma-hedging errors of (5.1.18) are extracted. 


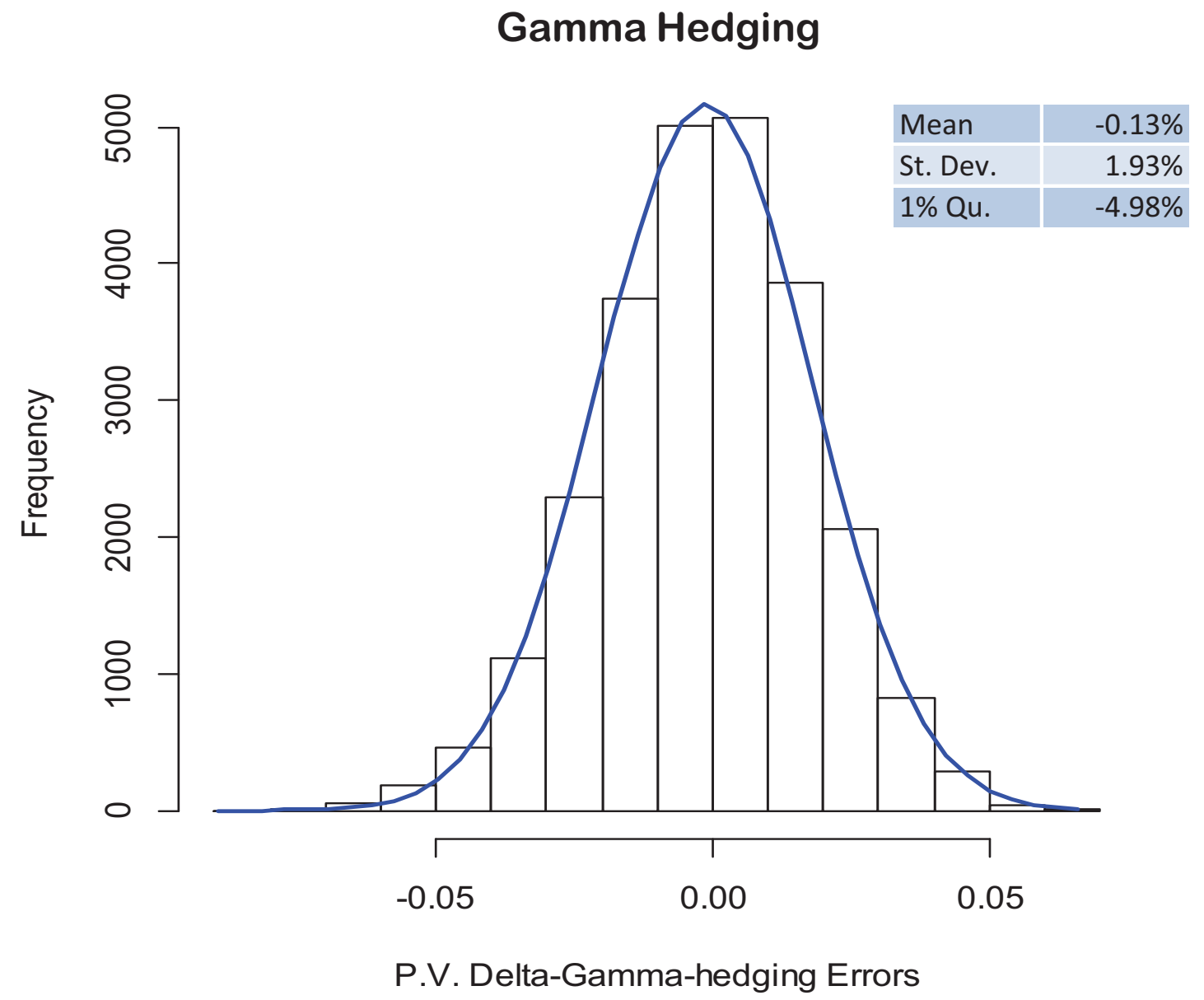

Figure 5.2: Present value of Gamma-hedging errors.

Figure 5.2 represents the empirical distribution of the present value of Gamma-hedging errors. In-the-money Call options with periodic maturities are used. The analysis of $T H E_{o}^{\Gamma}$ shows that this hedge is also on average is fair since the mean is very close to zero $(-0.13 \%)$. Additionally, we can notice an improvement over the Delta-hedging strategy given that the standard deviation is also lower to $1.93 \%$, leading to a somehow predictable cost. Moreover, another improvement can be noted as the cost incurred by the issuer to use this Gamma-hedging strategy will decrease by $0.352 \%$, to $4.98 \%$ per unit of invest- 
ment in the EIA, considering $1 \%$ worst loss.

As a result, the application of the Gamma-hedging strategy reduces the risk born by the issuer of the EIA, and as anticipated, improves the performance of the Delta-hedging strategy by reducing the magnitude of the errors, and thus lowering the cost of the hedge.

Both strategies hedge the risk against changes in the stock price, one can also hedge against changes in volatility using the Greek letter Vega, denoted by $\nu$. However, Hardy (2003) shows that the sensitivity of different EIA contract types to volatility is similar, except for the Annual Compound Ratchet case with the embedded floor and cap rates, where it is less sensitive since the volatility of the index is limited by the floor and cap rates. Moreover, the Black-Scholes model assumes a constant volatility, so it there will be no changes in volatility throughout the contract term. For this reason, we do not extend the hedging strategy to the Vega in this thesis.

\subsection{Dynamic Risk Management Strategy}

By improving the hedging strategies we only manage to reduce the cost, but not totally eliminate the risk. In this section, we shall propose a new approach that further reduces the risk of hedging and thus protects the issuer as much as possible from the hedging errors.

This new approach is inspired from what we actually see in practice. Observe for example the product "Allianz 222 Annuity "sold by Allianz Life Insurance Company of North America. Different allocation options are offered, including the Annual Point-topoint with either a spread or a cap, and different index options like the S\&P 500 Index, Nasdaq-100 Index, Russel-2000 Index, and many more. For this specific product, the participation rate is set to $100 \%$ and guaranteed for the life of the contract. In particular, for the option where a cap rate is applied, the insurance company is retaining the right 
of changing the cap rate on each contract anniversary, yet with a promise of not going below a predetermined minimum throughout the term of the contract as a guarantee for the buyer. Similarly for the option where a spread is deducted, the issuer changes the spread rate, say yearly, however a maximum yearly spread rate is specified at inception. Other products also include the option of changing the participation rate instead on each anniversary date.

As a result, a new strategy that aims at reducing the hedging errors by incorporating the changing rates' pattern is proposed. As previously mentioned, whenever the periodic hedging errors are negative, the issuer is obliged to inject money into the hedging portfolio. The series of inflows represent a loss incurred by the issuer since he must use external resources to maintain the hedge until the end of the contract. To cover up for his loss, the issuer could, for example, initially increase the cost of the EIA by the estimated present value of all periodic cash flows, or decrease the final payoff by the future value of the net periodic injections, which in both ways, will not be appealing to investors, and thus makes it hard to compete with other issuers. In all cases, issuers have to make sure that they retain their customers. An important reference on this matter is presented by Gaillardetz and Lakhmiri (2011). They propose a new premium principle for equity-linked products by loading the premium through finding a loaded participation rate based on the hedging errors. In a similar fashion, a subtle method is proposed here to transfer the loss from the issuer to the buyer throughout the whole term of the contract. In other words, instead of resorting to external resources for injecting money into the hedging portfolio whenever needed, we propose transferring the required amount from the policyholder to the issuer (whenever the periodic error is negative, i.e. cash inflow) and from the issuer to the policyholder (whenever the periodic error is positive, i.e. cash outflow) through the pricing parameter. This requires changing the value of the EIA contract dynamically, say at anniversary dates.

In general, the parameters that the writer of an EIA can control and that change the 
value of the contract are the participation rate $\alpha$, the local cap rate $c$, and the local spread rate $s$. Let $\underline{\theta_{t}}=\left\{\underline{\alpha_{t}}, \underline{c_{t}}, \underline{s_{t}}\right\}$ be the vector of parameters indicating the value of these rates at any time $t$. That is, $\underline{\alpha_{t}}=\left\{\alpha_{0}, \alpha_{1}, \ldots, \alpha_{t}\right\}$, and similarly for $\underline{c_{t}}$ and $\underline{s_{t}}$. The value of the EIA contract will be dependent on this vector $\underline{\theta}_{t}$. In fact, the most common EIA contracts sold on the market are offering one varying parameter, while the two others, whenever applicable, remain fixed.

More specifically, this dynamic risk management method coupled with any of the previously proposed hedging strategies will be applied as follows: Assume at $t=0$ the issuer sells the Annual Compound Ratchet EIA, and use the proceedings to hedge his long position in the contract. The chosen changing rate in $\underline{\theta_{0}}$ is initially determined such that

$$
V_{0}^{C R}\left(\underline{\theta_{0}}\right)=1
$$

Then at each trading date, whether the Delta or Gamma-hedging strategy is used, the proportions of the replicating portfolio are re-balanced accordingly and the amount of periodic error incurred is recorded. These periodic errors are accumulate and aggregated until the end of the year to get the future value of the total amount of error incurred during that year, denoted by $Y H E_{t}^{\psi}$ and given by

$$
Y H E_{t}^{\psi}=\sum_{i=1}^{m} e^{\left(1-\frac{i}{m}\right) r} P H E_{t-1+\frac{i}{m}}^{\psi},
$$

for any $t=1,2, \ldots, T-1$.

Then a new rate in $\underline{\theta_{t}}$ is determined for the following year such that the value of the EIA equals the accumulated value of the hedging portfolio plus the total amount of error incurred during that year. That is

$$
V_{t}^{C R}\left(\underline{\theta}_{t}\right)=V_{t^{-}}^{\psi}\left(\underline{\theta}_{t-1}\right)+Y H E_{t}^{\psi}
$$


The new rate in $\underline{\theta_{t}}$ will be the rate considered constant for all the subsequent years while pricing, and then the same steps are repeated each year. For example, consider a yearly CR EIA with a fixed participation rate $\alpha_{0}$, no spread, and a changing cap, that is, $\alpha_{t}=\alpha_{0}$ and $s_{t}=0$ for all $t$. First $\underline{\theta_{0}}=\left\{\alpha_{0}, c_{0}, 0\right\}$ is set such that $c_{0}$ satisfies (5.2.1). Then by the end of the first year, a new cap rate $c_{1}$ is determined such that $V_{1}^{C R}\left(\underline{\theta_{1}}\right)=V_{1^{-}}^{\psi}\left(\underline{\theta_{0}}\right)+Y H E_{1}^{\psi}$, where $\underline{\theta_{0}}=\left\{\alpha_{0}, c_{0}, 0\right\}$ and $\underline{\theta_{1}}=\left\{\underline{\alpha_{1}}, \underline{c_{1}}, 0\right\}$, with $\underline{c_{1}}=\left\{c_{0}, c_{1}\right\}$, and $\underline{\alpha_{1}}=\left\{\alpha_{0}, \alpha_{0}\right\}$. When the value at time $1, V_{1}^{C R}\left(\underline{\theta_{1}}\right)$, is calculated, $c_{0}$ is the cap rate applied for year 1 only, and the new rate $c_{1}$ is the cap rate assumed constant for the remaining years until maturity.

Following this method insures that the error is reset to zero at the beginning of each year, because the value of the EIA is changed - which is the amount owed by the issuer by exactly how much it costs him to hedge his position during each year. In other words, the issuer transfers the cost of hedging each year to the buyer by limiting his credited index-linked gain. Thus he protects himself against the additional cost incurred from the hedging strategy and maintains a "self-financing" over-all portfolio.

\subsubsection{Numerical Analysis}

It is logical to consider that issuers of such contracts will be, as much as buyers, interested in knowing the pattern of changes in these rates. Therefore, a detailed numerical analysis is conducted to replicate this pattern with the purpose of trying to eliminating the hedging errors.

A 7-year Annual Compound Ratchet EIA with the same set of default parameters introduced in Section 5.1.2 is analyzed. That is $T=7$, floor $=0, \mu=0.08, \sigma=0.2, r=$ 0.04 , and $m=52$ unless otherwise indicated. Assume a full participation rate in the index value guaranteed for the life of the contract, no spread, and a varying cap rate. Then the vector of parameters becomes $\underline{\theta_{t}}=\left\{\underline{\alpha_{t}}, \underline{c_{t}}, \underline{s_{t}}\right\}$, where $\alpha_{t}=100 \%$ and $s_{t}=0$ for all $t$. It follows that the value of the contract hereafter will only be written as a function of the 
variable vector $\underline{c}_{t}$. Even though the issuer retains the right to change the cap rate yearly, an initial rate $c_{0}$ has to be set at inception of the contract. This rate $c_{0}$ is calculated such that it is considered to remain constant until maturity.

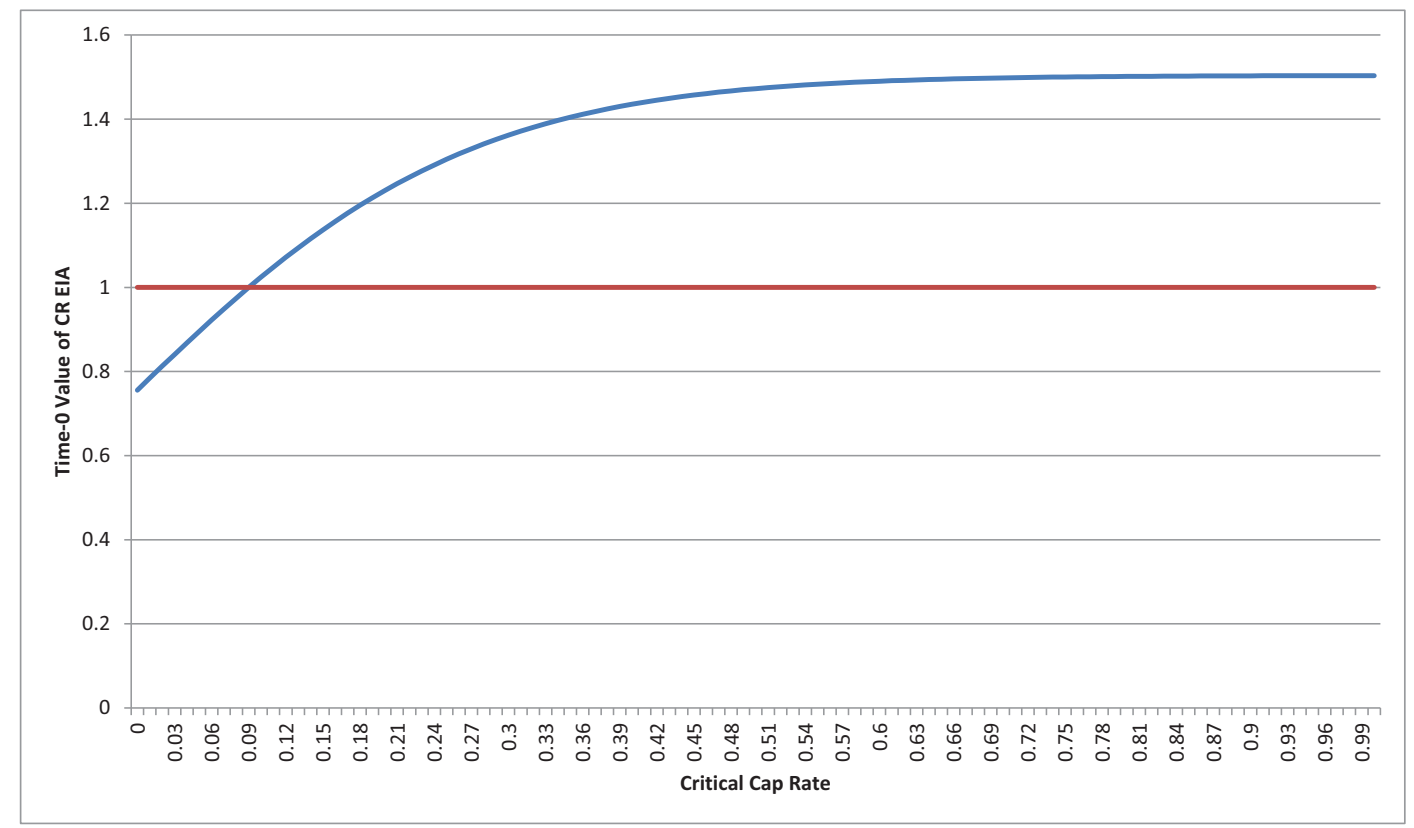

Figure 5.3: Time-0 value of an Annual Compound Ratchet EIA as a function of $c_{0}$.

Figure 5.3 traces the time-zero value of this CR EIA as a function of the cap rate initially considered constant for the whole term of the contract. The value increases monotonically as the local cap rate $c$ increases, while all other parameters are held constant. We assume that one can find a critical rate $c$ such that the time-0 value of the EIA is exactly equal to that of the index. In other words, all parameters are chosen such that there exists a critical rate that satisfies the following equation:

$$
V_{0}^{C R}\left(c_{0}\right)=1
$$


Therefore, the initial cap rate $c_{0}=9 \%$ is obtained numerically such that (5.2.4) is satisfied. Then the dynamic hedging strategy is applied and the hedging errors are extracted and recorded at the end of each period. Based on this information, at the end of each year $t=\{1,2, \ldots, T-1\}$, the value of the yearly hedging error $Y H E_{t}^{\psi}$ is calculated from (5.2.2). A new cap rate $c_{t}$, assumed constant for the remaining years until maturity, is obtained such that $V_{t}^{C R}\left(\underline{c}_{t-1}, c_{t}\right)=V_{t^{-}}^{\psi}\left(\underline{c}_{t-1}\right)+Y H E_{t}^{\psi}$ is satisfied. This new cap rate resets the issuer's yearly cost to zero by limiting the gain from the index return credited to the buyer. This algorithm is then repeated to get 25,000 simulations of the vector $\underline{c}_{T-1}=\left\{c_{0}, c_{1}, \ldots, c_{T-1}\right\}$, denoted by $\underline{c}$, and whose empirical distribution is to be analyzed as some of the parameters change.

\section{Gamma Hedging}

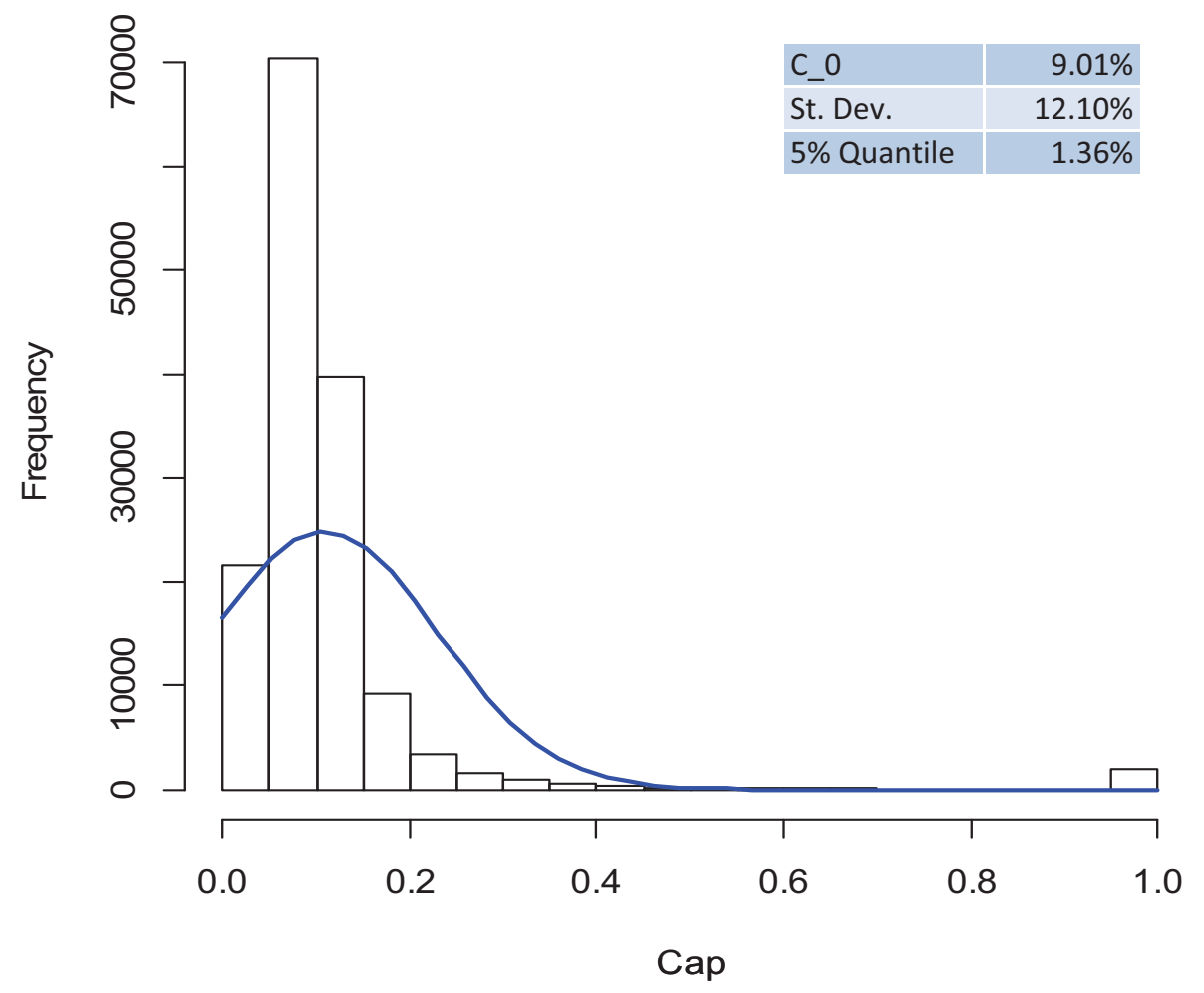

Figure 5.4: Histogram of $c$ at the default parameter set. 
Figure 5.4 represents the histogram of all yearly cap rates resulting from 25, 000 simulations of the vector $\underline{c}$, at the default parameter set and under the Gamma-hedging strategy. The blue curve represents the relative normal distribution curve. A quick analysis of this distribution shows a mean of $10.91 \%$ which is far from the critical rate $c_{0}$ by almost $1.9 \%$, and a significant standard deviation of $12.10 \%$. These figures show that this method can be indeed used to reproduce the changing cap rate over the term of the contract with a significant variation from the initial cap rate $c_{0}$.

\begin{tabular}{c||c|c|c|c|c|c|c|c|c|c} 
Quantiles & $1 \%$ & $2 \%$ & $3 \%$ & $4 \%$ & $5 \%$ & $6 \%$ & $7 \%$ & $8 \%$ & $9 \%$ & $10 \%$ \\
\hline \hline$c$ & 0.01 & 0.01 & 0.01 & 0.59 & 1.36 & 2.00 & 2.53 & 3.02 & 3.42 & 3.77
\end{tabular}

Table 5.2: Quantiles of the distribution of $c$, in percentages.

Additionally, it is possible to determine a minimum cap rate by examining the left tail of the empirical distribution of $\underline{c}$. Table 5.2 lists some values for the lower quantiles of $\underline{c}$. It is a difficult choice since, on the one hand, one can say $0.01 \%$ could be seen too low by the policyholder to be considered a minimum cap, on the other hand, a $3.7 \%$ minimum could be risky for the issuer and thus makes the contract too expensive. Therefore, we will consider the $5 \%$ quantile as a fair minimum cap rate for now, and use it to compare the results. For instance, the $5 \%$ quantile of the distribution is at $1.36 \%$, meaning that if this is the minimum value of local cap rates promised at inception, then there is a probability of $5 \%$ that a contract will over-estimate the pricing parameter, causing an un-covered loss for the issuer. Nevertheless, the impact of this choice on the hedging cost will be discussed in the last section.

\begin{tabular}{c||c|c|c|c|c}
$\mathrm{m}$ & 6 & 12 & 24 & 52 & 360 \\
\hline \hline St. Dev. & 13.81 & 13.13 & 12.52 & 12.10 & 11.91 \\
\hline Median & 8.76 & 8.87 & 8.95 & 8.98 & 9.01 \\
\hline 5\% Quantile & 0.37 & 0.83 & 1.19 & 1.36 & 1.49
\end{tabular}

Table 5.3: Effects of changing the number of trading dates $m$ (in percentages). 


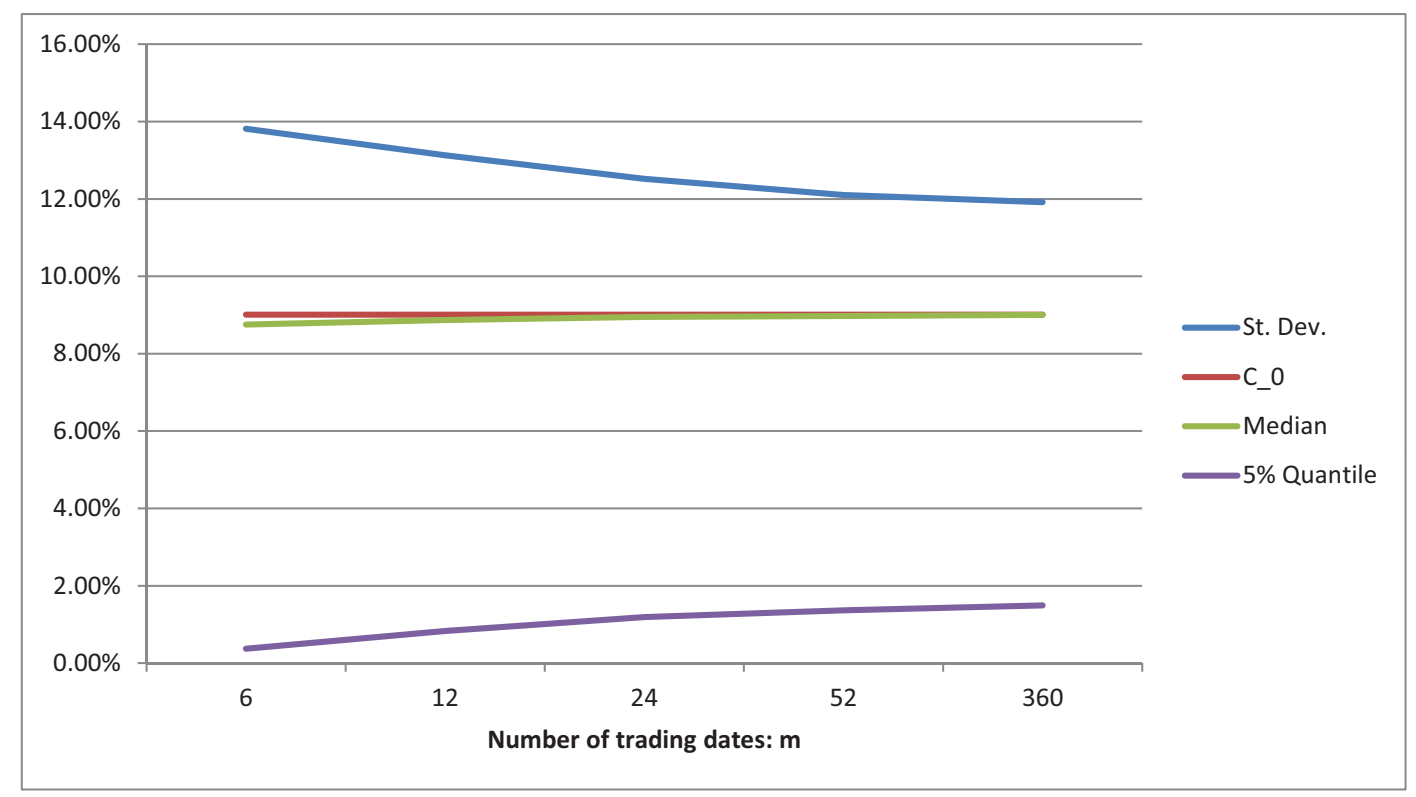

Figure 5.5: Effects of changing the number of trading dates $m$.

Another interesting thing to look at is what happens to the minimum cap rate as the issuer increases his hedging frequency. Theoretically, increasing the number the times the hedging portfolio is re-balanced per year - referred to by the number of trading dates $m$, reduces the impact of the model discretization. It follows that hedging errors are expected to be reduced, and thus yearly cap rates must not move far away from the critical rate $c_{0}$. This is exactly what can be concluded from Figure 5.5 with the corresponding values in Table 5.3. In fact, observe that as $m$ increases, the standard deviation is decreasing from $13.81 \%$ to $11.91 \%$. As a consequence of the less dispersion; the median converges to the critical rate to become exactly equal to $c_{0}=9.01 \%$ when trading daily, and the $5 \%$ quantile increases significantly from $0.37 \%$ to $1.49 \%$. That is, the more the issuer can re-balance his hedging portfolio, the more accurate his hedging is and the less hedging errors are incurred. Therefore, by bearing less risk, the issuer is able to promise the buyer higher minimum cap rate. 
However, even though it is converging, the $5 \%$ quantile of the hedging errors does not vanish, since even by increasing the trading frequency to daily, the $5 \%$ quantile of the empirical distribution is still far from $c_{0}$. That is, we can still observe a significant change in the cap rates from one year to another, and thus this new proposed hedging method will still be effective. However, as previously explained in Section 3.3.1, a trade-off between the hedging frequency and transaction costs must always be considered.

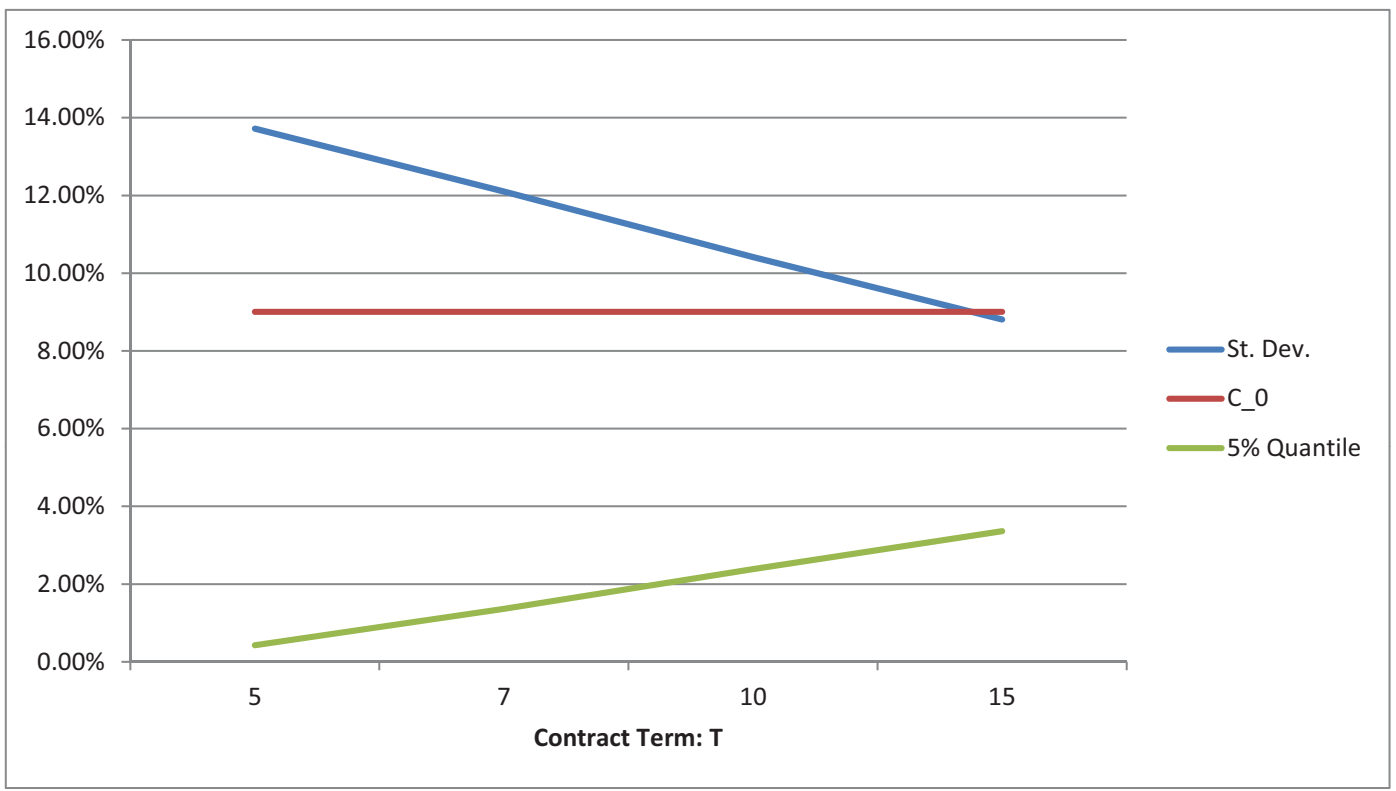

Figure 5.6: Effects of changing the contract term $T$.

\begin{tabular}{c||c|c|c|c}
$\mathrm{T}$ & 5 & 7 & 10 & 15 \\
\hline \hline St. Dev. & 13.72 & 12.10 & 10.42 & 8.81 \\
\hline 5\% Quantile & 0.43 & 1.36 & 2.39 & 3.36
\end{tabular}

Table 5.4: Effects of changing the contract term $T$ (in percentages).

One could also be interested in the effects of changing the term of the EIA contract on the cost of the hedging strategy and the pattern of the yearly cap rate, especially on 
the minimum promised or critical value. Figure 5.6 and Table 5.4 show a similar behavior to the one seen when changing the number of trading dates. The standard deviation decreases significantly from $13.72 \%$ to $8.81 \%$ followed by another significant increase in the $5 \%$ quantile of the distribution from $0.43 \%$ to $3.36 \%$. These results may seem surprising since one could logically think that increasing the contract term presents more uncertainty and thus more risk for the issuer of the EIA. Therefore it is expected that he should be more conservative in terms of lowering the minimum promised cap rate. However, the intuition is different. The increase in the minimum cap rate can be explained by the fact that the longer the term of the contract is, the less volatile the issuer's periodic cash flows are, and the more time he has to adjust for his losses. For that reason, he can tolerate allowing for higher index returns to be credited to the buyer by increasing the minimum cap rate as the contract term increases. This is a very interesting result since it will help the issuer retain his policyholders for longer periods by promising higher returns.

\begin{tabular}{c||c|c|c|c}
$f$ & $0 \%$ & $0.25 \%$ & $0.5 \%$ & $1 \%$ \\
\hline \hline$c_{0}$ & 9.01 & 8.70 & 8.39 & 7.77 \\
\hline $5 \%$ Quantile & 1.36 & 1.12 & 0.92 & 0.45
\end{tabular}

Table 5.5: Effects of changing the floor rate $f$ (in percentages).

Table 5.5 shows the impact of changing the floor rate $f$ on the cap rate. As the cap rate imposes a ceiling on the credited returns, the floor rate provides a protection for the buyer by promising a minimum return to be credited. Therefore, both rates if increased will increase the value of the EIA. This explain their impact on each other. That is, the increase in $f$ from 0 to $0.5 \%$ is offset by a decrease in $c_{0}$ from $9.01 \%$ to $8.39 \%$. Which also results in decreasing the minimum cap rate from $1.36 \%$ to $0.92 \%$. The latter behavior can be explained by the fact that the higher minimum credited return is promised, the more risk the issuer has to bear to meet his liabilities in case the index performs badly, therefore, the more conservative he should be by lowering the ceiling on the returns. That is, in a bull market, he benefits from the additional gain that is not credited to the buyer to offset the loss from crediting high returns in a bear market. Yet, observe that in the 
case where the floor rate is set to $1 \%$, the minimum cap goes as low as $0.45 \%$, even lower than the floor. This says that a floor of $1 \%$ is too high to promise, given that the issuer wishes to be protected $95 \%$ of the time. In this case, a quantile higher than the $5 \%$ should be considered in order to offer a reasonable minimum cap rate.

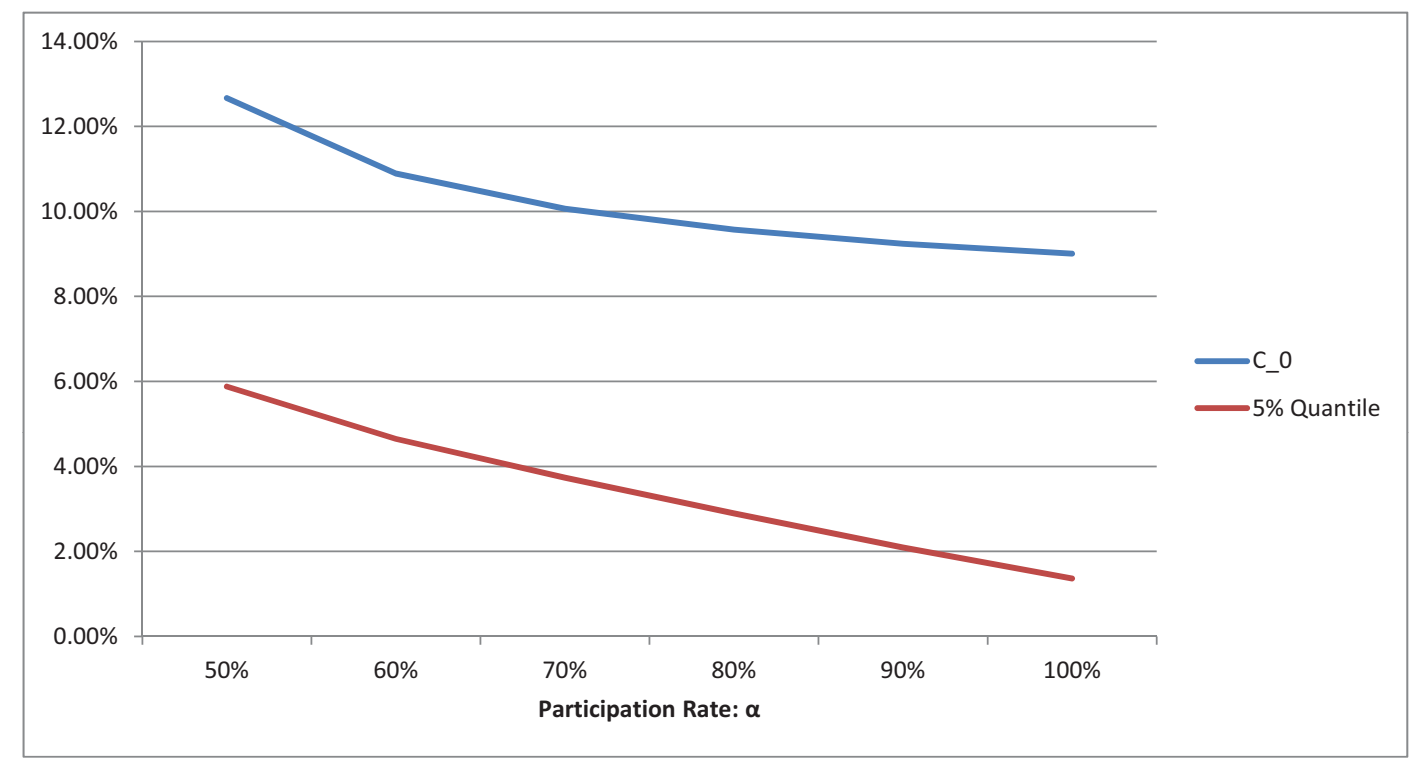

Figure 5.7: Effects of changing the participation rate $\alpha$.

Figure 5.7 shows the effect of changing the participation rate $\alpha$ on the critical cap rate $c_{0}$ and the minimum cap rate represented by the $5 \%$ quantile of the empirical distribution. As the figure shows, increasing the participation rate from $50 \%$ to $100 \%$ leads to a decrease in the critical cap rate from $12.67 \%$ to $9.01 \%$. Similarly, the minimum cap rate decreases from $5.88 \%$ to $1.36 \%$. This result is expected since these two parameters have an opposite impact on the price of the EIA contract. That is, increasing the participation rate allows for more gain to be credited to the buyer, whereas increasing the cap rate limits the amount of gain from the index to be credited as return. 


\begin{tabular}{c||c|c|c|c|c|c}
$\alpha$ & $50 \%$ & $60 \%$ & $70 \%$ & $80 \%$ & $90 \%$ & $100 \%$ \\
\hline \hline$c_{0}$ & 12.67 & 10.89 & 10.06 & 9.57 & 9.24 & 9.01 \\
\hline $\mathrm{d}_{c_{0}}$ & - & -1.77 & -0.83 & -0.49 & -0.33 & -0.23 \\
\hline \hline $5 \%$ Quantile & 5.88 & 4.65 & 3.74 & 2.89 & 2.09 & 1.36 \\
\hline $\mathrm{d}_{Q u .}$ & - & -1.23 & -0.91 & -0.84 & -0.80 & -0.73
\end{tabular}

Table 5.6: Effects of changing the participation rate $\alpha$ (in percentages).

Furthermore, Table 5.6 shows the changes in the values of $c_{0}$, denoted by $d_{c_{0}}$, as well as the changes in the values of the $5 \%$ quantiles, denoted by $d_{Q u}$., for some increasing values of $\alpha$. For example, the first value in $d_{c_{0}}$ represents the magnitude of decrement in $c_{0}$ as $\alpha$ goes from $50 \%$ to $60 \%$. These results show that, as $\alpha$ increases steadily by $10 \%$, $d_{c_{0}}$ and $d_{Q u}$. decrease at a faster rate. That is, as $\alpha$ increases by $10 \%$ from $50 \%$ to $60 \%$, the magnitude of the decrement in $c_{0}$ is $1.77 \%$, whereas for another $10 \%$ increase in $\alpha$ from $90 \%$ to $100 \%$, the magnitude of the decrement in $c_{0}$ decreases to $0.23 \%$. This means that the value of the contract is more sensitive to changes in the cap rates than changes in the participation rates, additionally, it becomes more sensitive to smaller changes in the cap rates for higher values of $\alpha,(\alpha \geq 80 \%)$.

\begin{tabular}{c||c||c|c|c|c}
$5 \% \mathrm{Qu}$. & $\alpha$ & $40 \%$ & $50 \%$ & $60 \%$ & $70 \%$ \\
\hline \hline \multirow{2}{*}{$\sigma_{1}=20 \%$} & $r_{1}=3 \%$ & 4.59 & 3.37 & 2.40 & 1.60 \\
& $r_{2}=4 \%$ & 9.43 & 5.88 & 4.65 & 3.74 \\
\hline \hline \multirow{2}{*}{$\sigma_{2}=30 \%$} & $r_{1}=3 \%$ & 2.56 & 1.27 & 0.11 & 0.01 \\
& $r_{2}=4 \%$ & 4.98 & 3.54 & 2.31 & 1.19
\end{tabular}

Table 5.7: Values of the 5\% quantile (in percentages) for two values of $\sigma$ and $r$ as $\alpha$ increases.

Table 5.7 shows the values of the $5 \%$ quantile of the distribution of $\underline{c}$ as $\alpha$ increases steadily by $10 \%$, for each of the four cases; a low volatility market $\sigma_{1}=20 \%$ or a high volatility market $\sigma_{2}=30 \%$ with either $r_{1}=3 \%$ or $r_{2}=4 \%$. From this table, Tables 5.8 , 5.9 and 5.10 are created to analyze different scenarios separately.

On the one hand, Table 5.7 shows that increasing the risk-free rate of return $r$ leads to 


\begin{tabular}{c||c|c|c|c|c} 
& $\alpha$ & $40 \%$ & $50 \%$ & $60 \%$ & $70 \%$ \\
\hline \hline$\sigma_{1}=20 \%$ & \multirow{2}{*}{$r_{2}-r_{1}$} & 4.85 & 2.51 & 2.24 & 2.13 \\
$\sigma_{2}=30 \%$ & & 2.42 & 2.27 & 2.19 & 1.18
\end{tabular}

Table 5.8: Magnitude of increment in the 5\% quantile of $c$ as $r$ increases from $3 \%$ to $4 \%$.

higher minimum cap rates, which makes sense since higher risk-free returns decrease the value of EIA therefore, the issuer has to increase the value by raising the ceiling. However, observe from Table 5.8 that this increment is less significant as sigma is increased. This means that in high volatile markets, the minimum cap rates become less sensitive to changes in $r$.

\begin{tabular}{c||c|c|c|c|c} 
& $\alpha$ & $40 \%$ & $50 \%$ & $60 \%$ & $70 \%$ \\
\hline \hline$r_{1}=3 \%$ & \multirow{2}{*}{$\sigma_{2}-\sigma_{1}$} & -2.03 & -2.09 & -2.29 & -1.60 \\
$r_{2}=4 \%$ & & -4.46 & -2.34 & -2.34 & -2.55
\end{tabular}

Table 5.9: Magnitude of decrement in the 5\% quantile of $c$ as $\sigma$ increases from $20 \%$ to $30 \%$.

On the other hand, Table 5.7 shows that increasing the market volatility leads to lower minimum cap rates, regardless of the value of $\alpha$ or $r$. Which also makes sense since the more risk the issuer has to bear the more conservative he should be and therefore lowering the ceiling on the credited returns. Nevertheless, this decrement is more significant as the risk-free rate of return $r$ also increases form $3 \%$ to $4 \%$. This can be explained by the fact that the impact of the volatility on the cap rates is higher than that of $r$, its counter-effect on $c$ diminishes even more as $\sigma$ increases.

\begin{tabular}{l||c|c||l} 
& $\alpha=40 \%$ & $\alpha=50 \%$ & $\begin{array}{l}\text { magnitude of } \\
\text { decrement }\end{array}$ \\
\hline \hline$\sigma=20 \%$ & 9.43 & 5.88 & 3.55 \\
$\sigma=30 \%$ & 4.98 & 3.54 & 1.44
\end{tabular}

\begin{tabular}{c|c||l}
$\alpha=60 \%$ & $\alpha=70 \%$ & $\begin{array}{l}\text { magnitude of } \\
\text { decrement }\end{array}$ \\
\hline \hline 4.65 & 3.74 & 0.91 \\
2.31 & 1.19 & 1.12
\end{tabular}

Table 5.10: Values of the 5\% quantile (in percentages) showing the effect of $\alpha$ in low and high volatility markets. 
Additionally, as expected, the minimum cap rate decreases as $\alpha$ increases, in any of the four cases. Observe from Table 5.10 that the magnitude of decrement in high volatility markets (1.44) is less than the magnitude of decrement in low volatility markets (3.55) as $\alpha$ goes from $40 \%$ to $50 \%$, however it is more (1.12 in high vol and 0.91 in low vol) as $\alpha$ goes from $60 \%$ to $70 \%$. This can be explained by the fact that increasing $\alpha$ boosts the effect of the volatility. Therefore the decrease in the minimum cap rate becomes more significant in high volatile markets than in low volatile markets for high ranges of $\alpha$. 


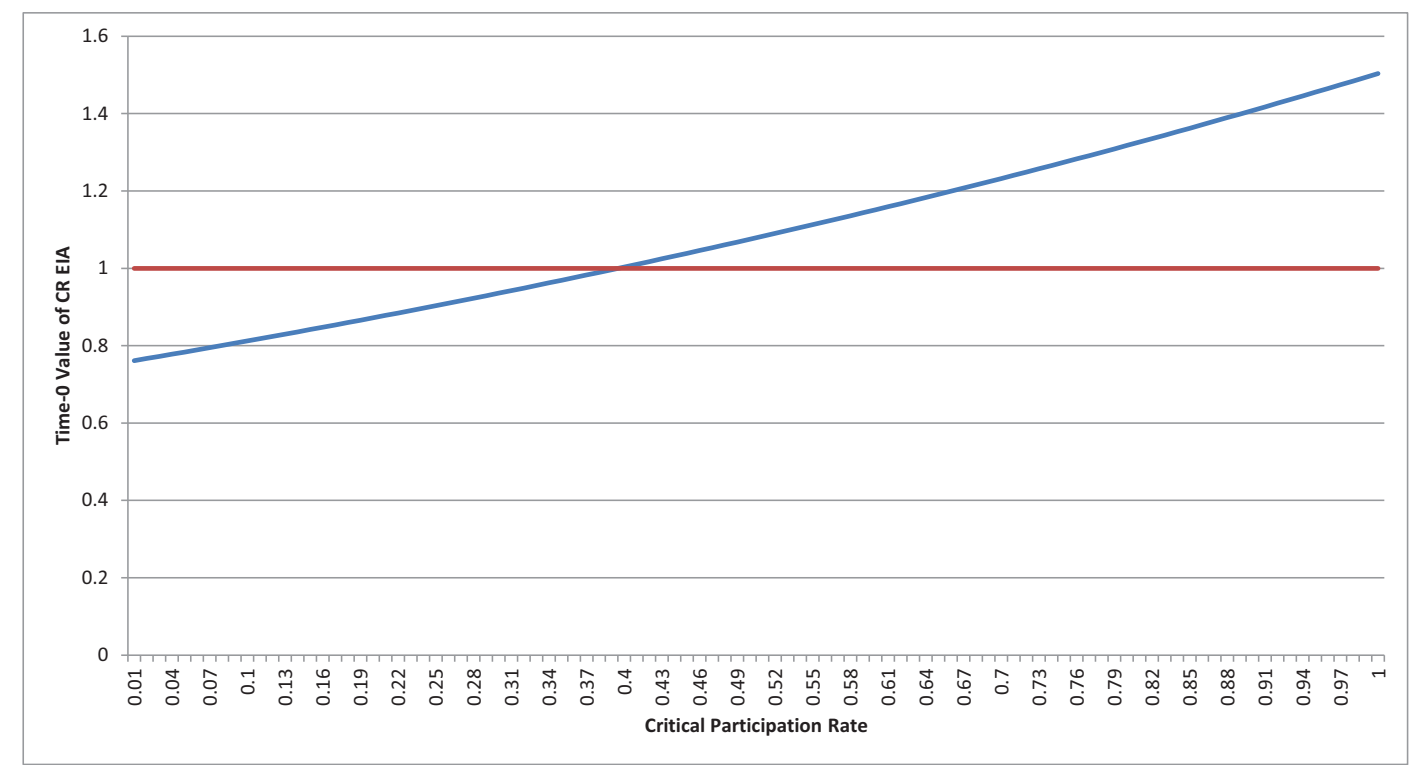

Figure 5.8: Time-0 value of an Annual Compound Ratchet EIA as a function of $\alpha_{0}$.

A similar analysis can be done with the participation rate $\alpha$ being the changing pricing parameter and the rest assumed constant over the contract term. In this case we have $c_{t}=c_{0}$ and $s_{t}=s_{0}$ for all $t$, and $\underline{\alpha}_{T-1}=\left\{\alpha_{0}, \alpha_{1}, \ldots, \alpha_{T-1}\right\}$. Figure 5.8 traces the time- 0 value of this CR EIA with as a function of the participation rate initially considered constant for the whole term of the contract. Similarly to Figure 5.3, the time-0 price increases monotonically as the participation rate increases, while all other parameters are held constant, and a critical rate $\alpha_{0}=39.51 \%$ is solved numerically such that the price of the EIA is exactly equal to that of the underlying index by satisfying $V_{0}^{C R}\left(\alpha_{0}\right)=1$. Then the Gamma-hedging strategy along with the proposed method are applied to get 25,000 of the vector $\underline{\alpha}_{T-1}$.

The analyses of $\underline{\alpha}$ is very similar to that of $\underline{c}$. For example, Figure 5.9 represents the empirical distribution of the yearly participation rates resulting from 25, 000 simulations of the vector $\underline{\alpha}$, under the Gamma-hedging strategy. Observe that the median converges 


\section{Gamma Hedging}

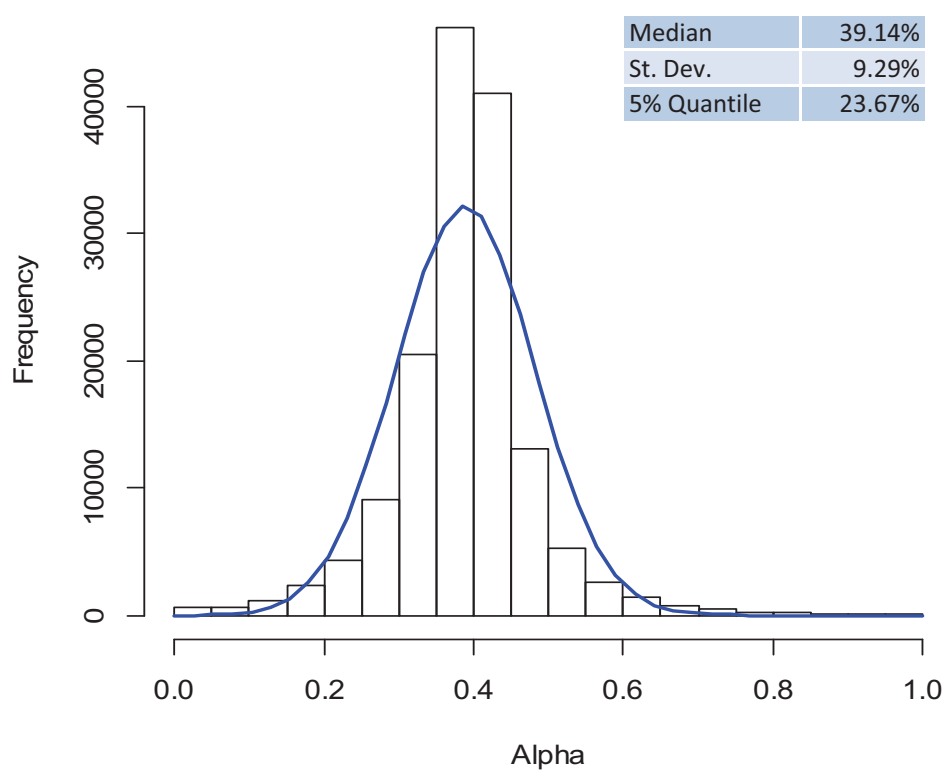

\section{Gamma Hedging}

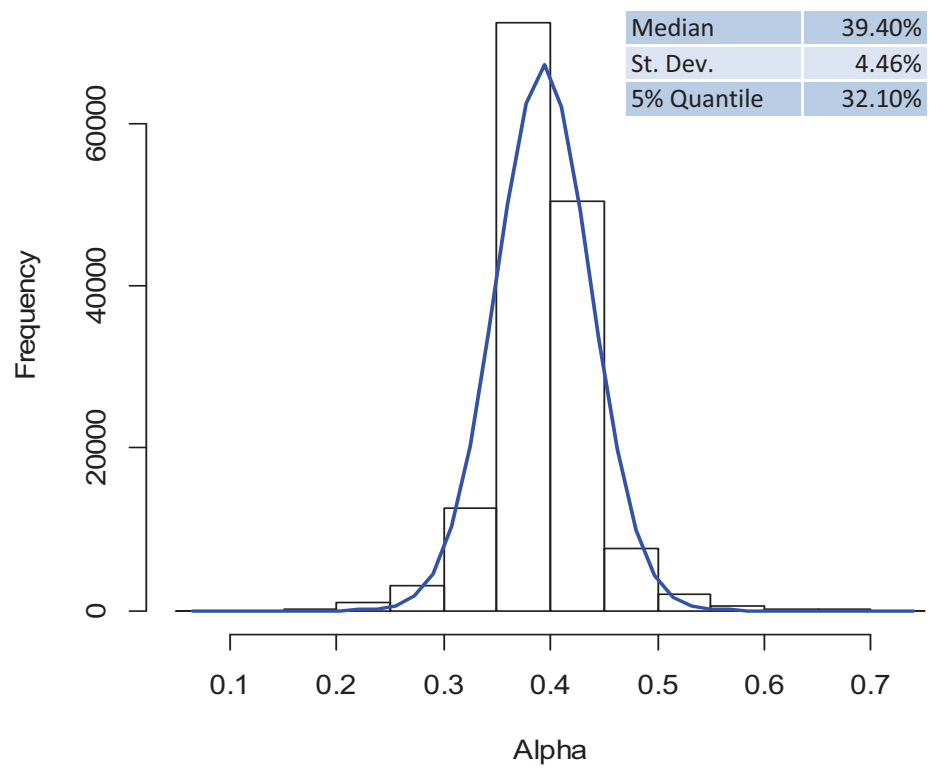

Figure 5.9: Histograms comparing the distribution of $\alpha$ as the number of trading dates per year increases. 
to $\alpha_{0}$, the standard deviation decreases by $4.83 \%$ and the $5 \%$ quantile of the empirical distribution increases, all to conclude again that the issuer is able to promise the buyer a higher minimum participation rate as he re-balances his hedging portfolio more frequently, with only $5 \%$ chance of over-estimating the participation rate.

\begin{tabular}{c||c|c|c|c|c|c|c|c|c|c} 
Quantiles & $1 \%$ & $2 \%$ & $3 \%$ & $4 \%$ & $5 \%$ & $6 \%$ & $7 \%$ & $8 \%$ & $9 \%$ & $10 \%$ \\
\hline \hline$\underline{\alpha}$ & 25.97 & 28.63 & 30.23 & 31.29 & 32.10 & 32.80 & 33.36 & 33.85 & 34.25 & 34.60
\end{tabular}

Table 5.11: Quantiles of the distribution of $\alpha$, in percentages.

Additionally, Table 5.11 lists the lower quantiles of the empirical distribution of $\underline{\alpha}$ at the default parameter set.

\subsubsection{Hedging Errors After Applying the Proposed Strategy}

Recall from (5.2.2) that the last yearly hedging error that could be reset to zero is $Y H E_{T-1}$, since the last new rate $\underline{\theta}_{T-1}$ to be applied during year $T$, is found such that (5.2.3) at $t=T-1$ is satisfied.

Therefore, following this strategy, the issuer would be able to transfer almost all yearly hedging errors to the buyer by resetting the chosen pricing parameter each year, except for the hedging error during the last year $Y H E_{T}$ which he has to bear, and would then be considered the cost of his hedge. That is,

$$
Y H E_{T}^{\psi}=\sum_{i=1}^{m} e^{\left(1-\frac{i}{m}\right) r} P H E_{T-1+\frac{i}{m}}^{\psi}
$$

where $P H E_{t}^{\psi}$ is given by

$$
P H E_{t}^{\psi}=V_{t^{-}}^{\psi}-V_{t}^{C R} .
$$

Then the cost of hedge would be the present value at time-zero of last year's hedging 
errors;

$$
\mathrm{COH}^{\psi}=e^{-T r} Y H E_{T}^{\psi}
$$

Additionally, the issuer has to promise the buyer a minimum pricing parameter, predetermined at inception. Then, as the new pricing parameter is calculated every year, whenever is it found to be less than the minimum, the actual credited parameter during that year will be the minimum. In this case, the value of the CR EIA will be higher than that of the issuer's hedging portfolio, and thus, the issuer has to bear the risk of allowing for more return to credited than what his hedging strategy allows him to. Therefore, a hedging error will arise during that year and will be carried on to the following year. If the second year's new pricing parameter does not correct it, it will keep on being carried on until it is added to the last year's error, to constitute the total hedging errors, which is then discounted back to time zero to make up the final cost.

Figure 5.10 shows the hedging cost after applying the proposed method with a Gammahedging strategy, if the $5 \%$ quantile of the empirical distribution is considered to be the minimum rate for the chosen pricing parameter $\alpha$. The hedging cost is significantly reduced, with the mean decreasing from $-0.13 \%$ to $-0.021 \%$, the standard deviation from $1.93 \%$ to $0.769 \%$, as well as the $1 \%$ quantile from $-4.98 \%$ to $-2.058 \%$, compared to the hedging cost using only the Gamma-hedging strategy as shown in Figure 5.2.

\begin{tabular}{c||c|c|c} 
Minimum $\alpha$ & Mean & St. Dev. & $1 \%$ Quantile \\
\hline \hline $1 \%$ Quantile $=25.97 \%$ & -0.018 & 0.764 & -2.041 \\
\hline $5 \%$ Quantile $=32.10 \%$ & -0.021 & 0.769 & -2.058 \\
\hline $10 \%$ Quantile $=34.60 \%$ & -0.021 & 0.788 & -2.091
\end{tabular}

Table 5.12: Mean, standard deviation, and 1\% quantile of the final hedging cost for different choices of minimum rates.

Table 5.12 compares the values of the mean, standard deviation, and $1 \%$ quantile of the hedging cost after applying the proposed method with a Gamma-hedging strategy for each of the $1 \%, 5 \%$, and $10 \%$ quantiles considered as the minimum participation rate. 


\section{Gamma Hedging}

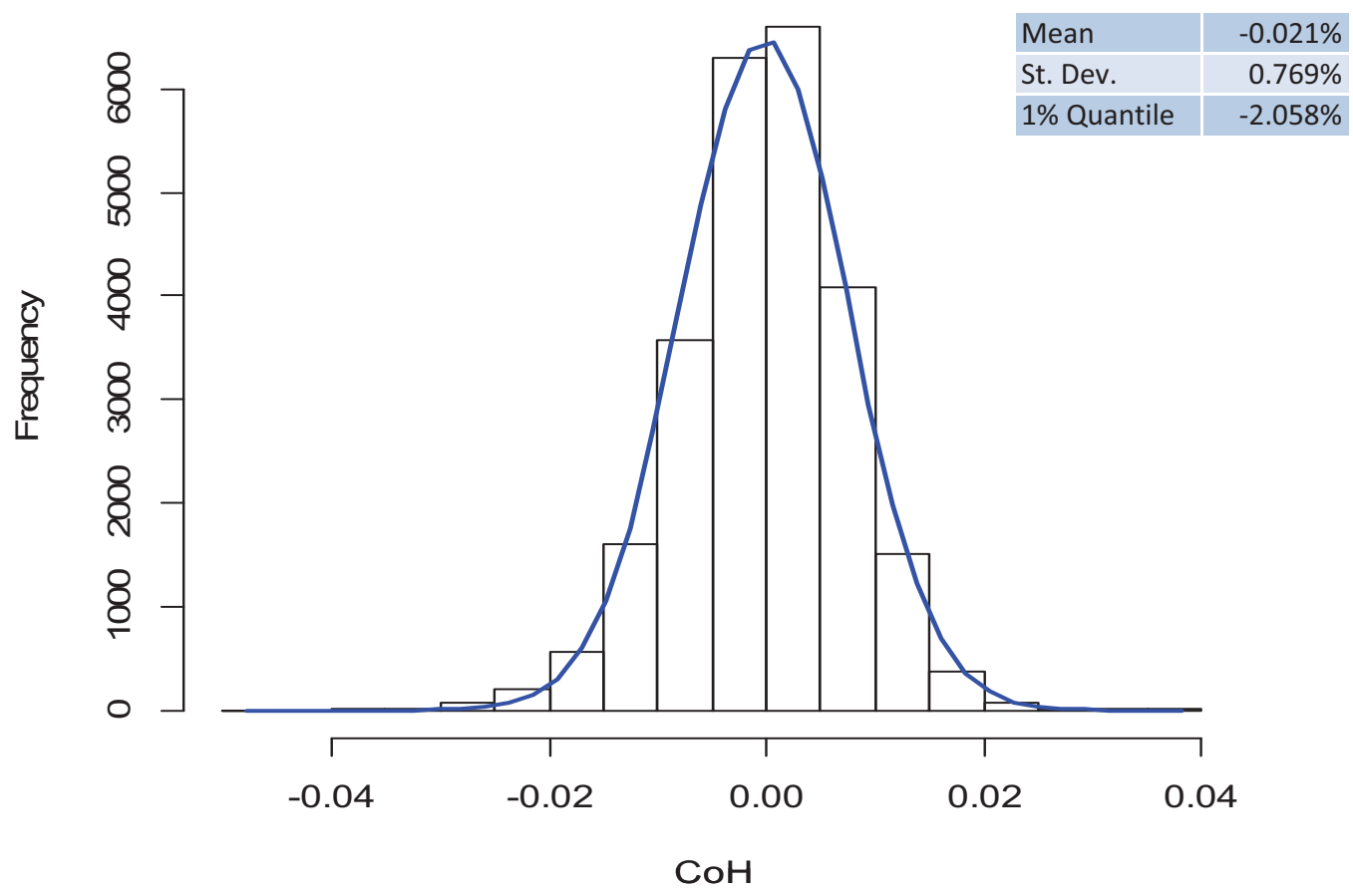

Figure 5.10: Cost of hedge after applying the proposed method, if the $5 \%$ quantile is considered as the minimum rate.

Observe that, as the minimum participation rate decreases, the reductions in the values are very small. For example, the standard deviation of errors is only reduced by $0.019 \%$ and $0.005 \%$ as the minimum rate decreases from the $10 \%$ to the $5 \%$ then to the $1 \%$ quantile. It follows that, if the issuer find these differences to be insignificant, then being more conservative is not a good idea, instead, he will probably be better off promising higher returns by increasing the minimum and thus attracting more buyers. 


\section{Conclusion}

It is expected that EIAs will continue to experience a prolonged period of rapid growth, as they offer participation in the equity market upturns while keeping the policyholder protected from the downside risk. The main purpose of this thesis was to propose and test a new approach to hedge CR EIAs and simultaneously protect the issuer, as much as possible, from hedging risk based on changing the pricing parameters.

After finding closed-form solutions for the value of a CR EIA at any time throughout the contract term, dynamic hedging strategies using the Greeks are presented and implemented in a numerical example. Their efficiency is analyzed through extracting the hedging errors resulting from the discretization process. The numerical analysis shows that the Gamma-hedging strategy improves the performance of the Delta-hedging strategy by reducing the magnitude of the hedging errors, and thus lowering the cost of the hedge for the issuer.

The performance of the Gamma-hedging strategy is further improved by applying the proposed approach of transferring the errors from the issuer to the policyholder (and vice-versa) through changing the pricing parameter. A detailed numerical analysis is implemented to extract the distribution of the pricing parameters. A minimum rate is chosen at the $5 \%$ quantile of the empirical distribution with the purpose of examining its behavior as some of the initial parameters change. For instance, by increasing the hedging frequency, the issuer is able to promise the policyholder a higher minimum cap rate. Nevertheless, an interesting result is that no matter how much the trading frequency 
is increased, the minimum rate does not vanish, which shows that our method is effective. However, a trade-off between the hedging frequency and transaction costs must always be considered. Another interesting result is seen when increasing the contract term results in an increase in the minimum rate, which implies that issuers are able to retain their policyholders for longer periods by promising higher returns.

Finally, since the issuer has to promise a minimum pricing parameter at inception, there would still be a portion of hedging errors uncovered and retained by the issuer, in addition to the last year's errors only. These make up the new hedging cost for the issuer which is shown to be significantly reduced, as compared to the cost from using only the Gamma-hedging strategy. Moreover, the choice of using the minimum rate is revisited by comparing the final hedging cost with the $10 \%$ quantile to the cost with the $5 \%$ and to that with the $1 \%$ quantile. Interestingly, one can conclude that the reduction in the cost from being much more conservative is not very significant, instead, the issuer is probably better off promising higher returns by increasing the minimum rate and by that attracting more policyholders.

Future research could repeat the same work with other, more complicated, financial models. The reason behind choosing Black-Scholes was because it yields closed-form solutions for the valuation of CR EIAs, thus reducing the need for estimations, and providing a clear way to introduce this new idea and test that it is working successfully. To extend this work, it could be interesting to include transaction costs as well as stochastic volatilities and interest rates. Moreover, the addition of mortality and surrender risk should be explored. 


\section{Bibliography}

L. Bachelier. Théorie de la spéculation, Annales de l'Ecole Normale Supérieure, volume 3. Gauthier-Villars, Paris, 1900.

A. Bacinello, E. Biffis, and P. Millossovich. Variable annuities, a unifying valuation approach. Journal of Insurance, Mathematics and Economics, 49:285-297, 2011.

D. Bauer, A. Kling, and J. Russ. A universal pricing framework for guaranteed minimum benefits in variable annuities. ASTIN Bulletin, 38:621-651, 2008.

C. Bernard and P. Boyle. Natural hedge of volatility risk in equity indexed annuities. Annals of Actuarial Science, 5, 2011.

F. Black and M. Scholes. The pricing of options and corporate liabilities. The Journal of Political Economy, 81(3):637-654, 1973.

P. Boyle and E. Schwartz. Equilibrium prices of equity-linked insurance policies with an asset value guarantee. Journal of Risk and Insurance, 44:639-660, 1977.

P. Boyle, Y. Lai, and K. Tan. Using lattice rules to value low dimensional derivative contracts. Working paper, University of Waterloo, 2001.

M. Brennan and E. Schwartz. The pricing of equity-linked life insurance policies wth an asset value guarantee. Journal of Financial Economical, 3:195-213, 1976.

D. Brigo and F. Mercurio. Interest Rate Models, Theory and Practice. Springer Berlin Heidelberg, New York, 2007. 
Z. Chen, K. Vetzal, and P. Forsyth. The effect of modelling parameters on the value of gmwb guarantees. Journal of Insurance, Mathematics and Economics, 43:165-173, 2008.

L. Clewlow and S. Hodges. Optimal delta hedging under transactions costs. The Journal of Economic Dynamics and Control, 21:1353-1376, 1997.

L. Ederington. The hedging performance of the new futures markets. The Journal of Finance, 34:157-170, 1979.

E. Fama. Efficient capital markets: A review of theory and empirical work. The Journal of Finance, 25:383-417, 1969.

H. Follmer and M. Schweizer. Hedging by sequential regression, an introduction to the mathematics of option trading. Astin Bulletin, 18:147-160, 1988.

P. Gaillardetz and J. Lakhmiri. A new premium principle for equity indexed annuities. The Journal of Risk and Insurance, 78:245-265, 2011.

P. Gaillardetz and X. Lin. Valuation of equity linked insurance and annuity products with binomial models. North American Actuarial Journal, 10:117-144, 2006.

H. Gerber and E. Shiu. Option pricing by esscher transforms. Transactions of the Society of Actuaries, 46:99-191, 1994.

F. Godin, G. Gauthier, and P. Francois. Optimal hedging when the underlying asset follows a regime switching markov process. European Journal of Operational Research, $237: 312-322,2014$.

S. Haberman and G. Piscopo. Mortality risk and the valuation of annuities with guaranteed minimum death benefit options, application to the italian population. Actuarial Research Paper No. 187, Cass Business School, 68, 2008.

S. Haberman and G. Piscopo. The valuation of guaranteed lifelong withdrawal benefit options in variable annuity contracts and the impact of morttality risk. The North American Actuarial Journal, 15:59-76, 2011. 
M. Hardy. Investment Guarantees, Modeling and Risk Management for Equity linked Insurance Life Insurance. Wiley, New York, 2003.

M. Hardy. Ratchet equity indexed annuities. In 14th Annual International AFIR Colloquium, 2004.

M. Hardy and J. Wirch. The iterated cte. North American Actuarial Journal, 8:62-75, 2004.

J. Harrison and S. Pliska. Martingales and stochastic integrals in the theory of continuous trading. Stochastic Processes and Their Applications, 10:215-260, 1981.

R. Heynen and H. Kat. Selective memory. Journal of Risk, 7:73-76, 1994.

M. Hsieh and Y. Chiu. Monte carlo methods for valuation of ratchet equity indexed annuities. In proceedings of the 2007 Winter Simulation Conference, ed. S. Henderson, B. Biller, M.H. Hsieh, and J. Shortle. New Jersey USA Insitute of Electrical and Electronics Engineers, Inc., pages 998-1003, 2007.

S. Jaimungal. Pricing and hedging equity indexed annuities with variance gamma deviates. Working Paper, Department of Statistics, University of Toronto, 2004.

N. El Karoui and N. Quenez. Dynamic programming and pricing of contingent claims in an incmplete market. SIAM Journal on Control and Optimization, 33:29-66, 1995.

M. Kijima and T. Wong. Pricing of ratchet equity indexed annuities under stochastic interest rates. Journal of Insurance, Mathematics and Economics, 41:317-338, 2007.

M. Krayzler, R. Zagst, and B. Brunner. Closed-form solutions for guaranteed minimum accumulation benefits. Technische Universitat Munchen, Germany, 2011.

Y-K. Kwok. Mathematical Models of Financial Derivatives. Springer, Berlin Heidelberg, 2008.

T. L. Lai and T. W. Lim. Option hedging theory under transaction costs. The Journal of Economic Dynamics and Control, 33:1945-1961, 2009. 
H. Lee. Pricing equity-indexed annuities with path-dependent options. Journal of Insurance, Mathematics and Economics, 33:677-690, 2003.

LIMRA. U.s. individual annuity sales survey, (fourth quarter 2015). LIMRA Secure Retirement Institute, 2015.

C. Lin. Valuation of equity indexed annuities under regime switching jump model, evidence from stock indices (master's thesis). 2010.

X. Lin, K. Tan, and H. Yang. Pricing annuity guarantees under a regime switching model. The North American Actuarial Journal, 13:316-332, 2009.

X. Sheldon Lin and K. Tan. Valuation of equity-indexed annuities under stochastic interest rates. North American Actuarial Journal, 7:72-91, 2003.

A. MacKay. Pricing and hedging equity linked products under stochastic volatility models. (Master's Thesis) Concordia University, Montreal, QC, 2011.

B. Malkiel. The efficient market hypothesis and its critics. Journal of Economic Perspectives, 17:59-82, 2003.

J. Marrion. Advantage index product third quarter 2005 sales report. part 1. Advantage Compendium, Ltd.:Marylad Heights, MO, 2005.

G. Marshall, M. Hardy, and D. Saunders. Valuation of guaranteed minimum income benefit. North American Actuarial Journal, 14:38-58, 2010.

McKinsey and Company. Responding to the variable annuity crisis. The McKinsey Quarterly, New York, 2009.

M. Milevsky and S. Posner. The titanic option, valuation of the guaranteed minimum death benefit in variable annuities and mutual funds. The Journal of Risk and Insurance, 68:93-128, 2001.

M. Moghtadai. Partial hedging of equity linked products in the presence of policyholder surrender using risk measures (master's thesis). 2014. 
T. Moller. Risk-minimizing hedging strategies for unit linked life insurance contratcs. ASTIN Bulletin, 28:17-47, 1998.

T. Moller. Risk-minimizing hedging strategies for insurance payment proceses. Journal of Finance and Stochastics, 5:419-446, 2001a.

T. Moller. Hedging equity linked life insurance contratcs. North American Actuarial Journal, 5:79-95, 2001b.

M. Monoyios. Option pricing with transaction costs using a markov chain approximation. The Journal of Economic Dynamics and Control, 28:889-913, 2004.

L. Nielsen. Pricing and Hedging of Derivative Securities. Oxford University Press Inc., New York, USA, 1999.

L. Quian, W. Wang, R. Wang, and Y. Tang. Valuation of equity indexed annuity under stochastic mortality and interest rates. Journal of Insurance, Mathematics and Economics, 47:123-129, 2010.

P. Samuelson. Rational theory of warrant pricing. Industrial Management Review, 6: 13-32, 1965.

M. Schulmerich. Real Options Valuation, The Importance of Interest Rate Modelling in Theory and Practice. Springer Heidelberg Dordrecht, London New York, 2010.

M. Schweizer. Variance optimal hedging in discrete time. Mathematics of Operations Research, 20:1-32, 1995.

W. Sloane. Life insurers, variable annuities and mutual funds, a critical study. Journal of Risk and Insurance, 37:99, 1970.

E. Thorp and S. Kassouf. Beat the Market, A Scientific Stock Market System. Random House, New York, 1967.

S. Tiong. Valuing equity indexed annuities. North American Actuarial Journal, 4:149-170, 2001. 
N. Wiener. Differential space. Journal of Mathematical Physics 2, 2:131-174, 1923.

P. Wilmott. Paul Wilmott Introduces Quantitative Finance. John Wiley and Sons, Ltd, England, 2007.

M. Xu. Risk measure pricing and hedging in incomplete markets. Annals of Finance, 2: $51-71,2006$.

V. Zakamouline. European option pricing and hedging with both fixed and proportional transaction costs. The Journal of Economic Dynamics and Control, 30:1-25, 2006. 